\title{
The Computation of
}

\section{Resistive MHD Instabilities}

\section{In Axisymmetric Toroidal Plasmas}

\section{T. R. Harley ${ }^{+}$, C. Z. Cheng, S. C. Jardin}

\author{
Princeton University \\ Plasma Physics Laboratory \\ P. O. Box 451 \\ Princeton, NJ 08543
}

\begin{abstract}
We describe the linear MHD eigenmode code NOVA-R, which calculates the resistive stability of axisymmetric toroidal equilibria. A formulation has been adopted which accurately resolves the continuum spectrum of the ideal MHD operator. The resistive MHD slability equations are transformed into three coupled second order equations, one of which recovers the equation solved by the NOVA code in the ideal limit [1?: The eigenfunctions are represented by a Fourier expansion and cubic B-spline finite elements which are packed about the internal boundary layer. Accurate results are presented for dimensionless resistivitjes as low as $10^{-30}$ in cylindrical geometry. Fur axisymmet ric toroidal plasmas we demonstrate the accuracy of the NOSA-R cade by recovering ideal results in the $\eta \rightarrow 0$ limis, and cylirdrical resistive interchange results in the $a / R \rightarrow 0$ limit. $J^{\prime}$ analysis performed using the eigenfunctions computed by the $\mathrm{NOVA}-\mathrm{R}$ code agree with the asymptotic matching results frorn the resistive PEST 2 code for zero beta equilibria.
\end{abstract}

\footnotetext{
+resent addre:s: National Center for Physical Acoustics. L'niversity of Mississippi, Coliseum Drive. Lniversity, MS 386 it
}

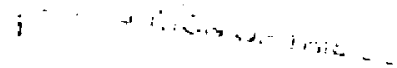




\section{Introduction}

In order to achieve improvement in the magnetic confinement properties of tokamak fusion reactors, it is useful to be able to calculate what types of equilibria are free from resistive MHD instabilities. In this paper we present an arcurate numerica] method in determine the resistive MHD stability of arbitrarily shaped axisymmetric lorojolal equilibria. The mathematical problem requires solving a sy-stem of two-dimensional partial differential equations in which the complex frequency of the resistive MHD eigenmodes is the eigenvalue.

Obtaining an accurate numerical solution to these equations is made difficult by several effects. In typical systems of interest the resistivity plays the role of a small parameter multiplying the highest derivative, leading to the presence of an internal boundary layer in the solution. Also; there exists several classes of solutions whose eigennodes are of widely different polarization, and whose eigenvalues can differ by many orders of magnitude. Resistive instabilities correspond to one of the smallest eigenvalues and the most singular eigenfunctions.

The time scale for ideal instabilities in tokamaks is given by the Alfien time, $\tau_{A}-$ $a \sqrt{\rho} / B$ (in rationalized MKS units). Here, $a$ is the plasma minor radius. $\rho$ is the plasma density, and $B$ is the magnetic field. The slow time scale of global resistive diffusion is given by $\tau_{H}=a^{2} / \eta$, where $\eta$ is the plasma resistivity. In the limit as $S=\tau_{H} / \tau_{A} \rightarrow x$. a boupdary laver theory has been developed in which one dimensional "inner region" equations involving resistivity are solved within the boundary laver, and twr-dimensimal "outer region" equations without resistivity are solved away from the boundary layer. These regions are connected by asymptotic matrhing of the solutions from the neighboring regions.

By using this matching technique, the asymptotic growth rate scaling for various resistive modes can be derived. FKR $[3$ discovered the growth rate scaling of the tearing mode. $\tau_{A}^{3 i 5} \tau_{A}^{2 / 5}$. and the sesistive interchange mode sraling. $\tau_{R}^{1 / 3} \tau_{A}^{2 / 3}$. The asymptritic matching method has bees implemented numerically in cylindrical geometry 9 , and has been used to calculate the resistive stability properties of cylindrical equilibrium with arbitrary profiles.

Cilasser, Greene, and Johnson is extended the analyical asymptotic analysis to arbitrarily shaped axisymmetric toroidal plasma equilibria. The asymptotic matching approach was successfully used to obtain a qualitative theoretical understanding of toroidal effects on the stability of a plasma against resistive modes. GGJ found that farorable average curvature is an important stabilizing 20 effect.

Although the asymprotic approach established a qualitative theory of toroidal effects. it has proven quite difficuli to apply this approach to deterinine the stability of arbit rary axisymmetric toroidal equilibria. The resistive PEST-3 cude was successful only in examining the stability of pressure-free circular tokamak equilibria with one 2 or more 6. 
rational surfaces. Additional asymplotic matching codes are presently being developed $T-9$.

Initjal value codes have been developed in order to solve the time dependent linear for non-linear) resistive $\mathrm{MHD}$ equations throughout the entire plasma $[4,10-14$. For toroidal equilibria. these codes are typically effective for $S \leq 10^{6}$. Higher values of $S$ are limited by numerical accuracy and/or arailable computing resources. The $S$ regime relevant tr. fusion reaciors is given by $10^{8} \leq S \leq 10^{10}$.

Eigenvalue or "spectral" codes have been developed $15-16$, 10 solve the full set of linparized equations throughout the entire plasma, but these codes are specific to crlindrical equilibria and are typically limited to $S \leq 10^{\overline{7}}$. Kerner was able to create a cylindrical eigenvalue code which succeeded in obtaining resilis for $S \leq 10^{10}$ by taking measures to reduce the fast wave "spectral pollution" problem [17]. However, there is got a strajghtforward way to extend Kerner's technique for eliminating pollution from the cylindrical problem to the toroidal problem.

It is a challenging task to find a satisfactory method of solving the toroidal stability problem in a numerically efficient manner. for reasons that we now explain. Physically. the rulc of resistivity is to allow the plasma to move with new degrees of freedom that are forbidden by the jdeal MHD equations. One of the linearized resistive MHD equations can be written as

$$
\left(1-\frac{\eta}{s} \Gamma^{2}\right) \dot{b}=\Gamma \times(\vec{\xi} \times \vec{B})
$$

where $\vec{\xi}$ is the plasma displacement, $s$ is the growth rate, and $\vec{B}, \vec{b}$ respectively give the equilibrium and perturbed magnetic fields. If the plasma perturbation has spatial varjations over lengths on the order of $L$, the maximal growth rate of a mode significantly affected by the resistive term in Eqn. (1.1) is on the order of $s \sim \eta / L^{2}$. In order for a growing mode to become jnteresting on a shorter time srale than the magnetic field diffusion time $\left(a^{2} / \eta\right)$, the mode must have spatial variations on a much smaller spatial scale than a. The satural candidates for jdeal MHD modes that will be significantli influenced by resistivity are then the continum modes, which diverge at singular surface(s), 3.18 .

The singular continuum modes arise when the highest order coefficient of the jdeal MHD radial displacement eigenmode equation vanishes. Calculating this leading order coefficient accurately is essential in order to resolve the continuum modes near the point where they diverge. The accurate numerical calculation of this small leading order term can be particularly difficult because of the presence of large fast wave terms. A small relative error in the computation of the large terms associated with the fast wave can "swamp" the computation of the singular behavior of the continuum modes. Therefore, one of the greatest challenges addressed by this paper is to find a way io formulate the resistive MHD equations which accurately resolves the continuum spectrum. We have chosen a mathematical formulation of the resistive MHD eigenmode equations that decouples the continuous spectra from the fast magnetosinic wave. This formulation is a generalization of the one previously employed by the NOVA $1^{\circ}$ code for ideal MHD.

The organization of this paper is now giverl. In Section II. we describe the numerical 
problem of spectral pollution. We present a scheme to avoid spectral pollution and to accurately resolve low frequency information by separating out the computation of the ideal MHD continuum spectrum away from the computation of the fast wave. Section IJI gives the equations developed for our formulation of the linearized MHD eigenmode equations. Section IV provides an understanding of the numerical methods employed by the NOVA-R code. Section V gives results from the NOVA-RC code which implements the ryndrical limil of our formulation of the resistive MHD stability problem. For a resistive interchange mode, we show that our results have four significant digit agreement with an asymptotic matching code at a magnetic Reynold's number of $S=10^{30}$. This represents a breakthrough for a non-asymptotic code, and justifies the approach that we have taken to eliminate numerical pollution from the fast wave. Results for resistive interchange and tcaring instabilities are given from the fully toroidal two dimensional NOVA- $R$ code in Section 11. The NOVA-R results are in excellent agreement with resistive PEST results for a zero $B$ case. A summary of the paper is given in Section V1. Appendix A and A ppendix $B$ discuss important technical issues relevant to insuring the acruracy of cubic B-spline packages when one wishes to closely pack grid points near an internal boundary layer.

\section{Spectral Pollution}

A resistive stability code must minimize the effect of fast wave spectral pollution if it is to be capable of investigating resistive modes for resistivities low enough to be in the range of intesest for fusion reactors. Here, we illustrate in the cylindrical limit why it is necessary for the problem to be formulated in terms of variables that "separate out" these continuum-Jike modes from the fast magnetosonic modes in order to more accurately resolve the continuum-like resistive modes of interest.

Consider a cylindrical plasma column in which the cylindrical coordinate $r$ is the only nun-ignorable coordinate. We define the differential operators $F=h_{:}=-i\left(\frac{\bar{B}}{\bar{P}}\right) \cdot \Gamma$ and $h_{s}=-i\left(\frac{\vec{B}}{B} \times \dot{r}\right) \cdot \Gamma$ which respectively correspond to directional derivatives along and across field lines but within the plane of the magnetic surfaces. The directional derivative across the surfaces is given by $k_{r}=-i \frac{\theta}{\partial_{r}}$. Let the simplifying assumption be made that $F=h$, varies with $r$ while all other equilibrium quantitjes are independent of $r$. Thus, only $F=F(r)$ will be in the way of recovering the homogeneous limit. In this quasi-homogeneous cylindrical limit, the linearized ideal $\mathrm{MHD}$ eigenmode equation for the radial displaccmert is $\left[19^{\circ}\right.$.

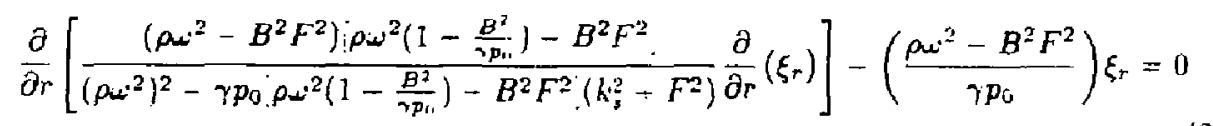

Here $\%=5 / 3$ is the ratio of specific heats. The coefficient of the highest order derivative 
will vanish if either

$$
\begin{gathered}
\rho \omega^{2}-B^{2} F^{2}=0 \\
\text { or } \rho \omega^{2}\left(1+\frac{B^{2}}{\gamma P_{0}}\right)-B^{2} F^{2}=0 .
\end{gathered}
$$

Equations (2.2) and (2.3) correspond to dispersion relatjons for the shear Alfvén and slow sound continuum, respectively. If there is any point in the plasma where Eqn. (2.2) or (2.3) is satisfied, then a singular eigenmode solution exists 20.

Among the regular solutions to Eqn. (2.1) is the fast magnetosonic wave. In the homogeneous limit for $k_{i 1}^{z}<<k_{-2}^{2}$, the fast wave dispersion relation is

$$
\rho \omega^{2}=\gamma p_{0}\left(1+\frac{B^{2}}{\gamma p_{0}}\right)\left(k_{\perp}^{2}+F^{2}\right)+O\left(F^{4}\right)
$$

where $k_{-}^{2}=k_{s}^{2}+k_{r}^{2}$. The eigenfrequency of the fast magnetosonic mode is much larger than that of all the other ideal MHD modes. For $\gamma p_{0}<<B^{2}$ the fast wave is given by' $\rho_{\mu^{2}} / B^{2} \approx k_{-}^{2}$ and $k_{-}^{2}=k_{s}^{2}+k_{r}^{2}$, so we subsequently refer to $k_{s}^{2}$ as a "fast wave term".

A formulation of the irieal MHD equations, which is vulnerable 1.0 spectral pollution. is now demonstrated. The plasma displacement, $\vec{\xi}$; and perturbed particle pressure. $p_{1}$. are used as unhnown variables. For $\xi_{s}=\left(\frac{\vec{B}}{B} \times \dot{r}\right) \cdot \vec{\xi} \cdot \xi_{r}=\dot{r} \cdot \vec{\xi}$ and $\left(x_{1}, x_{2}, x_{3}\right)=$ $\xi_{s}: B_{-} i p_{1} / B^{2}-\xi_{-}$", the "quasi-homogeneous" ideal $\mathrm{MHD}$ çlindrical limit equations are given by

$$
\left(\begin{array}{ccc}
-\frac{\rho \dot{H}^{2}}{B^{2}}-k_{s}^{2}-F^{2} & k_{s} & k_{s} k_{r} \\
k_{s} & \frac{B^{2} F^{2}}{\rho_{r^{2}}^{2}}-\frac{E^{2}}{\gamma p_{t}} & k_{T} \\
k_{s} k_{r} & k_{T} & -\frac{\rho w^{2}}{B^{2}}-k_{s}^{2}+F^{2}
\end{array}\right)\left(\begin{array}{l}
x_{1} \\
x_{2} \\
x_{3}
\end{array}\right)=\left(\begin{array}{l}
0 \\
0 \\
0
\end{array}\right)
$$

Afuer Fourier transforms are applied in the two ignorable coordinates $\theta$ and $z$. note that $\xi_{s}$ and $p_{1}$ can be algebraically eliminated in terms of $\xi_{r}$ : since the upper left hand comer $2 \times 2$ matrix has no $\frac{\partial}{\partial r}=i k_{r}$ differential operators and therefore can be inverted. Eliminating $\mu_{1}$ and $\xi_{s}$. one obtains an equation which the numerically generated $\xi_{T}$ solution will obev;

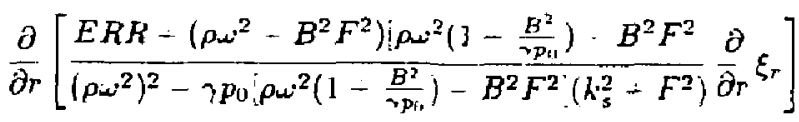

$$
\begin{aligned}
& -\left(\frac{\rho_{\alpha^{2}}-B^{2} F^{2}}{\gamma p_{0}}-\xi_{r}=0\right. \text {. }
\end{aligned}
$$

Equation (2.5) differs from (2.1) because of the ERR term. The ERR term represents the $k^{2}$ terms which cancel analytically but will not perfectly cancel computarionally. lumerical errors causing $E R R \neq 0$ will dirertly affect the accuracy of the continuum mode calculations. 
One contribution to the ERR term will come from the fact that the $k_{s}^{2}$ operalor in the uppes left corner element of the matrix in Eqn. (2.4) is not numerically equivalent to the product of two $k_{s}$ operators. This will resuit in a computation of the singularity associated with the shear Alfrén continuum given by

$$
\rho \omega^{2}=B^{2} F^{2}\left\{1+\frac{k_{s}^{2}}{F^{2}}\left[\frac{k_{s}^{2}-\left(k_{s}\right)\left(k_{s}\right)}{k_{s}^{2}}\right]\right\} \text {. }
$$

Here we define the numerical error due to the fast wave terms,

$$
E R R_{\perp}=\frac{k_{s}^{2}-\left(k_{s}\right)\left(k_{s}\right)}{k_{s}^{2}} .
$$

In toroidal geometry, the error due to $k_{s}^{2} \neq\left(k_{s}\right)\left(k_{s}\right)$ will be enhanced by the convolution errors caused by the truncation of the Fourier series representation of $k_{\mathrm{s}}$ and $k_{3}^{2}$. Keeping $M$ harmonics, the truncation error of Fourier harmonics gives $k_{s}^{2}-\left(k_{s}\right)\left(k_{s}\right) \sim e^{-d M / 2}$. where the constant $d$ is of order unity. When $k_{-}^{2} E R R_{-} \approx k_{1 !}^{2}$, the fast wave pollution becomes a serious problem.

We сал use an estimation for $E R R_{+}$based on the specific numerical computation being used. The numerical errors generated by $N$ linear elements in $r . N$ cubic elements in $r$. and $\mathrm{i}$ Fourier harmonics in $\theta$ are represented by

$$
E R R_{l}=a N^{-2}, E R R_{c}=b N^{-4}, E R R_{F}=c e^{-A d d / 2} \quad \text { respectively. }
$$

For our crude estimates, we take $a=b=c=d=1$. For tearing modes $u-\eta^{3 / 5}$ and at the resistive layer $\frac{k_{-1}}{k_{-}}-\frac{6}{q} \eta^{2 i 5}$. Here $t$ is the inverse aspect ratjo and we use $\frac{1}{q} \approx \frac{1}{10}$. The range of resistivities of interest to fusion reactors is $10^{-10}<\eta<10^{-8}$ in our units (where the resistivity is the inverse Magnetic Reynold's number). Insisting that the spectral pollution error be no larger than one percent of the correct value for this Shear continuum at the boundary of the resistive layer $\left(k_{-}^{2} E R R_{\perp} / k_{i}^{2}<.01\right)$, one obtains the numerical constraints given by: Table 1.

Table 1. Numeriral Convergence Estimates

$$
\begin{array}{ccccc} 
& & \frac{\eta=10^{-6}}{25,000} & \frac{\eta=10^{-8}}{160,000} & \frac{\eta=10^{-10}}{1,000.000} \\
\text { Linear Elements } & X> & 160 & 400 & 1.000 \\
\text { Cubic Elements } & N> & 160 & 40 & 55
\end{array}
$$

These rough estimates on the constraint for $N$ are somewhat pessimistic. Improvement is possible by using a non-uniform radial grid and packing grid points near the singular surface(s). But the required $M$ number for resolution in $\theta$ rannot be significantly lowered. The run time wili scale like $N \mathrm{M}^{3}$. so carrying 55 Fourier modes will be costly. Due to round off errors, the computer will obtain the given theoretical scaling of finite element 
errors only for sufficiently low $N$ and $M$. Thus, for a poorly chosen formulation it may be impossible to adequately lower the numerical error from the fast wave terms.

The NOTA-R formulation eliminates the possibility of any fast wave terms $\left(k^{2}\right)$ entering into the calculation of the continuum singularities by analytically separating out the terms relevant to the computation of the continuum singularities. In order to demonstrate this, the $\xi_{y}$, equation of the final formulation which we have chosen is now given.

In the ideal limit, the NOVA-R formulation is based on the dependent variables $P_{1}=$ $p_{1}-\vec{B} \cdot \vec{b}, \xi_{w}, \xi_{s}$ and $\Gamma \cdot \vec{\xi}$ where $\vec{\xi}=\xi_{w} \frac{\Gamma_{v}}{\psi^{\prime} !}+i \xi_{s} \frac{\bar{\theta} \times \Gamma \psi w}{B^{2}}+i \xi_{b} \vec{B}$ and $\dot{\psi}$ plays the role of the radial coordinate. The eigenmode equations i], are given symbolically by:

$$
\begin{aligned}
& \mathbf{E}\left(\begin{array}{c}
\xi_{s} \\
\Gamma \cdot \vec{\xi}
\end{array}\right)=\mathbf{F}\left(\begin{array}{l}
P_{1} \\
\xi_{\psi}
\end{array}\right) \\
& \frac{\partial}{\partial \omega}\left(\begin{array}{l}
P_{1} \\
\xi_{\psi}
\end{array}\right)=\mathbf{C}\left(\begin{array}{l}
P_{1} \\
\xi_{\psi}
\end{array}\right)+\mathbf{D}\left(\begin{array}{c}
\xi_{s} \\
\Gamma \cdot \vec{\xi}
\end{array}\right)
\end{aligned}
$$

Where $\mathbf{E}$. F. C. and $\mathbf{D}$ are $2 \times 2$ matrix involving only surface operators. it. no $\frac{\partial}{\partial_{2}}$ terms operating on the dependent variables. After eliminating the $\xi_{\text {s }}$ and $\Gamma \cdot \vec{\xi}$ variables by numerical!y inverting the $\mathbf{E}$ matrix, we have

$$
\begin{aligned}
& \operatorname{det}(\mathbf{E}) \frac{\partial}{\partial \psi} P_{1}=H_{11} P_{1}+H_{22} \xi_{v}, \\
& \operatorname{det}(\mathbf{E}) \frac{\partial}{\partial \psi} \xi_{\psi}=H_{21} P_{1}-H_{22} \xi_{\psi} .
\end{aligned}
$$$$
\text { where }\left(\begin{array}{ll}
H_{11} & H_{12} \\
H_{21} & H_{22}
\end{array}\right)=\operatorname{det}(\mathbf{E}) \mathbf{C}-\mathbf{D} \mathbf{E}^{c} \mathbf{F} \text { and } \mathbf{E}^{\mathrm{c}} \equiv \operatorname{det}(\mathbf{E}) \mathbf{E}^{-1}
$$

Sote that the $\frac{\partial}{\partial \psi}$ "terms have been "tagged" with the det(E) term. For spatial variatjons of $\xi_{x}$ to become infinite, $\operatorname{det}(E)$ must vanish and the continuum singularities will be zeros of $\operatorname{det}(\mathbf{E})$. The formulation has been carefully chosen in such a mannes that no fast wave terms $\left(k^{2}\right)$ are in the $\mathbf{E}$ matrix. Therefore, taking $\operatorname{det}(\mathbf{E})$ will immediately give the continuum mode singularities without relving on the cancellation of large fast wave terms. Thus. the formulation has separated out the computation of the continuum mods singularities from all of the fast wave terms.

The explicit form of the $\mathbf{E}$ matrix was fust derived by Cheng and Chance 1 and is given by

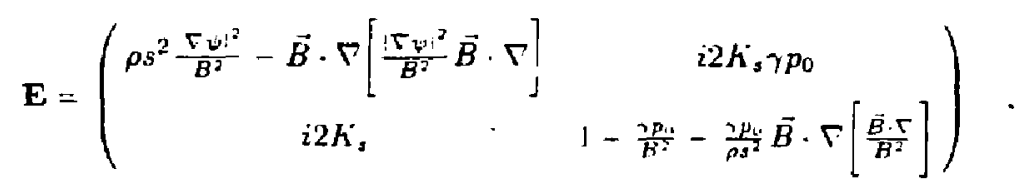




$$
\text { where } \vec{K} \equiv\left(\frac{\vec{B}}{B}\right) \cdot \nabla\left(\frac{\vec{B}}{B}\right) \quad K, \equiv \frac{\vec{B} \times \nabla \psi}{B^{2}} \cdot \vec{h} \text {. }
$$

The resistive MHD equations form a $6^{\text {th }}$ order system of differential equations in $\frac{\partial}{\partial \cdot}$. in Section II], we present the NUVA-R formulation. which reduces to the second order ideal MHD NOVA formulation in the limit as $\eta \rightarrow 0$. and thus eliminaies the fast wave suectral pollution of the lower frequency shear Alfien and slow sound continuous spectra.

\section{The NOVA-R Formulation}

\section{A. Equilibria}

Equilibria are constrained to sat iss!:

$$
\vec{J} \times \vec{B}=\Gamma P: \Gamma \times \vec{B}=\vec{j} \text { and } \Gamma \cdot \vec{B}=0 \text {. }
$$

Magnet ic coordinates have been used extensively in the literature to represent MHD equilibria 21-29: Our computation uses straight field line magnetic flux coordinates, given by $(2 \cdot \theta, \zeta)$. These coordinates are defined in terms of the cylindrical coordinates $(x, \dot{\phi}, Z)$. A stationary axisymmetric MHD equilibrium with isotropic pressure is describer in the form.

$$
p_{0}=P(\psi): \vec{B}=\Gamma \zeta \times \Gamma v+q(\psi) \Gamma \tau^{*} \times \Gamma \theta=\Gamma \phi \times \Gamma \dot{\psi}-g(v) \Gamma \phi
$$

where the magnetic flux is given by $\psi=\psi(\mathcal{X}, Z)$. The $\psi$ variable will be determined by the $\Gamma v$, component of the force balance equation. $\vec{J} \times \vec{B}=\Gamma F$, which gives a Poisson-like. partial differential equation called the equilibrium Girad-Shafranov equation.

$$
X^{-2} \Gamma \cdot\left[\frac{\nabla \psi}{X^{2}}\right]=-\left(x^{2} \frac{\partial P}{\partial \psi^{i}}+g \frac{\partial g}{\partial \psi}\right) .
$$

The Jacobian, $J \equiv\left(\nabla^{*} \times \nabla \theta \cdot \nabla \zeta\right)^{-1}$, is typically chosen such that for fixed $z$ and 0 a uniform $\theta$ grid corresponds to equidistant ares on the $v$ surface between adjacent grid points. For the most general case, we represent the Jacobian by

$$
J(X . Z)=\frac{x^{\prime}}{a(w)^{\prime} \Gamma w^{\prime j} B^{k}} \text {, where } i, j . \text { are integers. }
$$

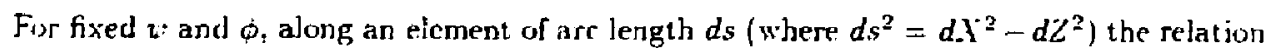

$$
\frac{d s}{d \theta}=\frac{J \Gamma z}{X}
$$


determines $\theta$, so specifying $J$ determines $\theta$. Here $\alpha(\dot{\psi})$ is determined by the constraint that $\theta$ be $2 \pi$ periodic. The straight field line toroidal angle coordinate is given by

$$
\zeta=\phi-q(\psi) \delta(\psi, \theta)
$$

where $\delta$ is $2 \pi$ periodic in $\theta$ and defined by the equation

$$
q(i)\left(1+\frac{\partial \delta}{\partial \theta}\right)=\frac{g J}{I^{2}} \text {. }
$$

This equation defining $\delta$ follows from the restriction that the field lines be straight in $(\vartheta, \theta, \zeta)$ coordinates, $i \epsilon, \vec{B} \cdot \Gamma \zeta / \vec{B} \cdot \nabla \theta=q(\psi)$.

\section{B. Resistive MHD Equations}

Here. a derivation of the equations solved by NOVA- $R$ is given. All perturbed quantilies have $e^{s t}$ time dependence where $s=-i$. The following vector equations have been indexed according to the equation number subsequently used to refer to their $\nabla_{w^{*}}, \vec{B} \times \Gamma_{\vartheta}$ aud $\vec{B}$ projections, respectivey: The linearized resistive MHD eigenmode equations (and two useful definitions) are given by:

$$
\begin{aligned}
& \rho s^{2} \vec{\xi}=-\Gamma p_{1}+(\Gamma \times \vec{B}) \times \vec{b}+\vec{j} \times \vec{B} \\
& \vec{b}=\Gamma \times(\vec{\xi} \times \vec{B}) \cdots(\eta / s) \vec{T} \\
& p_{1}+\nabla p_{0} \cdot \vec{\xi}+\gamma p_{0} \Gamma \cdot \vec{\xi}=0 \\
& \vec{T}=\Gamma \times \vec{j} \\
& \vec{j}=\Gamma \times \vec{b} \\
& \Gamma \cdot \vec{\xi} \equiv \Gamma \cdot\left\{\left(\frac{\vec{B} \vec{B}}{B^{2}}-\frac{(\vec{B} \times \Gamma v)(\bar{B} \times \Gamma v)}{B^{2} \Gamma \psi^{2}}+\frac{\Gamma v^{2}}{\Gamma \varphi^{2}}\right) \cdot \vec{\xi}\right\} \\
& \Gamma \cdot \vec{\xi}_{-} \equiv \Gamma \cdot\left\{\left(1-\frac{\vec{B} \vec{B}}{B^{2}}\right) \cdot \vec{\xi}\right\}
\end{aligned}
$$

While keeping all the $\Gamma \cdot \vec{\xi}$ terms, we now substitute in the following equations.

$$
\begin{aligned}
& p_{1}=P_{1}-\vec{B} \cdot \vec{b}
\end{aligned}
$$

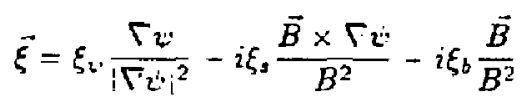

$$
\begin{aligned}
& \vec{b}=-i Q_{*} \cdot \frac{\Gamma_{z^{*}}}{\Gamma_{z^{*}}}+Q_{s} \frac{\vec{B} \times \Gamma_{z^{*}}}{\Gamma_{L^{2}}}-Q_{\times} \frac{\vec{B}}{\vec{B}^{2}}
\end{aligned}
$$




$$
\begin{gathered}
\vec{j}=-i R_{\psi} \nabla \psi+R_{\mathrm{J}} \frac{\vec{B} \times \nabla \psi}{|\nabla \psi|^{2}}+R_{b} \vec{B} \\
\vec{T}=-i T_{\psi} \nabla \psi+T_{2} \frac{\vec{B} \times \nabla \psi}{|\nabla \psi|^{2}}+T_{b} \vec{B}
\end{gathered}
$$

and use the following definitions:

$$
\begin{gathered}
' \equiv \frac{\partial}{\partial \psi}, \vec{K} \equiv\left(\frac{\vec{B}}{B}\right) \cdot \nabla\left(\frac{\vec{B}}{\vec{B}}\right) \\
K_{\psi} \equiv \nabla \psi \cdot \vec{K} \quad, \quad K_{s} \equiv \frac{\vec{B} \times \Gamma \psi}{B^{2}} \cdot \vec{K} \quad, \bar{S} \equiv\left(\frac{\vec{B} \times \nabla \psi}{|\nabla \psi|^{2}}\right) \cdot \nabla \times\left(\frac{\vec{B} \times \nabla \psi}{\mid \nabla \psi \psi^{2}}\right) .
\end{gathered}
$$

The 15 equations given by (3.1-3.10) and (3.1s-3.5s) are then modified by sequential]y substituting out $Q_{\psi}, Q_{b}, \nabla \cdot \vec{\xi}_{\perp}$, and $Q_{k}$ by using Eqn.s (3.1s) through (3.4s): respectively. Then, Eqn. (3.3) is modified by subtracting $1 / B^{2}$ times Eqn. (3.2). This removes the $\xi_{b}$ term in Eqn. (3.3). Finally, (3.5s) is used to eliminate $\xi_{b}$. The final resistive MHD equations to be implemented numerically in the NOVA-R code are given by the following ten equations.

$$
\begin{aligned}
& \rho s^{2} \frac{\Gamma v^{2}}{B^{2}} \xi_{s}-\dot{B} \cdot \nabla\left[\frac{\Gamma_{\psi^{2}}^{2}}{B^{2}}-\dot{B} \cdot \Gamma \xi_{s}\right]+i 2 K_{s} \gamma p_{01} \Gamma \cdot \ddot{\xi} \cdot i \dot{B} \times \Gamma v \cdot \Gamma\left[\frac{P_{1}}{B^{2}}\right] \\
& -\frac{\eta}{s} \frac{\vec{J} \cdot \vec{B}}{B^{2}} \mid \nabla \psi \mathrm{i}^{2} T_{1}-\frac{\eta}{s} i \vec{B} \cdot \Gamma T_{s} \\
& -i \frac{\vec{J} \cdot \vec{B}}{B^{2}} \vec{B} \cdot \nabla \xi_{\psi^{*}}-i \vec{B} \cdot \Gamma\left[\frac{\mid \nabla \psi^{2}}{B^{2}} \hat{S} \xi_{\tau^{\prime}}\right]+i 2 K^{\prime}, p_{0}^{\prime} \xi_{\downarrow^{\prime}}=0 \\
& i 2 K_{s} \xi_{s}+\left\{1+\frac{\gamma p_{0}}{B^{2}}-\frac{\gamma p_{0}}{\rho s^{2}} \vec{B} \cdot \nabla\left[\frac{\vec{B} \cdot \Gamma}{B^{2}}\right]\right\} \nabla \cdot \vec{\xi} \div \frac{P_{1}}{B^{2}} \\
& -\frac{\eta}{s} \frac{p_{0}^{\prime}}{\rho s^{2}} i \vec{B} \cdot \Gamma\left[\frac{|\nabla \psi|^{2}}{B^{2}} T_{t^{\prime}}\right] \div \frac{\eta_{1}}{s} T_{b}-\frac{2 K_{z^{\prime}}}{\mid \nabla \psi^{\prime 2}} \xi_{\mathrm{v}^{\prime}}=0 \\
& \frac{i}{B^{2}} \vec{B} \times \nabla i \cdot \nabla \xi_{s}+\frac{\gamma p_{0}}{B^{2}} \Gamma \cdot \vec{\xi}-\frac{P_{1}}{B^{2}}
\end{aligned}
$$

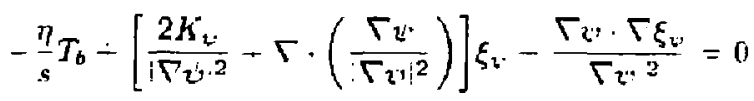

$$
\begin{aligned}
& T_{\psi} \div \frac{1}{\mid \bar{\nabla} \psi i^{2}}\left\{i \vec{B} \cdot \nabla R,-i \vec{B} \times \nabla i \cdot \nabla R_{b}\right\}=0
\end{aligned}
$$




$$
\begin{aligned}
& T_{s}+\frac{|\nabla \psi|^{2}}{B^{2}}\left\{\bar{B} \cdot \vee R_{w}-\dot{S} R_{3}-p_{0}^{\prime} R_{h}\right\}+\nabla \psi \cdot \nabla R_{s}=0 \\
& T_{b}+\frac{1}{B^{2}}\left\{-i \vec{B} \times \nabla \psi \cdot \nabla R_{\downarrow}-\left[p_{0}^{\prime}+\nabla \cdot\left(\frac{B^{2} \nabla \psi}{\mid \Gamma \psi t^{2}}\right)\right] R_{s}\right. \\
& \left.-(\vec{J} \cdot \vec{B}) R_{b}\right\}-\frac{\nabla \psi^{*} \cdot \Gamma R_{s}}{\nabla \psi \eta^{2}}=0 \\
& \vec{B} \cdot \nabla\left[\frac{|\nabla \psi|^{2}}{B^{2}} \vec{B} \cdot \nabla \xi_{s}\right] \div i \gamma p_{0} \vec{B} \times \nabla \psi \cdot \nabla\left[\frac{\nabla \cdot \vec{\xi}}{B^{2}}\right]+i \vec{B} \times \nabla \psi \cdot \nabla\left[\frac{P_{1}}{B^{2}}\right] \\
& -\underline{\eta}_{s} i \vec{B} \cdot \nabla T_{s}-\left.\nabla \psi\right|^{2} R_{\psi}+i \vec{B} \cdot \nabla\left[\frac{|\nabla \psi|^{2}}{B^{2}} \hat{S} \xi_{\psi}\right]+i p_{0}^{\prime} \bar{B} \times \Gamma \psi^{i} \cdot \nabla\left[\frac{\xi_{\psi}}{B^{2}}\right]=0 \\
& -\bar{S} \frac{\nabla \psi^{\prime 2}}{B^{4}} i \vec{B} \cdot \Gamma \xi_{\sigma}-\frac{p_{0}^{\prime}}{B^{2}} \frac{\gamma p_{0}}{B^{2}} \Gamma \cdot \vec{\xi}-\frac{p_{0}^{\prime}}{B^{2}} \frac{P_{1}}{B^{2}} \\
& -\frac{\eta}{s} \frac{i}{B^{2}} \vec{B} \cdot \nabla T_{b}-\frac{\eta}{s} \frac{\dot{S}}{B^{2}} T_{s} \\
& +\frac{R_{s}}{\mid \nabla \psi^{2}}-\left\{\left(\frac{p_{0}^{\prime}}{B^{2}}\right)^{2}-\hat{S}^{2} \frac{\mid \nabla \downarrow v^{2}}{B^{4}}+\frac{1}{B^{2}} \vec{B} \cdot \nabla\left[\frac{\vec{B} \cdot \nabla}{\mid \nabla \dot{\psi} \dot{\psi}^{\prime 2}}\right]\right\} \xi_{v} \\
& \rightarrow \frac{1}{\Gamma \psi \cdot 2} \Gamma \psi \cdot \Gamma\left\{\frac{\gamma p_{0}}{B^{2}} \Gamma \cdot \vec{\xi}-\frac{P_{1}}{B^{2}}-\frac{p_{0}^{\prime}}{B^{2}} \xi_{\psi}\right\}=0 \\
& -\left[p_{0}^{\prime}-\nabla \cdot\left(\frac{B^{2} \nabla \psi}{\nabla \psi \psi^{2}}\right)\right] \frac{\left.\nabla \psi\right|^{2}}{B^{-}} i \vec{B} \cdot \nabla \xi_{,}-\frac{\vec{J} \cdot \vec{B}}{B^{2}} \frac{\gamma p_{0}}{B^{2}} \Gamma \cdot \vec{\xi}-\frac{\vec{J} \cdot \vec{B}}{B^{2}} \frac{P_{1}}{B^{2}} \\
& +\frac{\eta}{s} \frac{i}{B^{2}} \vec{B} \times \Gamma \psi \cdot \Gamma T_{v},-\frac{\eta}{s} \frac{1}{B^{2}}\left[p_{0}^{\prime}+\Gamma \cdot\left(\frac{B^{2} \Gamma \psi}{\mid \Gamma \psi^{2}}\right)\right] T_{s} \\
& -R_{b}-\frac{1}{B^{2}}\left\{-p_{0}^{\prime} \frac{\vec{J} \cdot \vec{B}}{B^{2}} \div\left[p_{0}^{\prime}+\Gamma \cdot\left(\frac{B^{2} \nabla \psi}{\Gamma \psi_{i}^{2}}\right)\right] \frac{\Gamma_{\psi}^{\prime 2}}{B^{2}} \dot{S}-\vec{B} \times \nabla \cdot \cdot \Gamma\left[\frac{\vec{B} \cdot \Gamma}{\nabla \psi^{\prime 2}}\right]\right\} \xi_{\psi}
\end{aligned}
$$

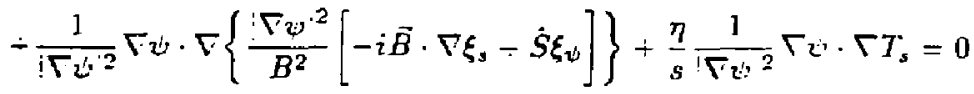

IU 


$$
\begin{aligned}
& \left(\frac{\vec{J} \cdot \vec{B}}{B^{2}}-\frac{|\nabla \psi|^{2}}{B^{2}} \dot{S}\right) i \vec{B} \cdot \nabla \xi_{s}-\gamma p_{0} \frac{2 K_{\psi}}{\mid \Gamma \psi_{\mid}^{2}} \Gamma \cdot \vec{\xi}-\frac{2 K_{\psi}}{|\nabla \psi|^{2}} P_{1} \\
& -\frac{\eta}{s} i \ddot{B} \cdot \nabla T_{\psi}-\frac{\eta}{s}\left(\frac{\vec{J} \cdot \vec{B}}{B^{2}}-\frac{\mid \Gamma \dot{\psi} !^{2}}{B^{2}} \hat{S}\right) \frac{B^{2}}{! \nabla^{\prime} \psi^{2}} \Gamma_{s}
\end{aligned}
$$

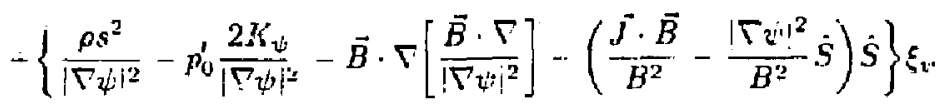

$$
\begin{aligned}
& -\frac{\nabla \psi \cdot \nabla P_{1}}{\left.! \nabla \psi\right|^{2}}=0
\end{aligned}
$$

These ten equations form tie NOVA-R formulation. The 10 dependent variables are

$$
\xi_{s}, \nabla \cdot \vec{\xi}, P_{1}, T_{\psi}, T_{s}, T_{b}, R_{\psi}, R_{s}, R_{b}, \xi_{\psi}
$$

In the limit where $\eta \rightarrow 0$, four of the ten NOVA-R equations, (3.1-3.3, 3.10), jdentically recover the NOVA formulation [1].

The resistive boundary conditions imposed at the wall are that $\xi_{t i}, R_{s}$ and $R_{b}$ vanish. It wiil be show that this is sufficiest to provide boundary conditions for all variables. The condition that $R_{s}$ and $R_{b}$ vanish follows from: 1) $\xi_{v^{n}}=0$ (impenetrable wall), 2) $\vec{v}_{0}=0$ (flow-free equilibria), 3) the tangential electric field vanishes at the (infinitel: conducting) wali, and 4) $\vec{E}+\vec{v} \times \vec{B}=(\eta / s) \vec{j}$. But if $\eta=0$, the fourth point in this argument breaks down =ince Ohn's law no longer yields any information about $\vec{j}$. In the jdeal limit. $\boldsymbol{R}_{s}=\boldsymbol{R}_{s}\left(\xi_{v}, \xi_{v^{\prime}}^{\prime}, \xi_{\dot{w}}^{\prime \prime}\right) \neq 0$ and $\boldsymbol{R}_{b}=\boldsymbol{R}_{\dot{v}}\left(\xi_{v}, \xi_{v}^{\prime}, \xi_{w}^{\prime \prime}\right) \neq 0$. For the ideol MHD problem one only impuses $\xi_{\psi}=0$ at the vall, and there is no additional freedom to prescribe $R$, arid $R_{\mathrm{b}}$ independently:

The additional, non-ideal boundary conditions at the wall have the potential of preventing the recovery of ideal $\mathrm{MHD}$ results in the $\eta \rightarrow 0$ limit. However. the NOlA-R formulation has the beneficial property of directly recovering ideal MHD results in the $\eta \rightarrow 0$ limit because the additional non-resistive boundary conditions fully decouple from the second order differential $\xi_{w}$ equation. This provides a smooth connection to ideal MHD in the appropriate limit.

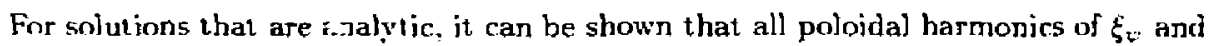
$R_{s}$ must ranish at the origin. All $m \neq 0$ poloidal harmonics of $R_{b}$ must also vanish, and for $m=0, \frac{\partial}{\partial x} R_{b}^{(0)}=0$ whese $x=\sqrt{\psi}$. This gives the set of bouadary conditions that are used in the NOVA-R code at the origin. 


\section{Numerical Method}

\section{A. Discretization}

Tis make further progress, the functional dependence of the problem on the poloidal angle $\theta$ and the toroidal angle $\zeta$ is discretized by expanding each of the 10 depencient variables into a truncated Fourier serjes. Only a single harmonic, $n$, in the toroidal angle js retained since the axisymmetric equilibrium does not mix modes of different $n$ number. However. the two-dimensional equilibrium does couple together polojdal harmonics associated with different $m$ values. We keep $M$ harmonics in the polojdal angle, letting $m$ vary from mlo to $m h i$, where $M=m h i-m l o+1$. After applying Galerkin's method to project out each of the $M$ Fourier harmonics, each analytical NOVA-R equation becomes $M$ coupled ordinary difierential equations. Each coefficient in the final analytical NOVA-R equations (3.1-3.10) becomes an $M \times M$ matrix which is in general $\psi$ dependent. Thesefore, all of the $M \times M$ coefficient matrices must be calculated on each $\psi$ surface (of the numerical 2 grid one has chosen).

Some of the coefficients in the $10 M$ by $10 M$ system of equations have terms which are multiplied by different powers of $s$, the growth rate. Each term involving a different factur of $s^{P}$ is computed independenty with $s^{P}$ factored out. Thus, the computation can be done oncs and for all outside of the loop in which one iterates over values of $s$ to find a solution.

Expanding into a Fourier series transforms the surface differential operators $\vec{B} \cdot \Gamma$ and $\vec{B} \times \Gamma v \cdot \Gamma$ into algebraic matrix multjpliers. This allows the first 7 of the 10 equations in the NOTA-R formulation to be algebraically inverted. thereby eliminating the first $\vec{\tau}$ unknowns (in the order as listed). To reduce the $10 \mathrm{M}$ equations in $10 \mathrm{M}$ unknowns into $3 . M$ equations in $3 M$ unknowns, first $\xi_{s}$ and $\Gamma \cdot \vec{\xi}$ are simultaneously eliminated by using Equn.s (3.1) and (3.2). Then, $P_{1}$ is eliminated using Eqn. (3.3). Nexi $T_{\psi}, T_{s}$ and $T_{b}$ are simultaneous]y eliminated using Eqn.s (3.4), (3.5) and (3.6), Finally, $R_{\psi}$ is eliminated using Eqn. (3.T). This procedure for reduction to a $3 M$ system of equations must be repeated on each $v$; surface. This reduction of the $10 M$ equations to $3 M$ is uniquely defined as long as it is understood that one never reduces terms by differentiating coefficients with respect to $z$. Thus. one would not make the replacement

$$
\frac{\partial}{\partial \psi}\left[E \xi_{\psi}: \rightarrow \frac{\partial E}{\partial \psi^{\prime}} \xi_{\psi}-E \frac{\partial \xi_{\psi}}{\partial \psi^{*}}\right.
$$

Making this type of substitution would typically create more work since new equilibrium quantities i.te. $\frac{\partial E}{\partial \psi}$, would have to be generated.

At this stage the $\operatorname{NOVA-R}$ formulation is a system of coupled second order radial differential equations for the $M$ Fourjer amplitudes of the three unknown quantities.

$$
\left(R_{s}, K_{b}, \xi_{w}\right)
$$


The numerical NOVA-R equations are of the form,

$$
\mathbf{C} \vec{y} \div\left[\overrightarrow{\mathbf{D}} \frac{\partial}{\partial \psi}\right] \vec{y}-\frac{\partial}{\partial \psi}[\mathbf{D} \vec{y}]-\frac{\partial}{\partial \psi}\left[\mathbf{E}\left(\frac{\partial}{\partial \psi} \vec{y}\right)\right]=0
$$

where

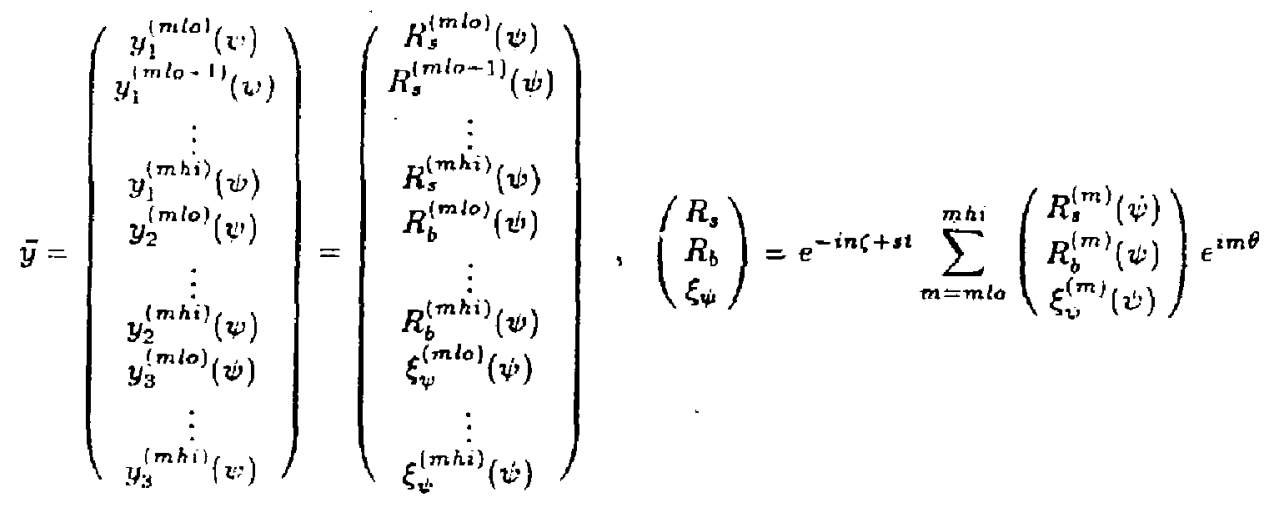

In the $\eta \rightarrow 0$ limit, the final $M$ equations represented in (4.1), originating from (3.10). identically recover the NOVA equations for $\xi_{v}$ and decouple from the remaining $2.1 /$ equations originating from (3.8) ard (3.9).

For,$Y=m h i-m l o+1$, the next step in the calculation involves discretizing this system of $3 M$ coupled second order differential equaiions in $\psi$ given by (4.1). An approximation for each poloidal harmonic of the unknown solution is to be constructed in the form.

$$
\left.y_{j}^{(m)} \quad i^{\prime}\right)=\sum_{k=1}^{N-2} y_{j}^{m k} B_{k}(v)
$$

One also approximates all of the coefficients, given on the left hand side of Eqn. (4.1). with a B.Splines expansion. This is done by evaluating all of the coefficients in Eqn. ( 1.1$)$ on "arts 2 . surface and then choosing an expansion for the coefficients that interpriates the $N$ data points. For our particular choice of finite element, the interpolation constraint senustes only. $r$ of the $N-2$ degrees of freedom. The remaining $t$ wo degrees of freedinn are found by using numerical approximations of the derivatives of each roefficient at the chrlpoints of the " grid.

In carrying out this procedure, one arrives at a tensor equation for the finite element anplit udes of the form, 


$$
\begin{gathered}
{\left[B_{i j}^{l m p k}(s)+C_{i j}^{l m p k}(s)+D_{i j}^{l m p k}(s)+E_{i j}^{l m p k}(s)\right] y_{j}^{m k}=0} \\
\text { for }\left(\begin{array}{c}
i, j \\
l, i m \\
p, k
\end{array}\right)=\left(\begin{array}{c}
1,2,3 \\
m l a, \ldots m h i \\
1, \ldots N-2
\end{array}\right) .
\end{gathered}
$$

Here $i$ refers to one of the three final NOVA-R equations originating from $(3.8-3.31)$. and $l$ and $p$ refer to $e^{-l \theta} B_{p}(\psi)$ projection of that equation. The $j, m, k$ indices are consistent with Eqn. (4.2).

At this point we have developed $3 M(N+2)$ equations to solve for $3 M(N+2)$ unknowns. For the cubic B-Splines, only $B_{1}(\psi)$ and $B_{N+2}(\psi)$ are non-trivial at $\psi=0$ and $i=1$ respeclively. Therefore, the boundary conditions, $y(t)=0, \theta)=0$ and $\vec{y}(\psi=1, \theta)=0$ are isatisfied by imposing the condition that $y_{j}^{m k}=0$ for $k=3$ and $k=N+2$ for all in and $j$. This represents a set of $6 M$ additional equations. These $6 M$ equations effectively remove the $B_{1}(\psi)$ and $B_{N+2}(\psi)$ finite elements from the basis set being used to expand the unknown independent variables. Galerkin's method requires that the solution be orthogonal to the basis functions used to expand the unknown functions. The $6 . M$ equations corresponding to the projections of $B_{1}(\psi)$ and $B_{N+2}(v)$ are, therefore. replaced by the boundary conditions.

By rearranging the indices, one can reformulate the lensor equatiu: given in Eqn. (4.3) into a simple matrix equation. The structure of the cubic B-spline finite element generated matrix is most easily visualized by considering each $3 M \times 3 M$ mat rix to be one "element", With this understond, when using $N$ grid points in $y$ : one generates an $(N+2) \times(N+2)$ matrix with seven non-trivial diagonals. The convention used is that for

$$
a=(N-1) M(p-1)+M(i-1)+l-m l o+1, \quad \beta=(N+2) M(k-1)+M(j-1)-m-m l o-1 .
$$

$$
\begin{gathered}
\mathbf{H}_{\mathrm{a}, \beta}(s) \equiv B_{i j}^{l m p k}(s)+C_{i j}^{l m p k}(s)+D_{i j}^{l m p k}(s)+E_{i j}^{l m p k}(s) . \\
\text { and } \zeta_{\beta} \equiv y_{j}^{m k} .
\end{gathered}
$$

Now the problem has been reduced to the single matrix equation.

$$
[\mathbf{H}(s)] \mathbf{H}^{\mathbf{*}}=\mathbf{0}
$$

In order 10 find non-trivial solutions, one iterates over complex s spare to find zeros of det $\mathbf{H}(s)$. Given a value $\bar{s}$ such that $\operatorname{det}[\mathbf{H}(\bar{s})]=0$, the associated eigenfunction solution corresponds to the null space of the $\mathbf{H}(\bar{s})$ matrix.

The eigenvalues, $\bar{s}$, that are real numbers are relatively easy to find. Since the determinant is real for all real $s$ : one simply needs to plot out values of the delerminant along the the real $s$ axis and look for sign changes. A binary search is then used to converge to the $\vec{s}$ value where the sign change occurs. For complex eigenvalues, solutions are much more difficult to find. Typically one is forced to start with an equilibrium in which the 
eigenvalue of interes! is real, and then slowly change parameters toward the desired equilibrium while tracking the root through the complex plane. As long is such a technique is used to insure that one knows an initial guess very near the complex eigenvalue of interest. then Muller's method may be used to converge to the precise value of $\bar{s}$.

\section{B. Numerical Advantages of the NOVA-R Formuiation}

A question this paper addresses is how one might best reduce the resistive eigenmode cquations into an equivalent workable sel of equations which a computer can accurately solve. We have chosen the NOYA-R formulation which is characterized by the following beneficial feaı ures:

1. NOVA-R involves the Fouries moments of three coupled second order equations in $N=3$ variables. The CPU time required to evaluate the determinant of the finite element generated matrix scales like $N^{3}$, so formulations solving for more unknowns wil] run slower. Furthermore, the determinant of a larger matrix will typically be a more iilbehaved function of $s$ so formulations using more than $N=3$ dependent variables will require more iterations of evaluating the determinant before converging to an eigenvalue.

2. Galerkin's method is optimally suited for stcond order equations and poorly suited for first order equations 30 . For finite resistivity and for $\eta=0$, none of the variables have highest order derivatives (in $\frac{a}{\partial \psi}$ ) which are first derivatives.

3. For $\eta=0$ NOVA- $\mathrm{R}$ directly recovers the identical formulation of a previously developed 2D ideal MHD code, NOVA. $A$ "smoolh connection" exists between the finjte $\eta$ $\mathrm{NOVA}-\mathrm{R}$ code and the NOVA code in the $\eta \rightarrow 0$ limit. because the NOVA-R formulation was deliberately formed in such a way that the non-ideal resistive boundary conditions become decoupled from the rejerant computation for the $\eta=0$ equations.

4. The NOVA-R equations do not become degenerate at any point in the plasma away from the magnetic axis.

\section{Cylindrical Geometry Results}

Substantial simplification of the formulation given in Sectior. 11 results when we specialize to "st raight" or $1 \mathrm{D}$ equilibria. Using cylindrical ( $r . \theta . z)$ crordinates. the equilibrium quantities depend only on the minor radjus $r$, and are thus one-dimensional. The only tornidal effect retained is the periodicity length, $0<z<2 \pi R_{0}$. When the $\theta$ and $z$ dependences are expanded in a Fourier series; $\epsilon . g$.

$$
\xi_{r}(r, \theta, z)=\sum_{m, n} \xi_{r}^{(m, n)}(r) \operatorname{expli}\left(m \theta-n=/ R_{0}\right) .
$$

we find that because the equilibrium quantties depend only on $r$. there is no coupling 
between the harmonics with different $m$ and $n$. This simplification was used in developing the 1D NOVA-RC code, which is significantly less cumbersome than the more complete $2 D$ NOWA-R code. We first report on results using NOVA-RC for two problems given in the literature.

Even if one chooses to only use one polojdal harmonic in the NOVA-R code. many fundamental difterences exist between the $1 D$ NOVA-RC code and the $2 \mathrm{D}$ NOVA-R rode. For the $1 \mathrm{D}$ problem. the computationa! lask of determining the equilibria jnvolves integrating simple first order differential equations in one variable. This task is performed exisemely accurately. For the $2 \mathrm{D}$ equilibria, a second order partial differential equation must be solved iteratively. All of the equilibrium data required to define the coefficients of the stability equations are directly calculated at each point on the stability grid for the ID problem. For the $2 \mathrm{D}$ problem, the required equilibrium data are interpolated from an equilibrium grid onto the stability grid. For the ID stability equations, every coeffcient is explicitly expressed as an analytical function of the equilibrium data. In $2 \mathrm{D}$, the coefficients of the final system of differential equations are generated numerically:

Wie do not give an explicit list of the initial NOVA-R formulation equations in the $1 \mathrm{D}$ limit. since it is preferable to instead refer to the more comprehensive $2 \mathrm{D}$ equations given by (3.1-3.10) and (3.1s-3.5s). In the $1 D$ limit, this list of 15 equations in 15 variables includes 6 firsi order differential equations and 9 zeroth order equations. These equations include 3i coeficients of undifferentiated independent variables and 6 coefficients of differentiated varjables. These 63 terms form the starting point of a symbolic algebra manipulator program. The "REDLCE" utility was used on the MFECCC Cray-XMP E-machine. The last 5 of these 15 equations, (3.1s) through (3.5s), are eliminated analytically in both the $1 \mathrm{D}$ and $2 \mathrm{D}$ formulations. For the $2 \mathrm{D}$ code, the first 7 equations are numerically eliminated in order to arrive at the final set of differential equations which form the NOVA-R formulation. For the 1D problem. however, the first 7 equations are also eliminated analytically.

Unless otherwise stated, the resistivities given in this paper are scaled in units of inverse magnetic Reynold's number and E. M. L. units are used. In order to scale the given growth rate, $s$, and resistivity, $\eta$, into other units for a fusion reactor, one would use $\bar{s}=\tau_{. A} s$ and $\dot{\eta}=\eta \tau_{A} / a^{2}$. where $t_{A}=a \sqrt{\rho} / B(0)$. Here $\bar{s}$ and $\bar{\eta}$ are dimensionless numbers which are fixed regardless of the particular values of $a, p, B(0)$.

\section{A. Resistive Interchange Instabilities in Cylindrical Spheromak-like equilibria}

The first equilibrium we consider for testing the NOVA-RC code is the cylindrical spheromak-like equilibrium examined in reference :4;. The equilibrium profiles are defined for $0 \leq r \leq 1$ by

$$
B_{\theta}(r)=\frac{k r}{g_{0}} \exp \left\{-\int_{0}^{r}\left[\frac{q q^{\prime}}{k^{2}}+2 r-\frac{\alpha\left(q^{\prime}\right)^{2} r}{8 k^{2}}\right]\left\{\left(\frac{q}{k}\right)^{2}-r^{2}\right]^{-1} d r\right\}
$$




$$
\begin{gathered}
B_{z}(r)=q(r) B_{\theta}(r) /(k r) \\
q(r)=q_{0}\left(1-r^{2}\right), \text { and } p(r)=\frac{a}{8} \int_{r}^{1} r B_{2}^{2}\left(\frac{q^{\prime}}{q}\right)^{2} d r .
\end{gathered}
$$

We examine an equilibrium with the parameters:

$$
40=1.6 . a=0.7, k=a / R=0.3, B_{z}(r=0)=1, \rho=1, a=1 \text {. }
$$

Note $4 \equiv B(0) /(a \sqrt{p})=1$ here. We compute the growth rate of a $m=n=2$ mode.

The equilibrium is stable to ideal MHD interchange instabilities for $0<a<1$, and is unstable to resistive interchange modes for all $\alpha>0$ [. The growth rate of the resistive interchange mode is determined to within four significant figures of the converged result with $N=200$ radial grid points when $\eta \geq 10^{-10}$. For the NOVA-RC results given in Table 2, we use $N=1001$ in order to investigate just how low we can reduce the resistivity before the NOVA-RC will stop converging toward asymptotic matching results.

Table 2.

$\eta \quad$ NOYA-RC growth rate Asymplotic Matching growth rate

$\begin{array}{ccc}10^{-4} & 2.2603 \cdot 10^{-2} & 3.3410 \cdot 10^{-2} \\ 10^{-5} & 1.3634 \cdot 10^{-2} & 1.5843 \cdot 10^{-2} \\ 10^{-6} & 6.8617 \cdot 10^{-3} & 7.2977 \cdot 10^{-3} \\ 10^{-7} & 3.2125 \cdot 10^{-3} & 3.2973 \cdot 10^{-3} \\ 10^{-8} & 1.4480 \cdot 10^{-3} & 1.4642 \cdot 10^{-3} \\ 10^{-9} & 6.3665 \cdot 10^{-4} & 6.3968 \cdot 10^{-4} \\ 10^{-10} & 2.7505 \cdot 10^{-4} & 2.7560 \cdot 10^{-4} \\ 10^{-11} & 1.1763 \cdot 10^{-4} & 1.1772 \cdot 10^{-4} \\ 10^{-12} & 5.0217 \cdot 10^{-5} & 5.0234 \cdot 10^{-5} \\ 10^{-13} & 2.1573 \cdot 10^{-5} & 2.1576 \cdot 10^{-5} \\ 10^{-14} & 9.3765 \cdot 10^{-6} & 9.3772 \cdot 10^{-6} \\ 10^{-15} & 4.1317 \cdot 10^{-6} & 41318 \cdot 10^{-6} \\ 10^{-17} & 8.3246 \cdot 10^{-7} & \\ \vdots & \vdots & 8.3247 \cdot 10^{-7} \\ 10^{-20} & 7.9818 \cdot 10^{-8} & \vdots \\ 10^{-25} & 1.6929 \cdot 10^{-11} & 7.9818 \cdot 10^{-8} \\ 10^{-30} & 3.6403 \cdot 10^{-12} & 1.6929 \cdot 10^{-30}\end{array}$

For large values of resistivity, $\eta \geq 10^{-4}$, we expect the asymptatic matching theory 10 ireak down, with leading order errors scaling as $\eta^{1 / 3}$. As $\eta$ decreases, the grow $t$ h rates from SOVA-RC and asymptotic matching agree to five significant figures. But when $\eta$ is further reduced. $\eta<10^{-25}$ : the agreenent becomes worse. However, even at a magnetic Reynold's 
number of $S=10^{30}$, NOVA-RC still reproduces the asymptotic matching growth rate to four significant digits. Attaining this degree of accuracy at low resistivities represents a breakthrough for a non-asymptotic code.

Figure 1 show's the eigenfunction behavior in arder of decreasing resist ivity, illustrat ing how the eigenfunctions from NOVA-RC and astmptotic matching converge together. The NOVA-RC independent variable, $\xi_{w}$, is divided by $B_{\theta}$ in order to enable comprarison with the asymptotic matching clependent variable, $\xi_{\mathrm{r}}$.

We now choose $\eta=10^{-10}$ for the cylindrical spheromak equilibrium to do convergence tests. We demonstrate the convergence of the NOVA-RC code with both a uniform and a nonuniform grid. The procedure for how the radial grid points are preferentially allocated near the singular surface is described in Appendix B. Table 3 specifies how the growth rate changes according to the number of grjd points that are used. Figure 2 demonstrates the $v^{-+}$convergence obtained when using a uniform grid. Note that for the nonuniform grid, the growth rate is within 0.2 percent of the converged result with only $N=100$ grid points.

Table 3. NOVA-RC Growth Rates at $\eta=10^{-10}$

\begin{tabular}{c} 
Tomuniform Grid \\
$\times$ grid points \\
\hline 50 \\
60 \\
80 \\
100 \\
150 \\
200 \\
300 \\
400 \\
500 \\
600 \\
700
\end{tabular}

Growth Rate

$2.6452 \cdot 10^{-4}$

$2.7619 \cdot 10^{-4}$

$2.7386 \cdot 10^{-4}$

$2.7443 \cdot 10^{-4}$

$2.7490 \cdot 10^{-4}$

$2.7499 \cdot 10^{-4}$

$2.7504 \cdot 10^{-4}$

$2.7504 \cdot 10^{-4}$

$2.7505 \cdot 10^{-4}$

$2.7505 \cdot 10^{-4}$

$2.7505 \cdot 10^{-4}$
Uniform Grid

I grid points Growth Rate

1400

1500

1600

1800

2000

2400

4000

6000

8000

10000
$2.3512 \cdot 10^{-4}$

$2.4393 \cdot 10^{-4}$

$2.5610 \cdot 10^{-4}$

$2.6259 \cdot 10^{-4}$

$2.6940-10^{-4}$

$2.7382 \cdot 10^{-4}$

$2.7504 \cdot 10^{-4}$

$2.7505 \cdot 10^{-4}$

$2.7505 \cdot 10^{-4}$

$2.7505 \cdot 10^{-i}$

\section{B. Tearing Modes in Tokamak-like Equilibria}

Izzo. et al 12 reported on a tokamak-like jdeal $\mathrm{MHD}$ stable cylindriral equilibrium which was examined for stability against resistive tearing modes. The NOV: 4 - RC stability results are to be compared with the published results from the initial value rode used by Jzzo, referred to as HILO. HILO filters out the fast magnetosonir wave by naking an expansion in the inverse aspert ratio and the plasma $\beta$. The value of $\beta$ is assumed to be first order in $c=a / R$. The MHD equations HILO solves are accurate to fift horder in $t$. lzzo defines $\beta=2 p(r) / B^{2}(1)$ and for this case $c=0.2$ and $\beta \leq 6.13 \cdot: 0^{-4}$. 
The equilibrium of interes: is defined for $0 \leq r \leq 1$ by

$$
\begin{gathered}
g(r)=1.6\left(1+r^{2} / 0.64\right), \\
p(r)=\alpha\left(0.001+0.028 r^{2}-0.059 r^{4}+0.03 r^{6}\right) . \\
a=0.25, k=a / R=0.2, B_{i}(1)=1,0=1, a=1 .
\end{gathered}
$$

For the perturbation, $m=2$ and $n=1$ are chosen. This type of pressurc profile is unusual in that it has a positive gradient for $0<r<0.558$. In particular, $p^{\prime}\left(r_{s}=0.4\right)=$ $2.28 \cdot 10^{-3}>0$ at the resonant surface. The physical motivation for using an equilibrium with the pressure gradient reversed at the resonant layer was to simulate the effects of good average curvature.

Mie temporarily defer investigating this specific equilibrium by first choosing to investigate the simplet equilibrium generated by setting $\alpha=0$, thereby "turning of " the pressure profile. For this pressure-free equilibrium, we can check whether or not the growth tate computed by the NOVA-RC code will scale like $\eta^{3 / 5}$, which is predicted by asymptot ic theory. A tolal of $N=1001$ grid poirts is used to obliviate the need for numerical convergence studies.

\section{Table 4.}

NOVA-RC growth rate

\begin{tabular}{ccc}
$\underline{\eta}$ & $\underline{s}(\eta)$ & $\log _{10}\left[\frac{s(10 \eta)}{s(n)}\right]$ \\
\cline { 3 - 3 } $10^{-4}$ & $4.90904 \cdot 10^{-4}$ & \\
$10^{-5}$ & $9.53777 \cdot 10^{-4}$ & \\
$10^{-6}$ & $4.60722 \cdot 10^{-4}$ & .31601 \\
$10^{-7}$ & $1.49220 \cdot 10^{-4}$ & .48961 \\
$10^{-8}$ & $4.18112 \cdot 10^{-5}$ & .55253 \\
$10^{-9}$ & $1.10219 \cdot 10^{-5}$ & .57904 \\
$10^{-10}$ & $2.82845 \cdot 10^{-6}$ & $.5907]$ \\
$10^{-11}$ & $7.17215 \cdot 10^{-7}$ & .59590 \\
$10^{-12}$ & $1.80904 \cdot 10^{-7}$ & .59820 \\
$10^{-13}$ & $4.55230 \cdot 10^{-8}$ & .59922 \\
$10^{-14}$ & $1.14438 \cdot 10^{-8}$ & .59966 \\
$10^{-15}$ & $2.87554 \cdot 10^{-9}$ & .59985 \\
$10^{-16}$ & $7.22714 \cdot 10^{-10}$ & .59975 \\
$10^{-17}$ & $1.81469 \cdot 10^{-10}$ & .60016 \\
$10^{-18}$ & $4.58671 \cdot 10^{-11}$ & .59730
\end{tabular}

The results shown in Table 4 show the growth rate, $s(\eta)$, and its scaling with respect to resistivity for a wide sange of $n$ values. The convergence to the $3 / 5 \mathrm{scaling}$ begins to break down at about $\eta=10^{-16}$. This breakdown occurs when the growth rates reach about the same order of magnitude as the point at which NOVA-RC convergence to asymptotic 
matching began to break down in the previous jnterchange case. Figure 3 shows the tearing mode eigenmode for various values of the resistivity:

Again, the actual equilibrium from the lzzo paper is deferred in order to investigate what happens to the resistive tearing mode at a fixed resistivity as the pressure profile is gradually "turned on". The pressure profile is gradually increased by rarying the n parameter. We are thus able to confirm that the NOVA-RC code agres with Izzos:s demonstration that this tyje of pressure profile has a stabilizing effect on the mode. The particular value of the resistivity chosen is just above the point where the growth rate of the mode found bi NOVA-RC goes complex for an $\alpha=0.5$ pressure profile. For a fixed equilibrium, the "critical resistivity", $\eta_{c}$, refers to the value of $\eta$ below which the growth rate makes a transition from being purely real to complex. Fnr a fixed value of $\alpha=0.5$, the NOVA-RC code finds that the growth rate goes complex when the resistivity is less than $\eta_{c}=3.34 \cdot 10^{-7}$. (Note that $a=0.5$ is double the $\alpha=0.25$ case which lzzo investigated).

Table 5 .

$$
\eta=3.5 \cdot 10^{-7}
$$

$\begin{array}{lc}\underline{\alpha} & \text { NOVA-RC. growth rate } \\ .00 & 2.832 \cdot 10^{-4} \\ .10 & 2.389 \cdot 10^{-4} \\ .20 & 1.928 \cdot 10^{-4} \\ .30 & 1.450 \cdot 10^{-4} \\ .40 & 9.608 \cdot 10^{-5} \\ .45 & 7.027 \cdot 10^{-5} \\ .48 & 5.247 \cdot 10^{-5} \\ .50 & 3.535 \cdot 10^{-5}\end{array}$

Table 5 shows that the growth rate decreases as a jincreases and the effect of the pressure profile is stabilizing. Figure $A$ shows the unstable eigenmode for various values of $\alpha$.

Vote that the radial displacement develops a relative minimum in the laver as the $(\alpha, \eta)$ parameters approach the values for which the growth rate becomes complex. As the two mos: unstable growth rates coalesce. their corresponding eigenfunctions also coalesce. and the less unstable eigenfunction typically has this extra oscillation. (Here $\eta=3.5 \cdot 10^{\top}$ at $\alpha=0.5$ is near $\eta_{c}=3.34 \cdot 10^{-7}$ ).

We now fix $a=0.25$ so that we can compare the $10 \mathrm{VA}-\mathrm{RC}$ growth rates wit $\mathrm{t}$ hose given by Izzo. For resistivities in the range $1.6 \cdot 10^{-8}<\eta<2 \cdot 10^{-6}$ comparison with the HILO results from Izzo is given in Table 6. 
Table 6.

\section{NOVA-RC growth rate HILO growth rate}

$\begin{array}{lll}2 \cdot 10^{-6} & 3.41 \cdot 10^{-4} & 3.4 \cdot 10^{-4} \\ 6 \cdot 10^{-7} & 2.21 \cdot 10^{-4} & 2.2 \cdot 10^{-4} \\ 1 \cdot 10^{-7} & 7.94 \cdot 10^{-5} & 8.2 \cdot 10^{-5} \\ 3 \cdot 10^{-8} & 3.03 \cdot 10^{-5} & 3.2 \cdot 10^{-5}\end{array}$

Unfortunately, a comparison of the third decimal place with the lzze results is not possible because the HILO code is not able to find converged results to this degree of accuracy. The NOVA-RC code does agree with HILO at high resistivity to within the accuracy of the HILO corle it self.

For lower resistivities the complex part of the HILO growth rate is not given. As the the resistivity is lowered below $1.6 \cdot 10^{-8}$ we find that: 1) lowering the resistivity will eventually induce the osculation of the largest growth rate with the growth rate of a less unstable mode. 2) further reduction of the resistivity (after the osculation) will cause the unstable modes to "split" into two modes having respertive complex growth rates that are complex conjugates, and 3 ) ihese two modes subsequently become stable as the resistivity is further reduced.

In Table 7 we explicitly write out the results with complex growth rates for small values of $\eta$. A total of $N=300$ grid points was used for this study.

Table 7. Results at $a=0.25$

NOVA-RC growth rate

$\begin{array}{ccc}\underline{\eta} & \underline{\text { Real}(\mathrm{s})} & \underline{i * \operatorname{lmag}(\mathrm{s})} \\ 1.504 \cdot 10^{-8} & 8.54 \cdot 10^{-6} & =i 0.22 \cdot 10^{-6} \\ 1.50 \cdot 10^{-8} & 8.52 \cdot 10^{-6} & =i 0.68 \cdot 10^{-6} \\ 1.45 \cdot 10^{-8} & 8.22 \cdot 10^{-6} & =i 2.36 \cdot 10^{-6} \\ 1.4 \cdot 10^{-8} & 7.92 \cdot 10^{-6} & =i 3.24 \cdot 10^{-6} \\ 1.3 \cdot 10^{-8} & 7.31 \cdot 10^{-6} & \pm i 4.47 \cdot 10^{-6} \\ 1.2 \cdot 10^{-8} & 6.69 \cdot 10^{-6} & \pm i 5.37 \cdot 10^{-6} \\ 1.1 \cdot 10^{-8} & 6.04 \cdot 10^{-6} & =i 6.09 \cdot 10^{-6} \\ 1.0 \cdot 10^{-8} & 5.39 \cdot 10^{-6} & =i 6.67 \cdot 10^{-6} \\ 0.9 \cdot 10^{-8} & 4.71 \cdot 10^{-6} & =i 7.15 \cdot 10^{-6} \\ 0.8 \cdot 10^{-8} & 4.01 \cdot 10^{-6} & =i 7.53 \cdot 10^{-6} \\ 0.7 \cdot 10^{-8} & 3.29 \cdot 10^{-6} & =i 7.83 \cdot 10^{-6} \\ 0.6 \cdot 10^{-8} & 2.55 \cdot 10^{-6} & \pm i 8.03 \cdot 10^{-6} \\ 0.5 \cdot 10^{-8} & 1.78 \cdot 10^{-6} & =i 8.13 \cdot 10^{-6} \\ 0.45 \cdot 10^{-8} & 0.05 \cdot 10^{6} & =i 8.14 \cdot 10^{-6}\end{array}$


The HILO code found that the equilibrium became stable at (roughly) $\eta=4 \cdot 10^{-9}$. The NOVA-RC code, however, found stability at $\eta=4.5 \cdot 10^{-9}$. In arldition to HlLO. lzzo used a shooting code that solved the incompressible equations. By using the shooting code. Jzzo determined that the point at which the growth rate first became complex (as $\eta$ is lowered) nccurred at $(\eta, s)=\left(1.6 \cdot 10^{-8}, 9.6 \cdot 10^{-6}\right)$. The NOYA-RC code found $(7 . s)=\left(1.5 \cdot 10^{-8}, 8.6 \cdot 10^{-6}\right)$. Thus. small but noticeable differences exist between the results of lzzo and the more exact treatment in NOVA-RC at low resistivitits.

\section{Toroidal Geometry Results}

\section{A. Ideal Limit}

In this section we test the NOVA- $R$ code against several previously known results in turcidal geometry. The first test is to insure that the NOVA-R code correctly recovers known resulis in the axisymmetric $2 \mathrm{D}$ ideal MHD limit. As mentioned in the introduction. the NOVA-R formulation was deliberately chosen in order to greatly simplify this task. The NOV:A-R formulation has been chosen to insure that when one sets $\eta=0$, the NOVA-R code should recover results identical to that of NOVA.

Here we illustrate the numerical recovery of a published NOVA result with the NOVA$R$ code. An equilibrium, which the ideal NOVA code found to be unstable to an $m=1$, $\eta=1$ internal kink mode $1 j_{j}$; 5 analyzed by the NOVA-R code with $\eta=0$.

Tlic equilibrium has a circular outermost surface and is defined on the interval $0<$ $.4<$ I by profiles of the form

$$
\begin{gathered}
P(y)=P_{0}\left(1-y^{2}\right)^{2} \\
q(y)=g_{0}+y\left[q_{1}-g_{0}-(y-1)\left(g^{\prime}-g_{1}+q_{0}\right) \frac{1-y_{s}}{y-y_{s}}\right],
\end{gathered}
$$

where $y=2 / \Delta v$ and $y_{s} \equiv\left(q_{1}^{\prime}-q_{1}+q_{0} / q_{0}^{\prime}-q_{1}^{\prime}-2\left(q_{1}-q_{0}\right)\right.$. The equilibrium has $u_{u}=v^{\prime}{ }_{i m}-v_{0}=0.0609,<j>_{a r}=0.01277, R=1.43 . R / a=3.4, P_{0}=0.02456$. $g_{n}=0.8, q_{1}=2.85, q_{0}^{\prime}=13.857, q_{1}^{\prime}=106.88$, and $\left\langle\beta_{\text {pol }}\right\rangle=0.86$. We normalize the growth ratc in $w_{A} \equiv B_{0} /\left[q_{1} R p^{: .5}\right.$. for a vacium toroidal field given by $B_{0}=B_{0} R_{0} / R$. In specific. $B_{0}=\mathrm{J}$ and $\omega_{A}=0.2455$ here. Figure 5 gives the $\mathbf{0} 0 \mathrm{~A}-\mathrm{R}$ result obtained with $I=100$ evenly spaced \& grid points and ejght poloidal harmonics from mis $=-210$ $m h i=5$. For $\eta=0$, we have numerically verified the equivalence of all terms in XOYA-K with the corresponding NOVA terms. Figure 5 shows the eigenfunction $\xi_{v}$, for different polojdal harmonics corresponding to the growth rate, $\bar{s}=s / \omega_{-1}=4.8 \cdot 10^{-2}$. 


\section{B. Resistive Interchange Modes In Toroidal Spheromak Equilibria}

Our aim is to define a sequence of $2 \mathrm{D}$ toroidal axisymmetric equilibrium which allows 1he NOIA-R code 10 recover results from the $1 \mathrm{D}$ NOVA-RC cylindrical cride as the limit "sf the sequence. Let $S_{r}$ symbolically represent the system of three second order differential equations which make up the cylindrical stability equations. The cylindrical stability equations depend on the $q$ profile, the machine size, and the corvidal and poloidal mode numbers, $n, m$, of the perturbation. Clearly, $S_{c}=S_{c}(g, a, R, n, m)$. For $k=a / R$ the dependence of $S_{r}$ on $q, a, R, n, m$ can be written in the more restrictive form, $S_{c}=S_{c}(q / k, n k, m)$ 14. This means that one cannot recover the cylindrical linji with an axisymmetric $2 \mathrm{D}$ code by merely letting $k=a / R \rightarrow 0$. One also has to preserve the quantities $\eta / k$ and $n k$.

The following parameters specify the cylindrical spheromak-ike equilibrium which is being targeted for convergence studies. The equilibrium has a circular outermost surface and is defined in the region from $0 \leq r \leq 1$ with the parameters:

$$
\begin{gathered}
q(r)=q_{0}\left(1-.9 r^{2}\right), \quad p(r)=\frac{\alpha}{8} \int_{T}^{1} r B_{i}^{2}\left(\frac{q^{\prime}}{q}\right)^{2} d r ; \\
g_{0}=0.1, a=0.1, k=a / R=0.1, \quad B_{2}(0)=1, \rho=1, a=1 .
\end{gathered}
$$

The equilibrium has a centrally peaked pressure profile with $p(0)=5.71 \cdot 10^{-3}$. For the perturbation, $m=1$ and $n=15$ are chosen. Now we define a sequence of $2 D$ equilibria for which the corresponding 2D stability equations should approximate the $2 D$ stability: equations of the given cylindrical equilibrium as $a / R \rightarrow 0$. For low $G, q$, and $a / R$ in axisymmetric equilibria. the flux surfaces of the equilibria are very circular and the volume within a given flux surface, $V=V(\psi)$, should vary as $r^{2}$, where $r$ represents the average minor radius on the flux surface. After normalizing both $0 \leq r \leq 1$ and $0 \leq 1(2) \leq 1$. we define

$$
<r^{2}>\equiv \frac{\int_{0}^{2 \pi} r^{2} J / A d \theta}{\int_{0}^{2 \pi} J / A d \theta} .
$$

The 2D spheromak equilibria are specified by the following parameters and profiles:

$$
\begin{gathered}
q(2)=q_{0}(1-.91) . \quad \frac{d P}{d v}=-\alpha 1 \cdot \frac{d}{4} \frac{d V}{d \psi} B_{z}^{2}\left(\frac{1}{q} \frac{d \varphi}{d l}\right)^{2} . \\
0=0.1 . \quad B_{z}(0)=1 . \quad \rho=1, \quad a=1 .
\end{gathered}
$$

The pressure is required to vanish at the wall. Given the assumption that $l^{\circ}=r^{2}$. the cylindrical pressure profile is easily recovered after multiplying both sides of the equation for $\frac{d t}{d z}$ by $\frac{d t}{d r}$. The three sets of parameters shown in Table 8 were used to ohiain the large aspect ratio equilibria that we investigated. 
Table 8.

$\begin{array}{ccccc} & \underline{k=a / R} & \underline{q_{0}} & \underline{n} & \text { Resulting } p(0) \\ \text { Case 1 } & 0.25 & 0.25 & 6 & 5.54 \cdot 10^{-3} \\ \text { Case 2 } & 0.10 & 0.10 & 15 & 5.69 \cdot 10^{-3} \\ \text { Case 3 } & 0.01 & 0.01 & 150 & 5.73 \cdot 10^{-3}\end{array}$

For the third case, note that $p(0)=5.73 \cdot 10^{-3}$ is approximately the same as the value $\left(5.71 \cdot 10^{-3}\right)$ found in the eylindrical limit. For the purposes of testing whether NOVA$\mathrm{R}$ correctly recovers the cylindrical limit, the third case is of the most interest since the central pressure agrees well with the cylindrical limit and the inverse aspect ratio is small. Figure 6 gives the structure of the fiux surfaces on the radial $(\psi)$ stability computational grid for the $a / \boldsymbol{R}=0.01$ equilibrium.

First the NOVA-R code is tested when keeping only the resonant harmonic, ( $m=$ $1 . n=150$ ). This represents the closest possible comparison of NOVA-R with the computation periormed by the cylindrical code. Let $\left(N_{s}^{*}, M\right)$ represent the number of $\xi:$ ratial grid points and poloidal harmonics kept in the final NOVA- $R$ stability calculation. Let $t$ designate the width of a region or "layer" about the resonant surface. Lei $f$ represent the fraction of the total number of radial stability grid points $\left(N_{s}\right)$ which are to be packed within the layer. Let $\left(N_{e}^{\psi}, N_{e}^{\theta}\right)$ give the number of radial and theta grid points used in the equilibrium calculation and $\delta$ represent the error tolerance of the equilibrium code 27 . For the moment we are keeping only one poloidal harmonic, $(M=1)$, but in general, the growth rate is a function of many computational parameters.

$$
s=s\left(N_{c}^{\dot{v}}, N_{e}^{\theta}, \delta, N_{t}^{\psi}, M_{i}, f, \mathrm{c}\right)
$$

In order to confirm the results, convergens: with respect to all of these parameters had to be verified. Excellent numerical convergence of $s$ was easily obtained with respect to all

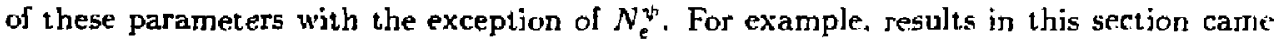
from $N_{s}=300$. and lowering $N_{s}$ to 200 changed only the sixth significant figure in the grow'th rate. Lowering $\delta$ by four orders of magnitude also affected only the sixth decimal of the growth rate. The surprisingly large dependence of $s$ on $N_{*}^{i}$ is shown in Fig. $\overline{7}$, which illustrates a $\left(N_{c}^{t *}\right)^{-2}$ dependence. The resistivity is fixed at $\eta=10^{-5}$ here. The data point given by a circlo indicates the cylindrical limit obtained from the NOVA-RC code. The growth rates given here are in units of $s=s / \omega_{A}$ and $\omega_{A}=10.0$ for all three spheromak equiljibria given in Table 8 . Figure 8 shows the unstable eigenfunction. $\xi_{v}$, for $\eta=10^{-7}$ and $N_{r}^{* *}=200$.

The equiljbrium information is calculated with the use of "centered" finite difierence formulas which are accurate to second order. Thus the equilibrium data are known to converge like $\left(N_{t}^{\psi}\right)^{-2}$. The tearing mode examined in Section C displays a relatively: weaker dependence on $N_{c}^{*}$. A decrease of 25 percent in the growth rate between $N_{\psi}^{*}=6 \mathrm{~A}$ and $N_{t}^{*}=200$ was seen for this spheromak equilibrium at $\eta=10^{-\top}$. This decrease is only 3 percent for the tokamak equilibrium (analyzed in Section $\mathrm{C}$ ) at $\eta=10^{-8}$. The unusually 
large $\mathcal{N}_{e}^{\psi}$ dependence seen here can primarily explained by the fact that for these low growth rates, the plasma is very close to marginal stability. Therofore, the accuracy of the equilibrium data is being tested more stringenty by the NOVA- $R$ rode than is usually the case for stability codes which examine ideal instabilities, for example.

The next simple test of the NOVA-R code involves expanding the stability calculation in order to ieep track of more than one harmonic. For this nearly cylindrical equilibrium. the addition of extra non-resonant hamonics sheuld not affect the result. For the previous runs only the $m=1$ resonant harmonic was kept. The range of harmonics is expanded to in $\in-1,0.1 .2,3$. The resulting growth rates are given in Table 9 .

Table 9. $\quad \eta=10^{-7}$ NOVA- $\mathrm{R}$ growth rates

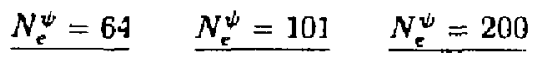

$\begin{array}{cccc}\text { Many Modes } & 4.236 \cdot 10^{-5} & 3.567 \cdot 10^{-5} & 3.146 \cdot 10^{-5} \\ \text { Single Modes } & 4.237 \cdot 10^{-5} & 3.568 \cdot 10^{-5} & 3.147 \cdot 10^{-5}\end{array}$

For an additional check we fix $N_{e}^{*}=200$ and fx the range of poioidal harmonics at $-1,0,1.2,3$ in order to examine the variation in the IOVA-R growth rate for each of the three previously defined equilibria cases, given in Tible 8. Toroidal effects are known to be of lesser importance for low $\beta, a / R$ and $q$ plasmas. This test, as shown in Table 10 , also helps to insure that the $a / R=0.01$ case was sufficiently converged to the $a / R \rightarrow 0$ limit so that we could properly compare it to the results from the cylindrical code.

Table 10. $\quad \eta=10^{-7}$ NOVA-R Growth Rates

$$
\underline{k=0 / R} \text { Growth Rate }
$$

$\begin{array}{lll}\text { Case 1 } & 0.25 & 3.34 \cdot 10^{-5} \\ \text { Case 2 } & 0.10 & 3.17 \cdot 10^{-5} \\ \text { Case 3 } & 0.01 & 3.15 \cdot 10^{-5}\end{array}$

Fur the final check we vary the resistivity while fixing $a / R=0.01, N_{e}^{\prime}=200$ and keeping the range of poloidal harmonics at $[-1,3]$. This is done to check whether $s \sim \eta^{1 / 3}$. The growth rates as a function of $\eta$ for the $a / R=0.01$ case are given in Table 11 and Fig. 9.

Table 11. $k=a / R=0$. (1) NOVA-R Growth Rates

$\begin{array}{cccc} & \underline{\eta=10^{-8}} & \underline{\eta=10^{-i}} & \underline{\eta=10^{-6}} \\ \text { NOVA-RC (Cylindrical Limit) } & 1.55 \cdot 10^{-5} & 3.22 \cdot 10^{-5} & 6.06 \cdot 10^{5} \\ \text { NOVA-R } & 1.58 \cdot 10^{-5} & 3.15 \cdot 10^{-5} & 5.90 \cdot 10^{-5}\end{array}$




\section{Tearing Modes In Tokamak Equilibria}

The following parameters give a tokamak equilibrium which has previously been determined to be unstable to resistive tearing modes by the resistive PEST code. This equilibrium has a circular outermost flux surface and is defined on the region from $0 \leq 2^{i} \leq 1$ with the parameters:

$$
\begin{gathered}
k=a / R=0.1, \quad B_{\phi}(0)=1, \quad \rho=1, \quad a=0.1, \\
q(\psi)=1.1+1.8 \psi^{2}, \quad p(\psi)=0 .
\end{gathered}
$$

For the computation of the equilibrium, $N_{e}^{\mu}=200$ was used. Growth rates are scaled using $\tilde{s}=s / \omega_{4}$ and $\omega_{A}^{2}=B^{2}(0) /\left[\rho q^{2}(a) R\right]$. Sote that the minor radjus, $a$, is set to 0.1 for this equilibrium. Thus, the value of $\eta$ used in the calculation for this case is a factor of 10 smaller than the inverse magnetic Reynold's number, $S^{-1}$. In order to analyze the stability of this equilibrium, the range of poloidal harmonics is fixed at $m \div-1,0, \ldots, j$ and the toroidal mode number was set at $n=1$. A tolal of $N_{s}=300$ radial points were used for the stability grid.

Figure 10 shows the harmonics of $\xi_{\psi}$ for the unstable tearing mode corresponding io $\eta=10^{-i}$. The resonant $m=2$ harmonic is cleariy dominant. Figure 11 gives the $\xi_{*}$ eigenfunction at $\eta=10^{-10}$. Figure $12 \mathrm{a}$ simply magnifies the vertical scale for the $\eta=10^{-10}$ eigenfunction in Fig. 11. This blowup of the eigenfunction causes most of the inner layer solution to be "clipped" off. The vertical scale of the blowup is normalized so that the maximum ralue that $\xi_{\psi}$ reaches (if not clipped off) is unity. Figure $12 \mathrm{~b}$ gives the $\xi_{\text {v }}$ function generated by the resistive PEST code. The PEST code calculates the eigenfunction only in the region away from the singular surface. For $\eta=10^{-10}$, one can expect good agreement with the asymptotic solution in the ideal region of the plasma. Figure 12c show's an overlay of Fig. 12a and Fig. 12b, which shows excellent agreement of the two codes.

Figure 13 demonstrates the $\eta^{3 / 5}$ growth rate scaling of the unstable tearing mode calculated by the NOYA-R code. The data shown in Fjg. 13 is given directly in Table 1:.

Table 12. NOVA-R Growth Rates versus Resistivity

$$
\begin{array}{ccccc}
\text { Resistivity } & 10^{-10} & 10^{-9} & 10^{-8} & 10^{-7} \\
\text { Growth Rate } & 9.20 \cdot 10^{-5} & 3.65 \cdot 10^{-4} & 1.37 \cdot 10^{-3} & 5.10 \cdot 10^{-3}
\end{array}
$$

The value of $\lambda^{\prime}$ represents the asymptotic behavior of the ideal solution very close to the resonant surface. Therefore, in order to measure $\Delta$ ' from the eigenfunction solution of the NOVA-f code. one must determine a region which is very close to the resonant surface and yet very far from the region where resistivity. inertia, and compressibility become important. This should be possible for sufficiently small resistivity $\left(\eta<10^{-10}\right)$ when the outer region equations become valid near the singular layer.

For the $\bar{m}=2, n=1$ resonant barmonic of $\xi_{w^{*}}=\vec{\xi} \cdot \nabla_{\varkappa}$. the following astmptotic 
coefficients were used to define $\Delta^{\prime}$.

$$
\xi_{\psi}^{(\bar{m})} / r \approx\left\{\begin{array}{ll}
C_{l}^{-} \frac{1}{\left|r-r_{s}\right|}+C_{s}^{-} & \text {for } r<r_{s} \\
C_{l}^{+} \frac{1}{\left|r-r_{s}\right|}+C_{s}^{+} \text {for } r>r_{s}
\end{array}\right\}
$$

where $r$ represents the square root of the normalized poloidal fux $(0 \leq r \leq 1)$. Here $r_{s}$ gives the location of the $m=2, n=J$ singular surface. $\Delta^{\prime}$ is then given by

$$
S^{\prime}=\frac{C_{1}^{-}}{C_{1}^{-}}+\frac{C_{3}^{+}}{C_{1}^{-}}
$$

Our procedure for determining the $C$ coefficients involves fitting the NOVA-R computed values of $\xi_{\psi}^{(\vec{m})} / r$ at successive pairs of adjacent grid points (near the rational surface) using the functional form given by Eqn. (6.1). Through analysis of the $\xi_{w}$, eigenfunction corresponding to $\eta=10^{-30}$. NOVA-R determined that $1.0 \leq \Delta^{\prime} \leq 1.4$. The resistive PEST result was $J^{\prime}=1.5 \pm .5$.

\section{Summary}

We have developed a formulation of the linearized resistive MHD eigenmode equations which prevents the computation of resistive modes from being degraded by spectral pollution from fast wave terms. This was accomplished by isolating the terms involved in the computation of the continuum spectra. The practical numerical advantages gained by using a formulation which separates out the continuum spectrum is the ability to accurately calculate resistive modes for the entire range of resistivities relevant to fusion raactors.

A derivation of the NOVA-R eigenmode equations has been presented. Straight field line magnetic thu coordinates are used, and this choice is well suited for efficiently approximating resunant modes with a limited range of poloidal harmonics. An explanation was given on how we expand into $M$ poloidal harmonics and numerically eliminate the first seven equations to form a system of $3 M$ second order differential equations. The NOVA-R code currently imposes that the tangential electric field and perpendicular displarement vanish at the wall. Expanding the code k handle different types of boundary conditions is straightforward.

In Sertion 11l. the following technical advantages of the NOVA-fi formtulation are printed out. 1.) It involves noly three coupled second order equations, in $\lambda=3$ variables. 2.) For finite resistjvity and for $\eta=0$, none of the variables have highest order derivatives (in $\frac{\partial}{\partial v}$ ) which are first derivatives. 3.) NOVA-R direrty recovers the identical results of a well established $2 \mathrm{D}$ ideal MHD code, NOVA, for $\eta=0$ and smonth connection exists hetween the finjte $\eta$ vOVA-R code and the NOY $A$ code in the $\eta \rightarrow 0$ linit. 4 ,) The SOVA-R equations do not become degenerate at any point in the plasma away from the magnetic axis. 
Section IV describes the numerical methods used in the NOVA-R code. In Appendix A we demonstrate how rewriting a B-Spline package 31-32 improsed the computational accuracy of numerical solutions by up to four orders of magnitude. The relative advantages offered by the NOVA-R cubic B-Spline package with respect to linear elements were demonstrated by examining an example problem. In order to study resistive modes. a nonuniform grid was used with many grid point placed near the resonant surface(s). Our approach and formulation for solving the resistive MHD equations has a straight forward extension from the cylindrical to the $2 \mathrm{D}$ problem.

In Section $V$, we have established the accuracy of the NOVA-RC code by comparing results with other established $1 \mathrm{D}$ codes. The NOVA-RC code recovered asymptotic matching results for resistivities significantly below the range of interest for fusion reactors and initial value results for resistivities well above the range of interest for fusion reactors. This demon:-trated that the NOVA-RC code is producing the most accurate results within the range of resistivities of interest for fusion reactors. Because cubic B-splines are being used. we are able to obtain converged results with an order of magnitude fewer grid points than linear finite elements would require. This advantage becomes very important for the $2 \mathrm{D}$ problem. The NOVA-RC results presented demonsirate the feasibility of extending our nun-asymptoric approach and choice of formulation to the $2 \mathrm{D}$ problem.

The NOYA-RC code is not limited by any sort of ordering assumptions or approximations that might limit the range of applicability. The wider range of applicability represents as important advantage over many of the previously developed $1 \mathrm{D}$ resistive $\mathrm{MHD}$ stability cudes. Because no ordering assumptions are made. our approach is more comprebensive in capability for the low resistivity regime than asymptotic matching codes. There is no reason to believe that the specific ordering assumptions used for the asymplotic approach will be comprehensive enough to include the behavior of all possible types of instabilities in the low resistivity lirnit. Other consistent orderings may exist. By avoiding any ordering schemes or assumptions, we are free to search for additional modes which mav not scale like the tearing or interchange modes in the low resistivity limit. In partirular. hoth the NOYA-RC and $2 D$ NOVA-R codes have found unstable modes which scale linearly with $\eta$. These modes are anticipated to be the abject of further research in the immediate fut ure.

The recovery of asymptotic matching results for magnetic Reynold's numbers as hight as $S=10^{30}$ validates our approach for removing spectral pollution by carefully choosing a formulation of the problem which "separates out" the continuum spectrunl. The accuracy of these low resistivity results represent a breakthrough for a non-asymp1otic code. The important problem of how to optimally choose a formulation for solving the resistive MHD stability equations has been resolved.

Sertion $\mathrm{VI}$ gives numerical results from the NOVA-R code. The ability of the NOVA-K code to properly analyze the stability of general axisymetrir equilibria in the icleal limit was demonstrated. The task of making this check has been greatly facilitated by chousing a formulation which performs the identical computation as the NOVA code in the idcal limit. The XOVA-R code was used to analyze the resistive stability of axisymmetric toroidal equilibria. A spheromak-like $2 \mathrm{D}$ equilibria which was unstable against resistive 
interchange modes was analyzed by the NOVA-R code. The NOVA-R code correctly recovered cylindrical results in the limit of infinite aspect ratio. An-equilibrium previously determined to be unstable to tearing modes by the resistive PEST code was also anaiyzed. We demonstrated the numerical ability of the NOVA-R code to resolve the unstable mode at $\eta=10^{-10}$. This justifies that the NOVA-R approach can make accurate calculations for low resistivities in the range relevant for fusion reactors. Excellent agreement with the PEST ralculated ejgenmodes and subsequent $A^{\prime}$ calculation was found.

The NOYA-R code provides quantitative information about what resistive MHD predicts, which may be compared with experimental results. In the future, the NOY. R stability analysis of specific equilibria can provide useful information relevant for the understancing and designing of future machines.

\section{ACKNOWLEDGEMENTS}

Significant contributions to the work were provided by the help of John Johnson and J. Manickam. I would also like to thank D. Monticello for many useful djscussions.

\section{Appendix A. The B-spline Package}

In this section we document the improvement in accuracy obtained by rewriting a B-Spline package obtained from the University of Texas at Austin |31-32\}. For an example problem, we illustrate the relative performance of linear finite elements, the Texas B-Spline package, and the package used by NOVA-R. For simplicity' only evenly spaced grids are used for the example problem.

Let $N$ represent the number of grid points used. For cubic B-Splines, one can converge tis the solution of a linear differential equation with an error that scales like $1 / \mathrm{A}^{\mathrm{t}}$. Linear elements converge like $] / N^{2}$. Although higher order elemnents converge still faster. BSplines are optimal for interpolating the coefficients of the differential equation. The coefficients of the differential equation are generally known only at the grid points, so an interpolation is required to define these coefficients eterywhere. Assume that one wishes to interpolate $V$ data points defined on the clomain, $0<x<1$. Among all functions, $f(x)$. which have continuous second derivatives and interpolate $N$ specified data points. the rubic B-Spline interpolation minimizes

$$
\int_{0}^{1} f^{\prime \prime}(x)^{-2} d x
$$

Interpolations made with higher order polynomials tend to oscillate between grid points 33 .

Regardless w what type of package is used, the ersors generated by differential ecguat ion solvers o:nerally stop converging for sufficiently large values of $N$. Let $\rightarrow$ represent the 
relative error caused by computer round off errors. For the Cray, $\Delta \approx 5 \cdot 10^{-16}$. Consider the differential equation,

$$
a(x) u^{\prime \prime}(x)+b(x) u(x)=0
$$

For the proper order of magnitudes, let $a(x) \sim a_{0}$ and $b(x) \sim b_{0}$. Consider finite elements with compact support over a domain having a length which varies as $\mathrm{J} / \boldsymbol{N}$. Regardless of what type of finite elements (or finite differences) are used, after discretizing the equation. $a(x) u^{\prime \prime}(x)-b(x) u(x)=0$, one will end up summing terms on the order of $a_{0} . V^{2}(1-\Delta)$ with $b_{0}$ terms. As $N$ becomes large, the numbers representing $b(x) u(x)$ start to get truncated. Thesefore, the accuracy of all second order differential equation solvers are limited by a "saturation" error on the order of $E R R \sim\left(a_{0} / b_{0}\right) N^{2} \Delta$.

The Texas package, however, will be shown to saturate with a worse error, which scales like $N^{3}$. For convenience, the Texas package defines all of the fourth order polynomials as an expansion about $x=0$. This creates a problem. In order to illustrate. let $x_{k}$ represent the value of $x$ at some $k^{\text {th }}$ grid point where $x_{k}$ is of oxder unity. Let $y=x-x_{k}$. In order to evaluate a cubis: B-Spline between $x_{k}$ and $x_{k \div 1}$, it is necessary to evaluate the highest order term.

$$
\phi(y)=a y^{3} \text { at } y=\delta, \text { for } \delta \sim 1 / N
$$

Accrunting for the roundoff error in $\delta$, the computer obtains $\phi(\delta)=a \delta^{3}(1+\lambda)^{3}$. But, if one uses $z$ instead of $y$ as the independent variable,

$$
\dot{\phi}(x)=\alpha x^{3}-3 x^{2} x_{k}+3 x x_{k}^{2}-x_{k j}^{3}
$$

For simplicity, assume that the computer only misrepresents the $x_{k}^{3}$ term by using $x_{k}^{3} \rightarrow$ $x_{k}^{3}(1-3)$. Now,

$$
\hat{\phi}(x)=\alpha\left(\delta^{3}+x_{k}^{3} \Delta\right) \sim \alpha \delta^{3}\left(1-C N^{3} \Delta\right) .
$$

By not defining the polynomials of the finite elements locally, the Texas package introduces a new sat uration error which scales like $N^{3}$. In the NOVA-R package. the cubic B-Splines polynomials are defined by expansions about the nearest grid points.

As previously stated, the performance of three different differential solving packages are to be compared. A simple differential equation problem is chosen.

$$
-u^{\prime \prime}(x)-x^{2} u(x)=\left[x^{2}-\left(\frac{\pi}{2}\right)^{2}\right] \sin \left(\frac{\pi x}{2}\right): u(0)=0 \text { and } u(1)=1 .
$$

The solution is

$$
u(x)=\sin (\pi x / 2)
$$

Le1 the computer generated solution be represented by $u^{*}(x)$. For $I$ evenly spaced grid prints given by $\left\{\boldsymbol{x}_{k}\right\}_{k=1}^{N}$. the error is defined to be

$$
\operatorname{ERR}(N)=\max _{\left.k \in i_{1}, N\right]}\left\{u^{*}\left(x_{k}\right)-\sin \left(\frac{\pi x_{k}}{2}\right)\right.
$$


The resulting errors, $E R R(N)$, are shown in Fig. 14.

Before encountering saturation due to round off errors, the Texas and NOYA-R packages converge like $1 / N^{4}$, while the linear elements converged like $1 / N^{2}$. As $N$ is increased. the Texas package prematurely saturates with an error given by,

$$
E R R \approx C N^{3} \triangle \text { for } \triangle=5 \cdot 10^{-16} \text { and } C=63 .
$$

The linear elements and the NOr:A-R package saturation error is given by.

$$
E R R \approx C N^{2} \Delta \text { for } \Delta=5 \cdot 10^{-16} \text { and } C=1.4
$$

For the minimal errors, the Texas B-Spline package obtained $E R R \approx 5 \cdot 10^{-9}, \mathrm{NOVA}-\mathrm{R}$ obtained $E R R \approx 5 \cdot 10^{-1 i}$, and linear elements obtained $E R R \approx 5 \cdot 10^{-9}$ with $N \approx 50$. $N \approx 150$ and $N \approx 2000$, respectively. For this example problem, the NOVA-R package represent.s a significant improvement rejative to both linear elements and the original Texas package.

\section{Appendix B. Grid Packing}

For problems with internal boundary lavers, the numerical convergence can be improved by employing a non-uniform grid which is relatively more dense in the layer. The reason grid packing algorithms tend to be somewhat complicated is that the length between adjacent regions should not be allowed to change too quickly. Numerical instabilities may arise if the separation between adjacent grid points changes too abruptly.

The NOVA-R code first calculates the value, $v_{s}$, where $q\left(\psi_{s}\right)=m / n$ for the resonant harmonic. An internal parameter, eps, is set to roughly the half width of the laver. Let $N$ represent the total number of $\psi$ grid points. For problems with only one rational surface, $N_{r}=N / \bar{t}$ grid points are packed into the resistive laver on a uniformly spaced grid. Outsile of the resistive laver the spacing between grid points grows geometrically. The spacing increases by a factor of $\left(1+p_{l}\right)$ to the left, where $p_{l}$ is among a set of discrete values which allow a grid point to exactly "land" at $\psi=0$. Similarly, the spacing increases by a factor of $\left(1-p_{r}\right)$ on the right, all the way to $\psi=1$. One needs to deternine $k$ i and $N_{r}$ the number of grid points to be used within the left and right regions oustide of the 2's - eps, z: - eps] interval. The values of $N_{l}$ and $N_{r}$ are determined by minimizing the statistical deviation of $p_{r}$ and $p_{l}$. subject to the constraint that $X=\lambda_{r}-Y_{i}-\lambda T$.

Generalizing this procedure for two resonant surfacs.a is done by defining two layers. $\imath_{s,}=$ eps and $2_{s 2} \pm$ cps. A point is chosen exactly in between the two layers in order to split the region between the lavers into two equal parts. The manner of aliocating grid porins: bеtween the four resulting "external" regions is again done by minimizing the statistical deviation of the four respective percentages. Here, the two middle regions of equal length should be constrained to have an equal number of grid points. 


\section{Bibliography.}

1. C. Z. CHENG AND M. S. CHANCE, J. Comput. Phys. 71, 124 (1987).

2. R. C. GRIMM, R. L. DEWAR, J. MANICKAM, S. C. JARDIN, A. H. GLASSER. ANI) M. S. CHANCE. (from Proc. $9^{\text {th }}$ Int. (onl. Battimore, 1982) Plasmu Physics and Controlled Vuclear Fusion Research 3, (1AEA, Vienna, 1983) p.35.

3. H. P. FlRTH. J. KILlEEN, AND II. N. ROSENBLUTh, Phys. Fluids 6. 4.59 (1963).

4. J. DELlCIA, S. C. JARDIN, AND A. H. GLASSER, Phys. Fluids 6, 1470 (1984).

5. A. H. GLASSER, J. M. GREENE, AND J. L. JOHNSON, Phys. Fluids 18, 87: (1975).

6. R. C. GRIMM, M. S. CHANCE, A. M. M. TODD, J. MANICKAM. M. OKABAYASHI, M. M. TANG, R. L. DEWAR, H. FISHMAN, S. L. MENDELSOHA. D. A. MONTICELLO, M. W. PHILLIPS, AND M. REUSCH, Nucl. Fusion 25, 805 (1985).

7. J. W. CONNOR, S. C. COWLEY, R. J. HASTIE, T. C. HENDER, A. HOOD. AND T. 1. M.A RTIN, Phys. Fluids 31, 577 (1988).

8. T. C. HENDER AND S. C. COWLEY, Phys. Fluids B 1, 2194 (1989).

9. M. S. CHU, M. S. CHANCE, J. N. GREENE, AND T. H. HEN-SEX, Phys. Fluids $B 297$, (1990).

10. J. A. DIBIASE AND J. KILlEEN, J. Comp. Phys. 24, 158 (1977).

11. D. D. SCHNaCK AND J. KllleEX, J. Comp. Phys. 34, 110 (1980).

12. R. IZZO, D. A. MONTICELLO, J. DELUCIA, W. PARK, AND C. M. RIT, Phys. Fluids 28, 903 (1985).

13. T. C. HENDER. R. J. HASTJE, AND D. C. ROBINSON. Nucl. Fusion 27, 1389 (1987).

I. L. A. CHARLTON, J. A. HOLMES. Y. E. LYNCH, AND B. A. CARRERAS. J. Comput. Phys. 86. 270 (1990).

I5. J. P. FREjisBERG AND D. W. HEWETT, J. rlasma Phys. 26. ITi (1981).

16. C. M. RI'L AND R. C. GRIMM, J. Plasma Physics 32. 207 (198-1). 
17. W. KERNER, K. LERBINGER, R. GRUBER, AND T. TSUNEMATSU. Comp. Phys. Comm. 36, 225 (1985).

18. C. Z. CHENG, L. CHEN, AND M. S. CH.ANCE. Ann. Phys. 161, 2] (1985).

19. K. APPERT, R. GRUBER, AND J. VACLAVIK. Phys. Fluids 17. 14i1 (197-t).

20. J. P. GOEDBLOD, Rijnhuizen Report No. 83-145. (1983).

21. M. D. KRUSKAL AND R. M. KULSRLD, Phys. Fluids 1, 265 (1958).

22. S. HAMADA, Progr. Theoret. Phys. 22. 145 (1959).

23. J. M. GREENE AND J. L. JOHNSON, Phys. Fluids 5, 510 (1962).

24. S. HAMADA, Nucl. Fusion 2, 23 (1962).

25. J. M. GREENE AND J. L. JOHNSON, Advances in Theoretical Physics 1. (Academic Press. New York, 1965) p.218.

26. J. L. JOHNSON, H. E. DALHED, J. M. GREENE, R. C. GRIMM. Y. Y. HSIEH. S. ( JARDIX, J. MAN!KAM, M. OKABAYASHJ, R. G. STORER, A. M. M. TODD. D. E. VOSS. AND K. E. WEIMER, J. Comp. Phys. 32, 212 (1979). (1980).

27. J. DELliCIA, S C. JARDIN, AND A. M. M. TODD, J. Comp. Phys. 37, 183

28. R. C. GRIMM, R. L. DEWAR, AND J. MANICKAM, J. Comp. Phys. 49, 94 (1983).

29. R. L. DEWAR, D. A. MONTICELLO. AND W. N.-C. SY, Phys. Fluids 27, Ii23 (1984).

30. GILBERT STRANG AND GEORGE J. FIX, An Analysis of the Finite Element Method. (Prentice-Hall, Englewood Cliffs, X. J., 1973) pp.119-120.

31. J. C. MILEY, The Lniversity of Texas Report FRCR No. 138. (197i).

32. J. C. WILE), The University of Texas Report FRCR Ao. 141. (1977).

33. C. DE BOOR. \& Practical Guide to Splines, (Springer-Yeriag. X. Y.. 1978) p.66 


\section{Figure Captions}

Figure 1. A comparison of the radial displacement of an unstable resistive isterchange mude, as computed by NOVA-RC and asymptotic matching. The NOYA-RC results for $\eta=10^{-7}, \eta=10^{-7}$ and $\eta=10^{-10}$ are given in (a). (c), and (e). The corresponding asymplotic results are given by (b), (d), and (f).

Figure 2. Convergence of the NOYA-RC $\eta=10^{-10}$ sesistive interchange mode growth rate as a function of the total number of grid points used on a grid with uniform spacing.

Figure 3. The NOVA-RC computed radial displacement of an unstable tearing mode for $\eta=10^{-4} \cdot \eta=10^{-7}$, and $\eta=10^{-10}$ is given in (a), (b), and (c) respectively.

Figure 4. The NOVA.RC computed unstable eigenmode for $\eta=3.5 \cdot 10^{-7}$ with $\sigma=0.0$. U.45. 0.48 , and 0.50 is gjven by $(a-b),(c-d),(e-f),(g-h)$, respectively. Blow-ups of the solution near the resistice layes are given by $(b),(d),(f)$, and $(h)$.

Figure 5. The NOHA-R computed harmonics of the radial displacement of an ideal MHD instability recovers the results published in Ref. i]!.

Figure 6. The flux surfaces of a nearly circular 2D spheromak-like equilibrium (with $a / K=.01)$. The equilibrium is unstabje to resistive interchange modes.

Figure 7. The convergence of the NOVA-R growth rate of the resistive interchange made for $a / R=.01$ and $\eta=10^{-7}$ is shown as a function of the number of radial grid points used in the calculation of the equilibrium. Some of the equilibrium quantities calculated begin to oscillate between grid points at high $N_{c}$, confirming the degradation away from $1 / N_{c}^{2}$ convergence shown for high $N_{e}$.

Figure 8. The NOVA-K computed radial displacement of the unstable resistive interchange mode at $\eta=10^{-\hat{i}}$.

Figure 9. Comparison of NOVA-R growth rate results at $a / R=.01$ with the NOVA-RC' compused cylindrical limjt.

Figure 10. The NOVA-K computed eigenmode of a unstable resistive tearing mode at $\eta=301^{\top}$.

Figure 11. The NOYA-K computed eigenmude of a unstable resiative tearing mode al $\tau_{I}=10^{-10}$.

Figure 12. A vertical scale blow-up of the same data scen in Fig. 11 is givey by (a). This is to be compared with the ejgenmode computed by the resistive PEST code. given in (b). 
An overlay of (a) and (b) is given in (c), showing excellent agreement.

Figure 13. Scaling of the growth rate with resistivity for the unstable tearing foode.

Figure 14. A comparison of the numerical errors for three different finjte element packages demonstrates the superior accurary of the modifted package used by the NOYA-R and NOYA-RC codes. 
Figure 1a NOVA-RC $\eta=10^{-4}$

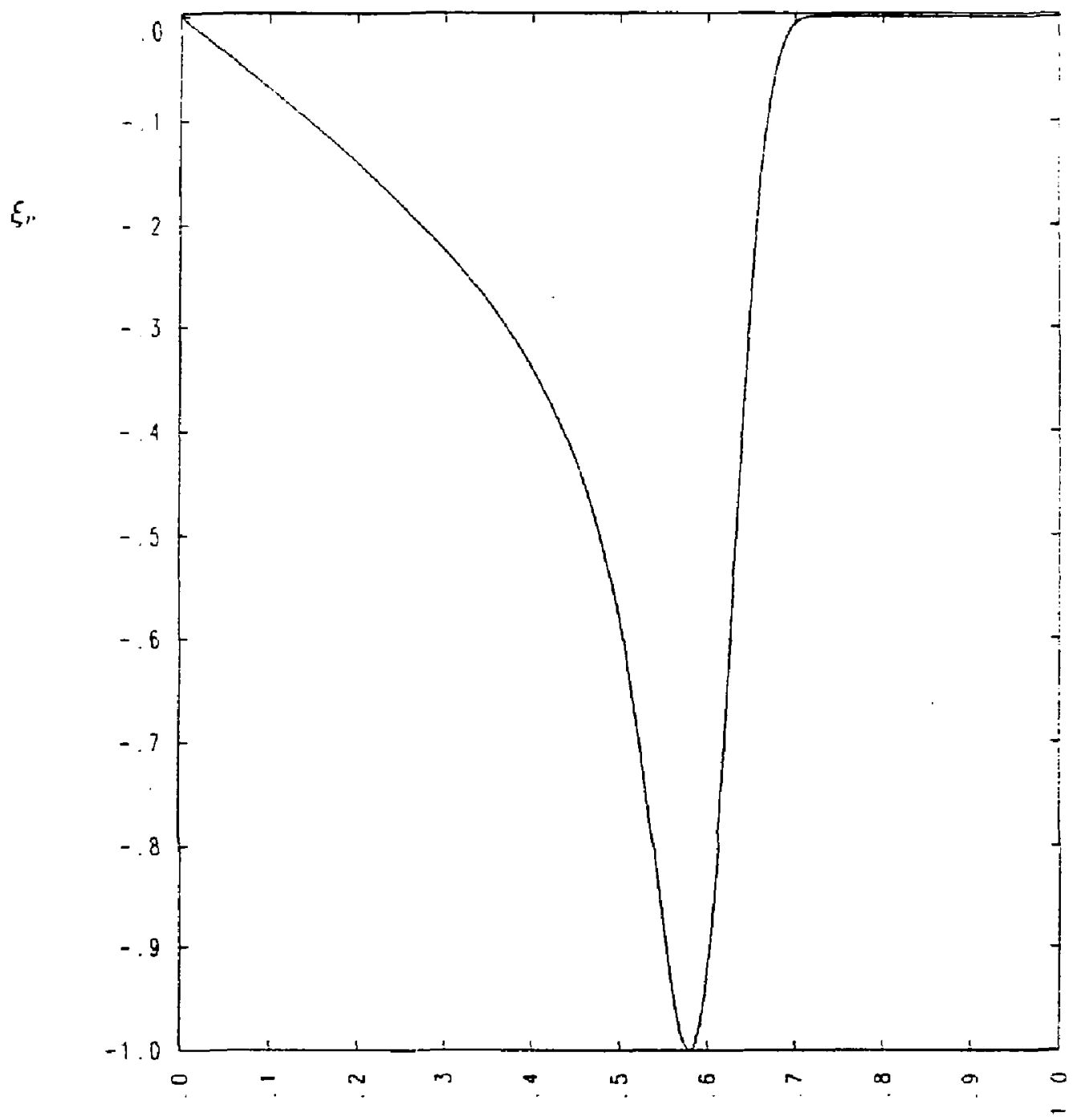

MIINOR RADIUS

F1g. 1 
Figure $1 \mathrm{~b}$ Asymptotic Natching $\eta=10^{-4}$

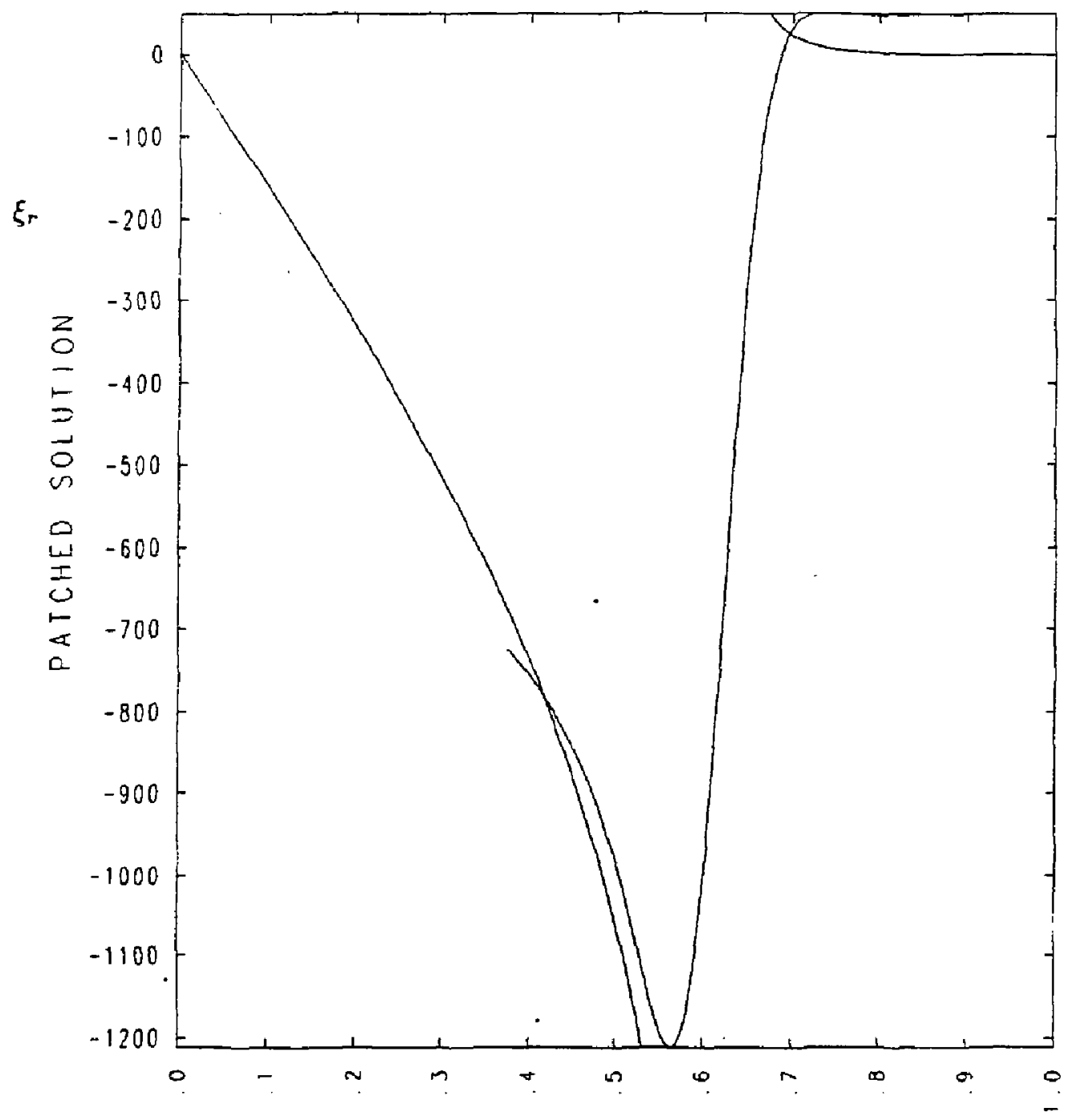

MINOOR RADILS

Fig. 2 
Figure 1c NOVA-RC $\eta=10^{-\bar{\tau}}$

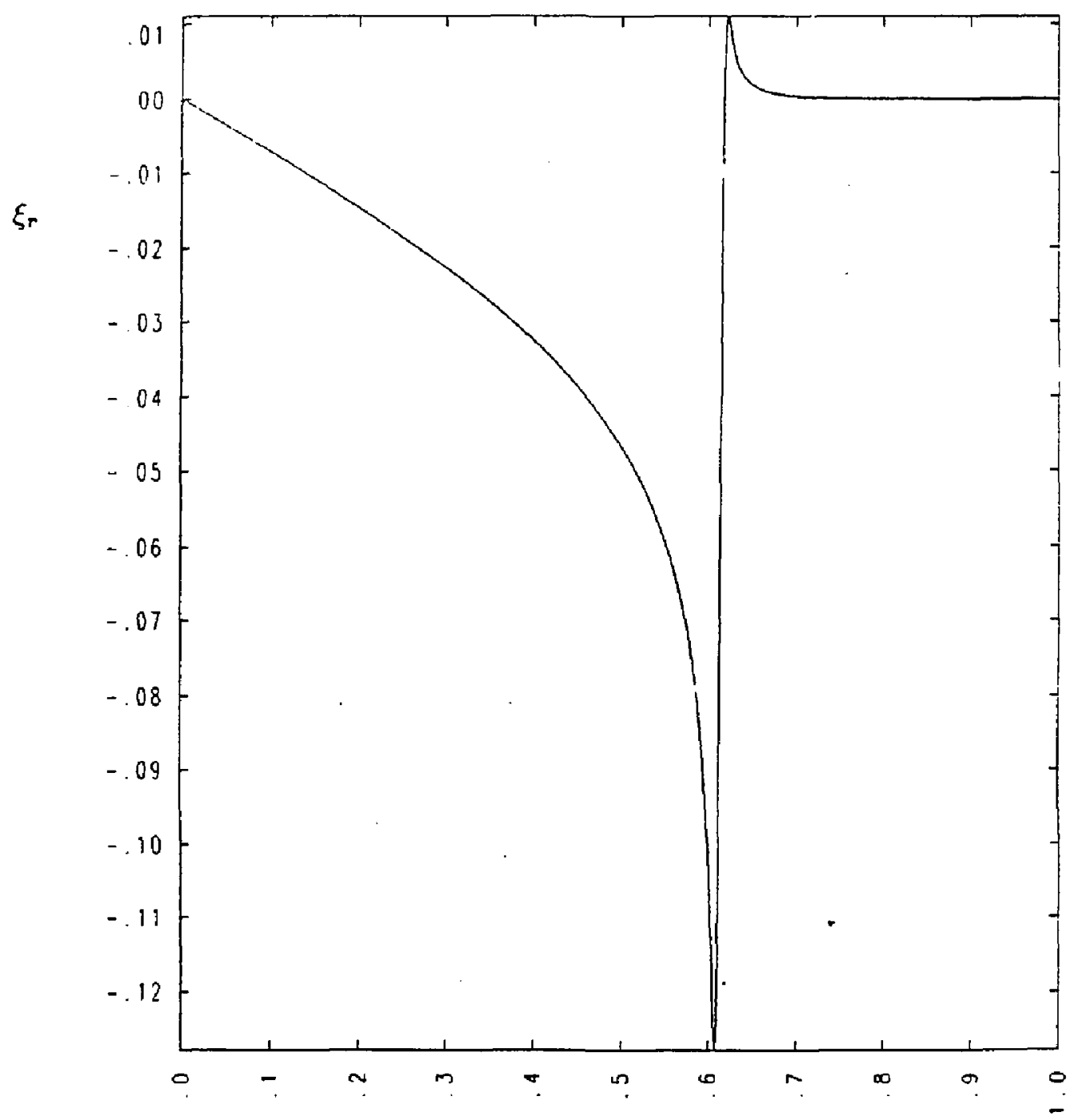

MINOR RADIUS

F1g. 3 
Figure 1d Asymptotic Natching $\eta=10^{-7}$

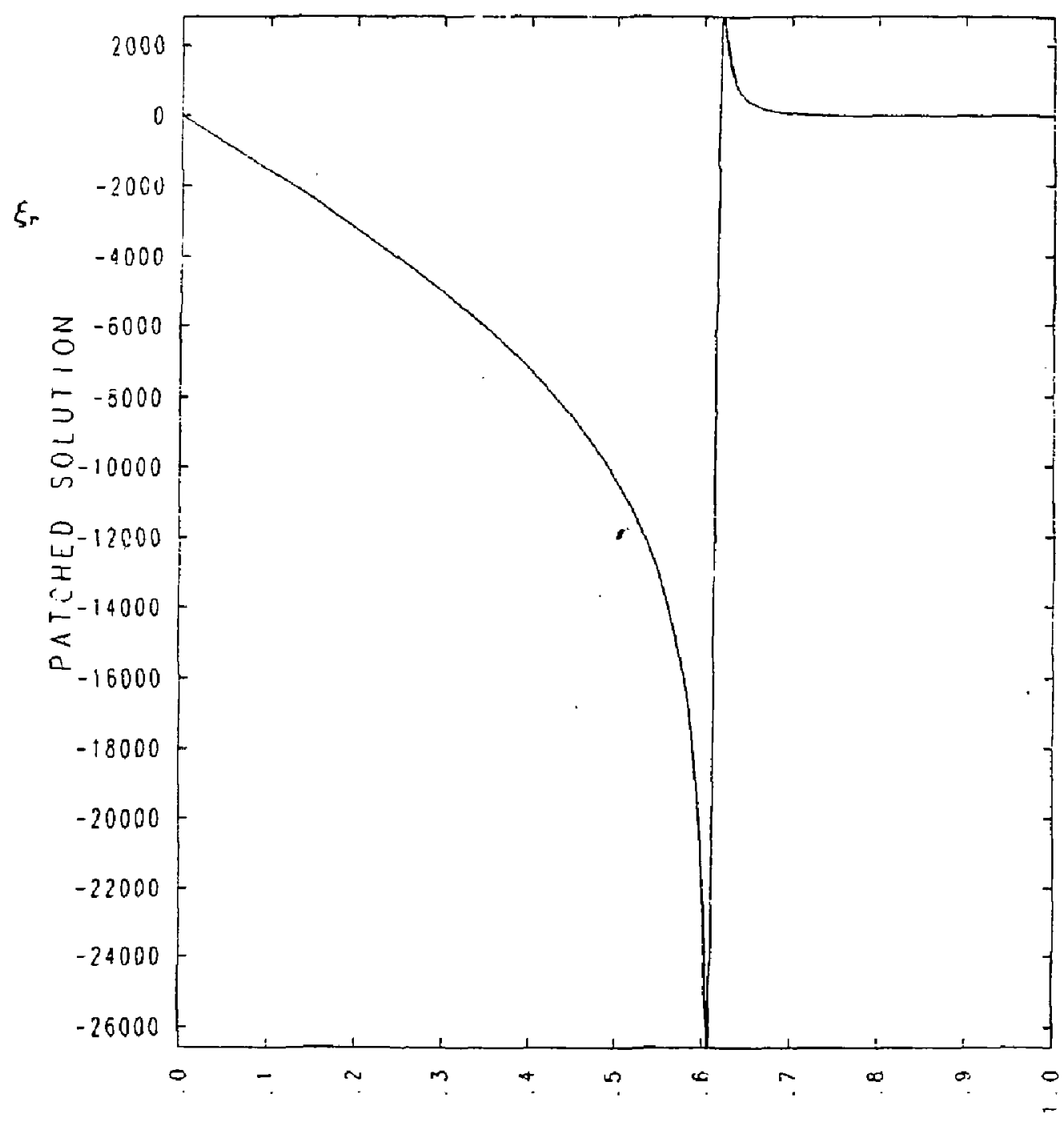

ININOR RADIT'S

F1g. 4 
Figure le NolA-RC $\eta=10^{-10}$

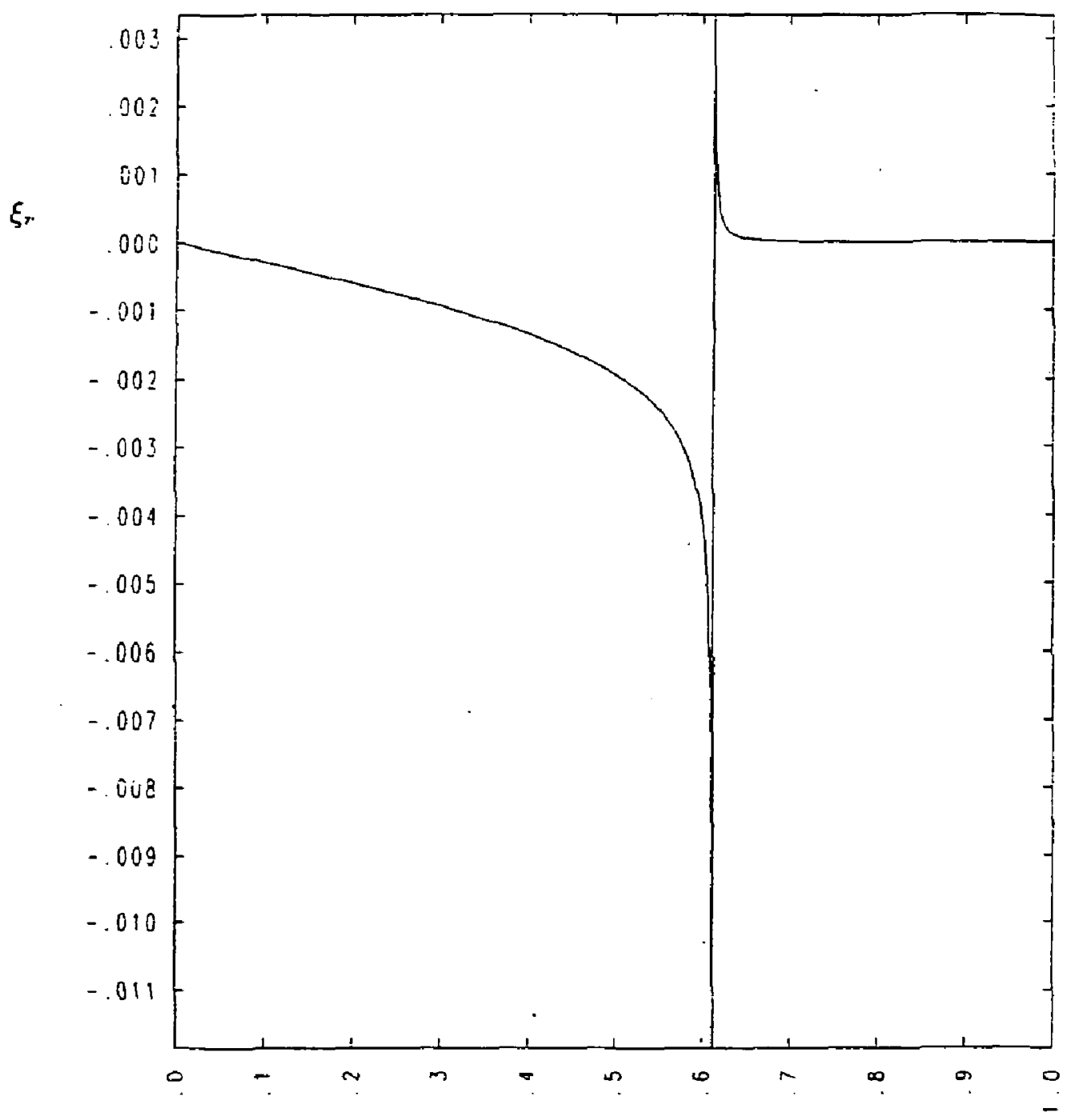

MIIOR RADILS

Fig. 5 
Figure 1f Asymptotic Matching $\eta=10^{-10}$

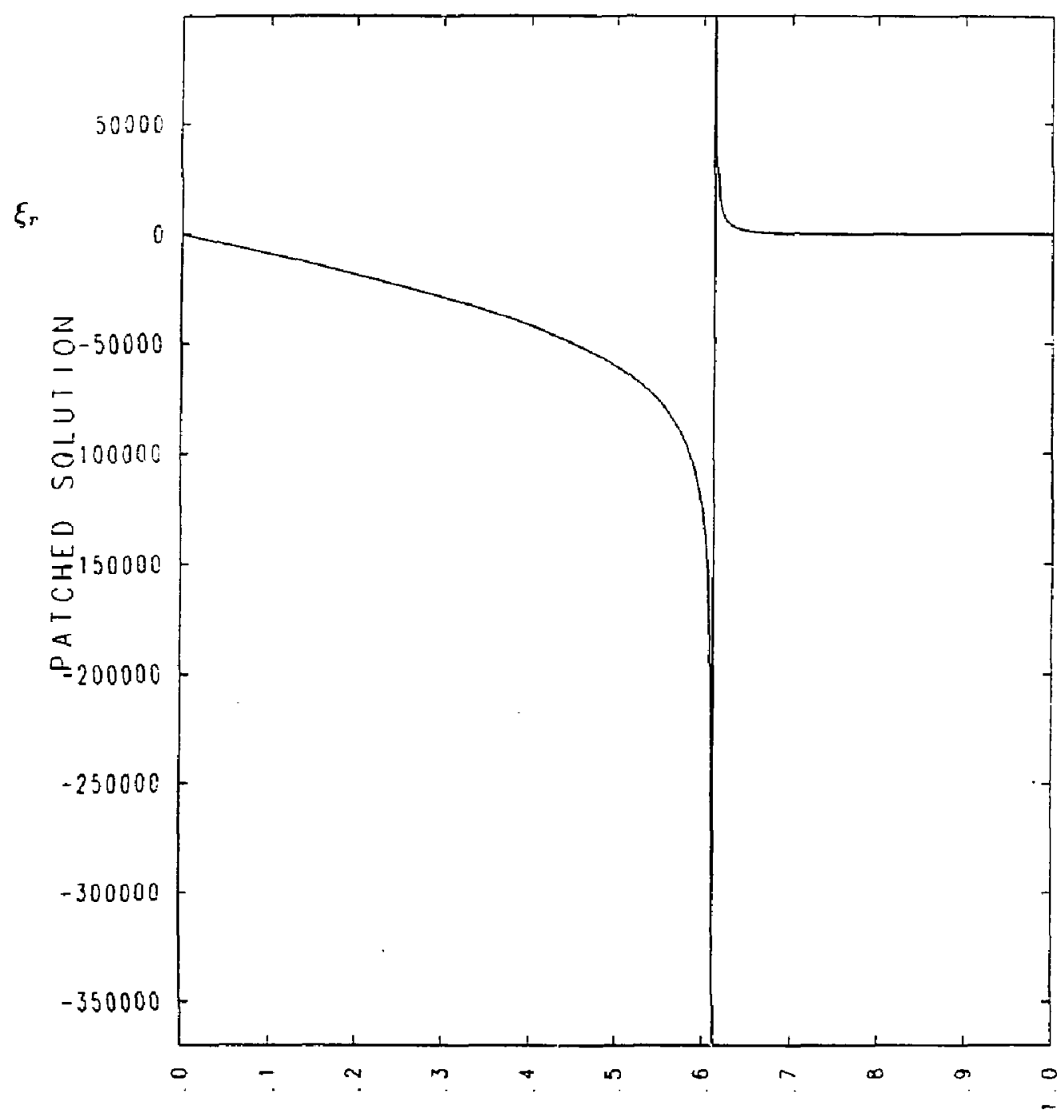

MINOR RADIUS

Fig. 6 
Figure 2 Convergence of Growth Rate with a Uniform Grid

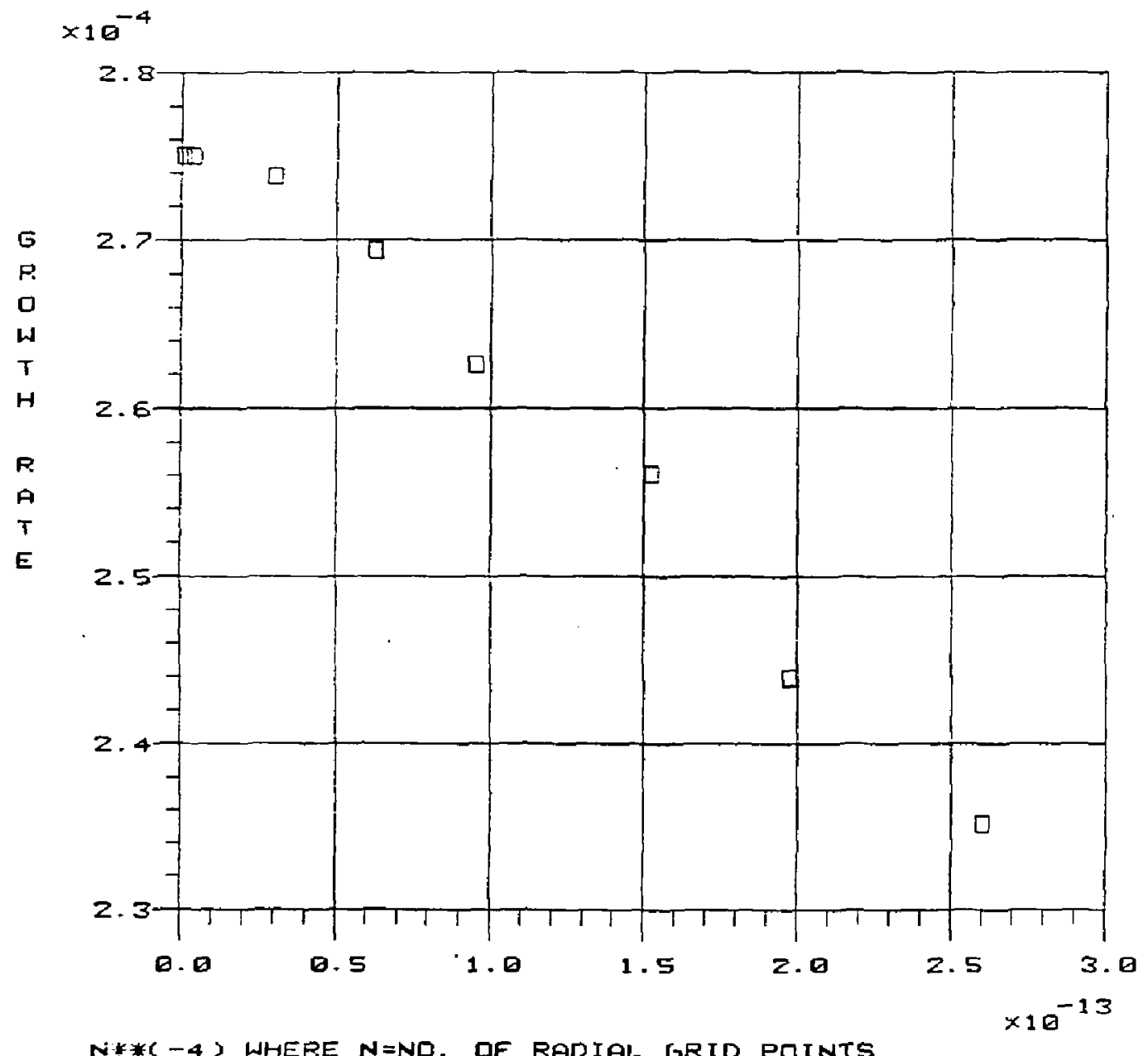

Fig, 7 
Figure 3a NOtA-RC $\eta=10^{-4}$

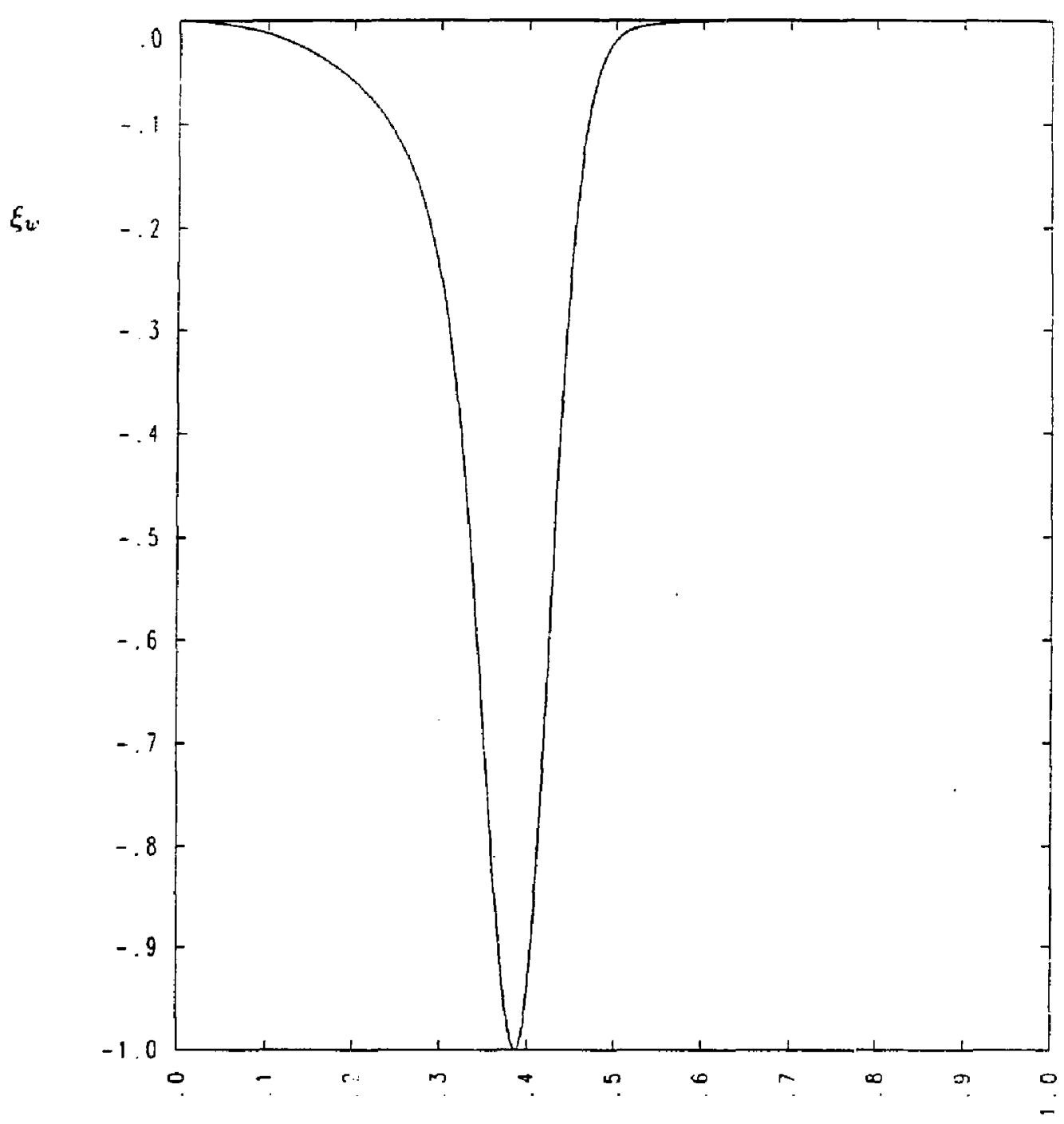

\section{MINOR RADIUS}

F1g. 8 
Figure $3 \mathrm{~b} \quad$ NOrA-RC $\eta=10^{-7}$

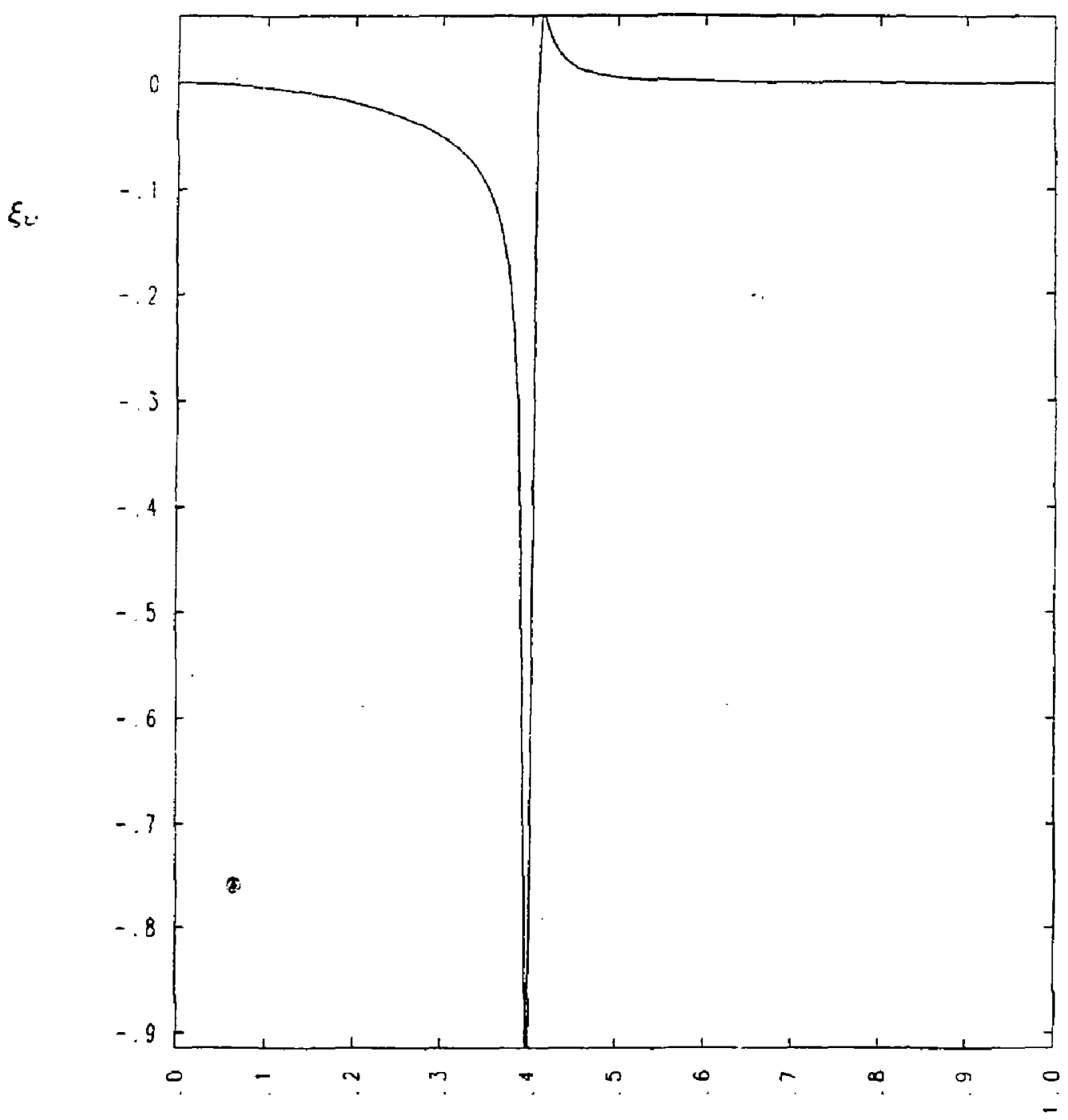

MINOR RADIUS

Fig. 9 
Figure 3c NOVA-RC $\eta=10^{-10}$

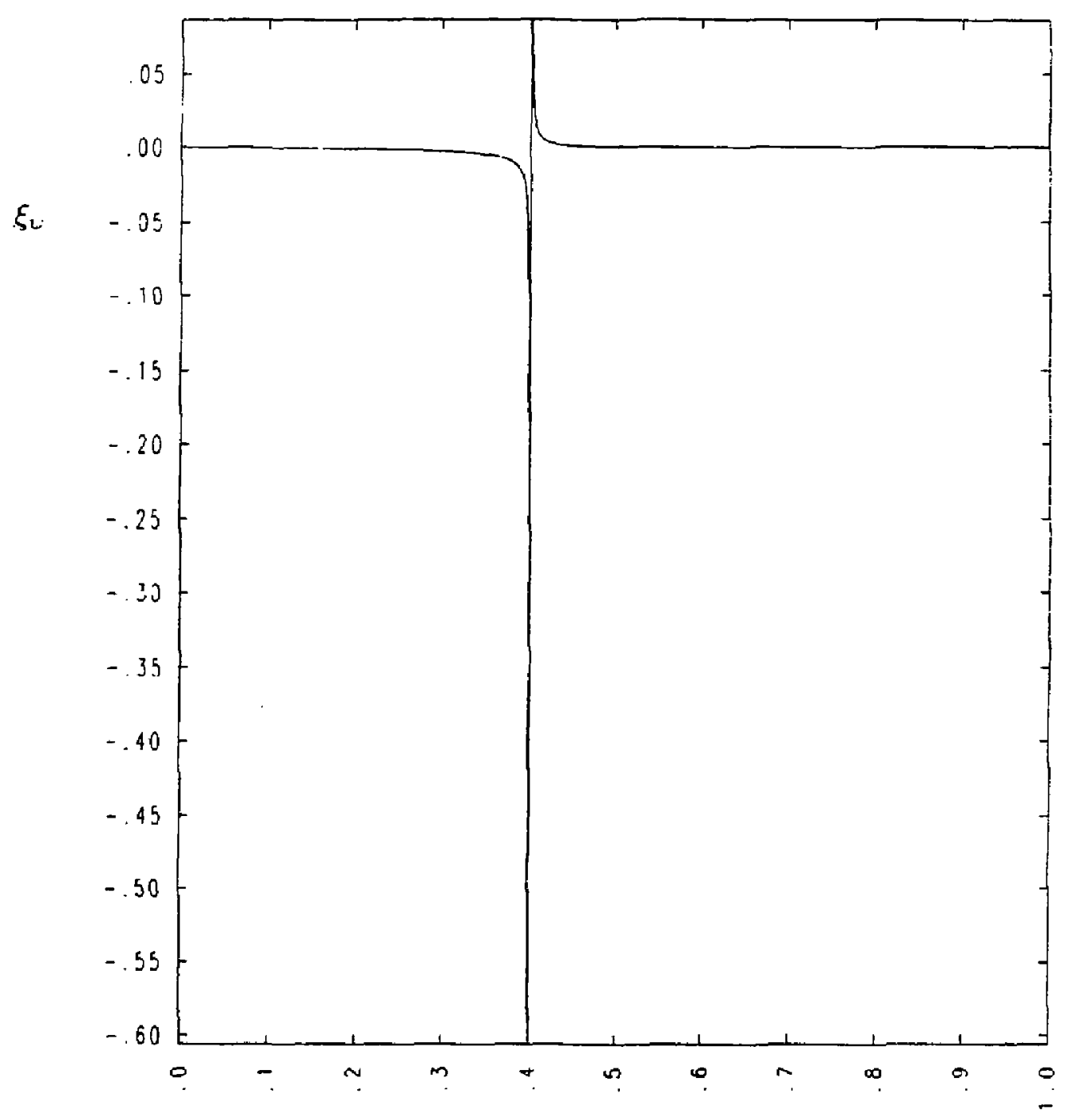

MINOR RADIUS

F18. 10 
Figure ta NOVA.RC $a=0$

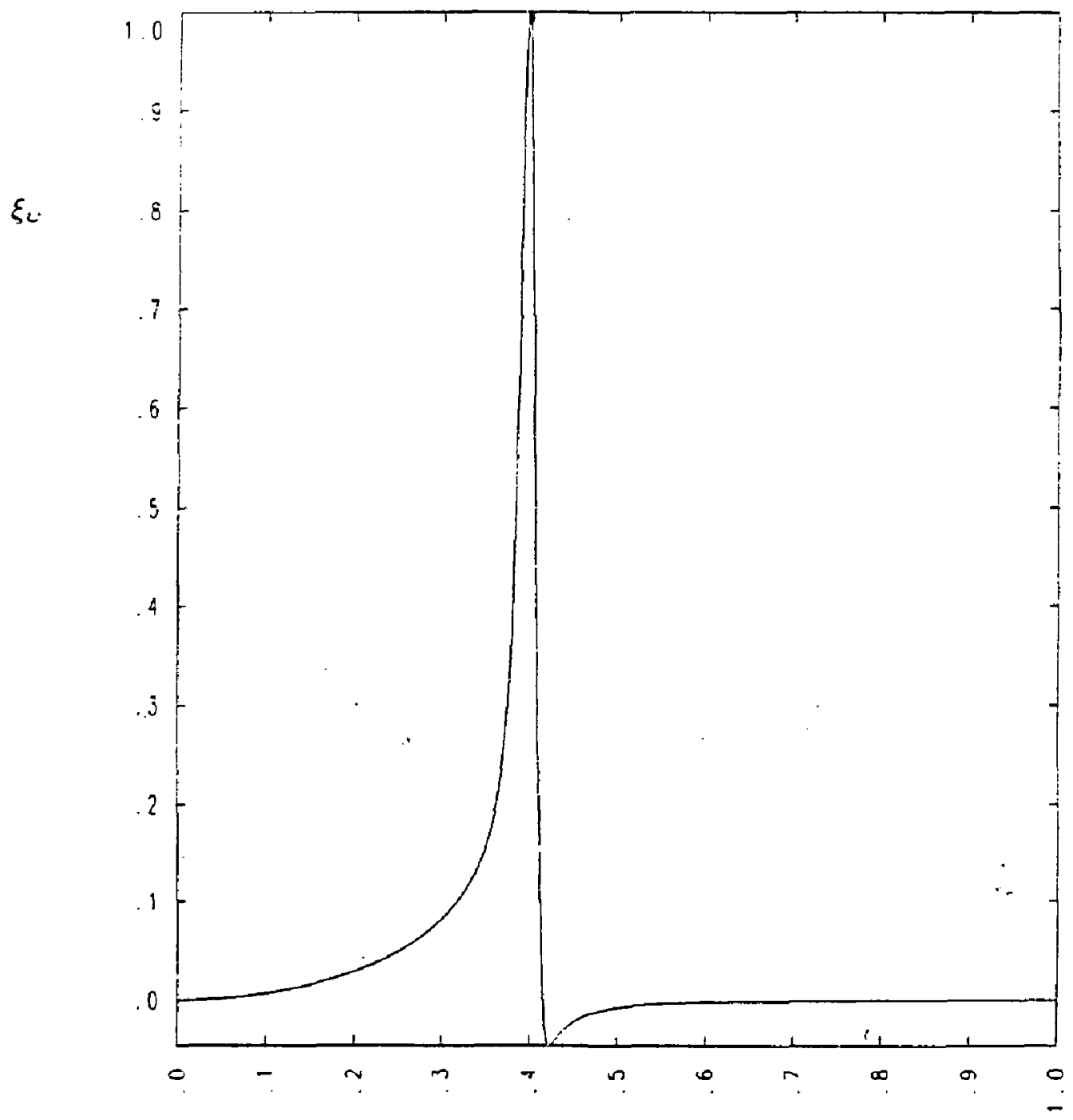

MINOR RADITS

F18. 11 
Figure $4 \mathrm{~b}$ NOTA-RC $\alpha=0$ Blowup of the Resistive Layer

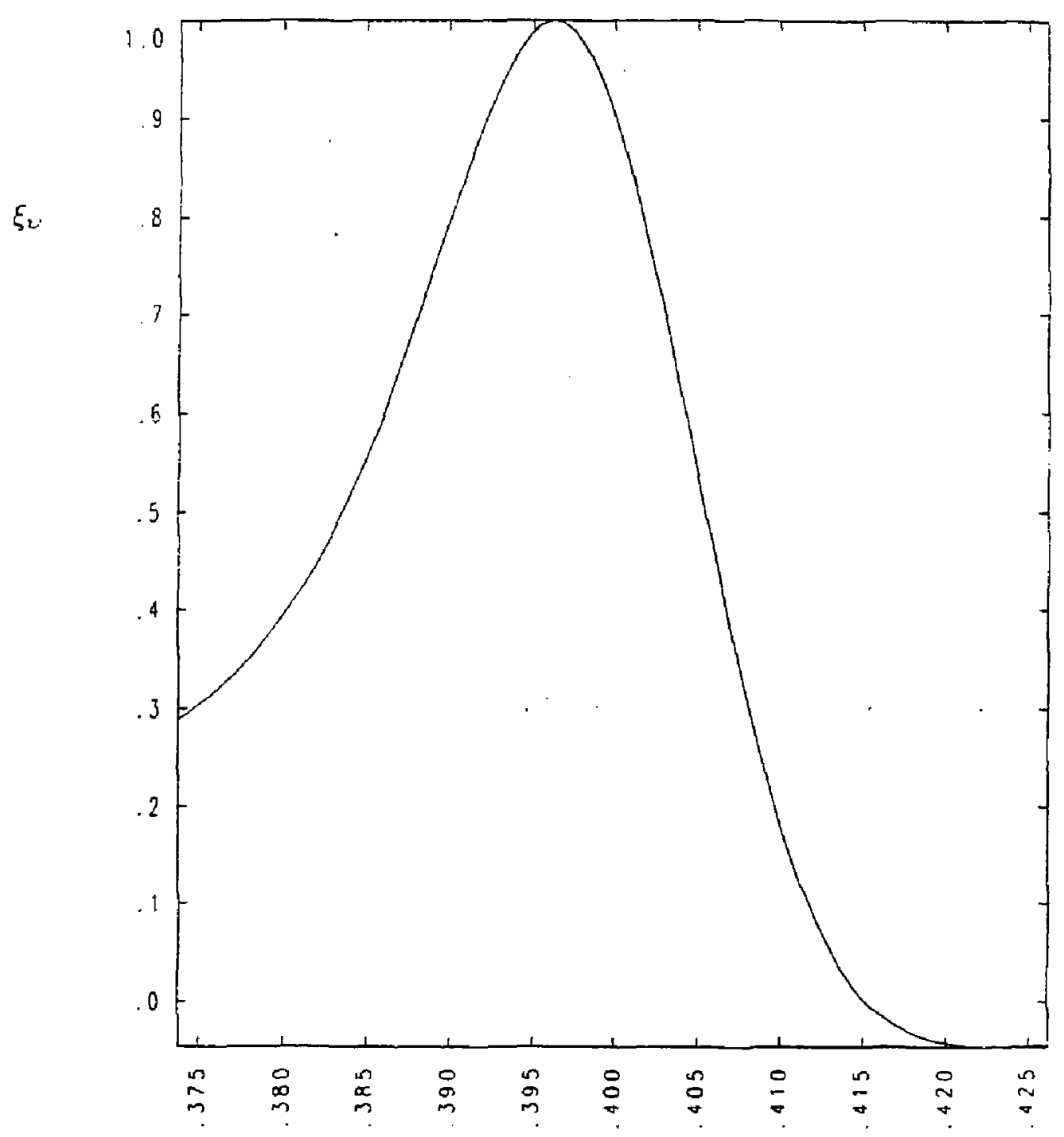

MIYOR RADIUS

Fig. I 2 
Figure Ac NOVA-RC $\alpha=0.45$

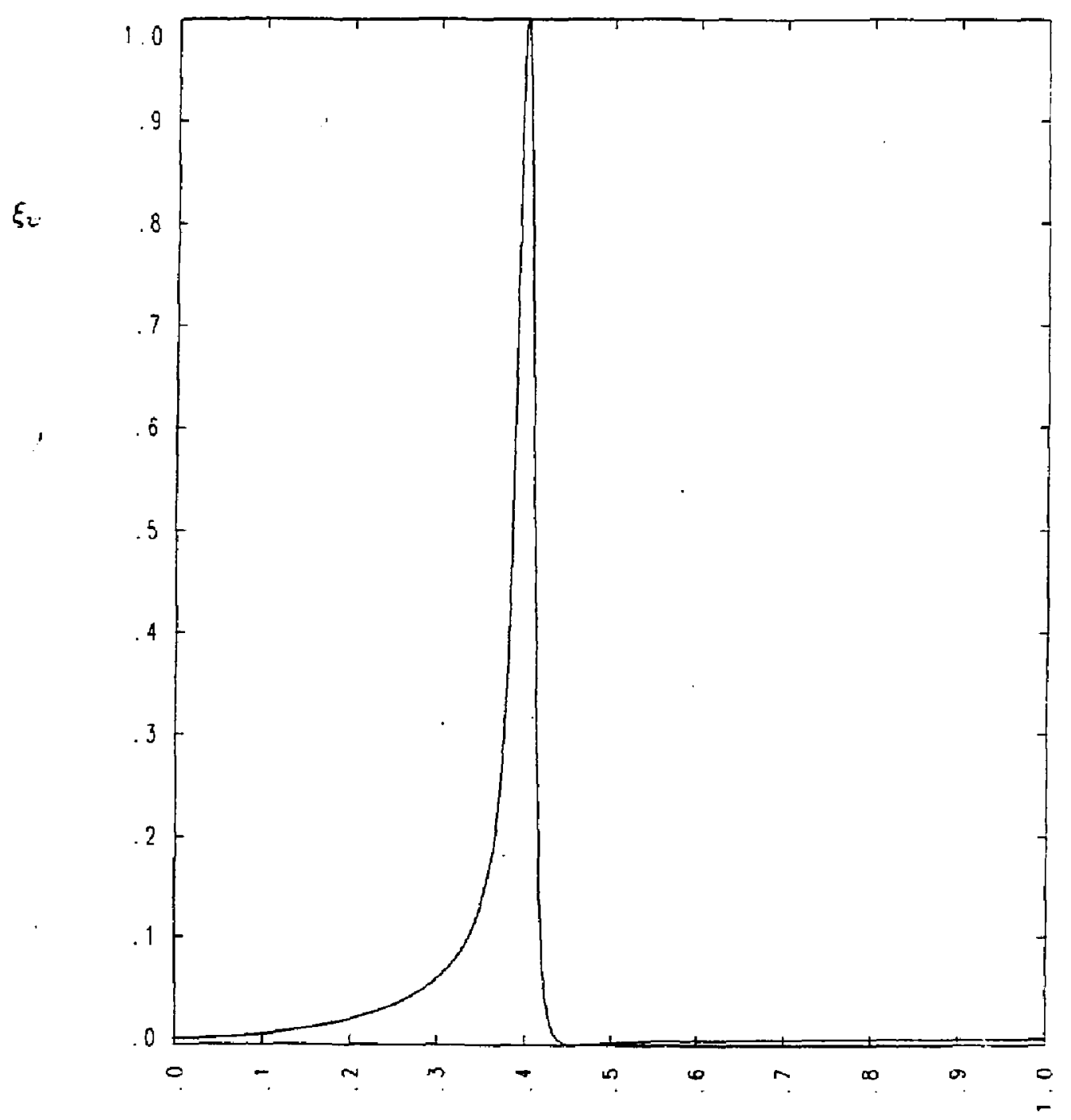

MINOR RADIUS

FIg, 13

48 
Figure 4d NOVA-RC $\alpha=0.45$ Blowup of the Resistive Layer

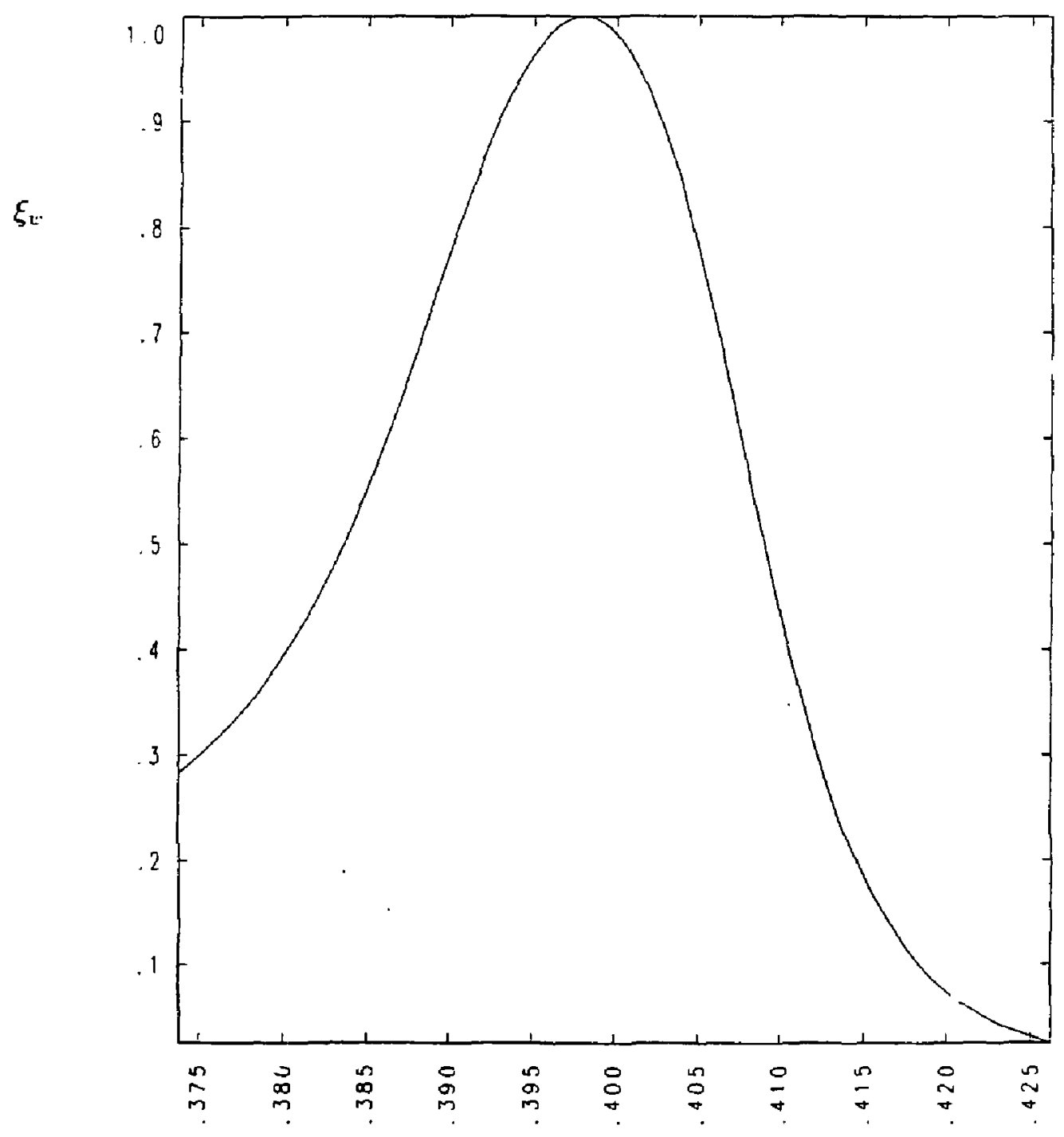

MINOR R.ADIUS

F1g, 14 
Figure $4 \mathrm{e}$ NOVA-RC $\alpha=0.48$

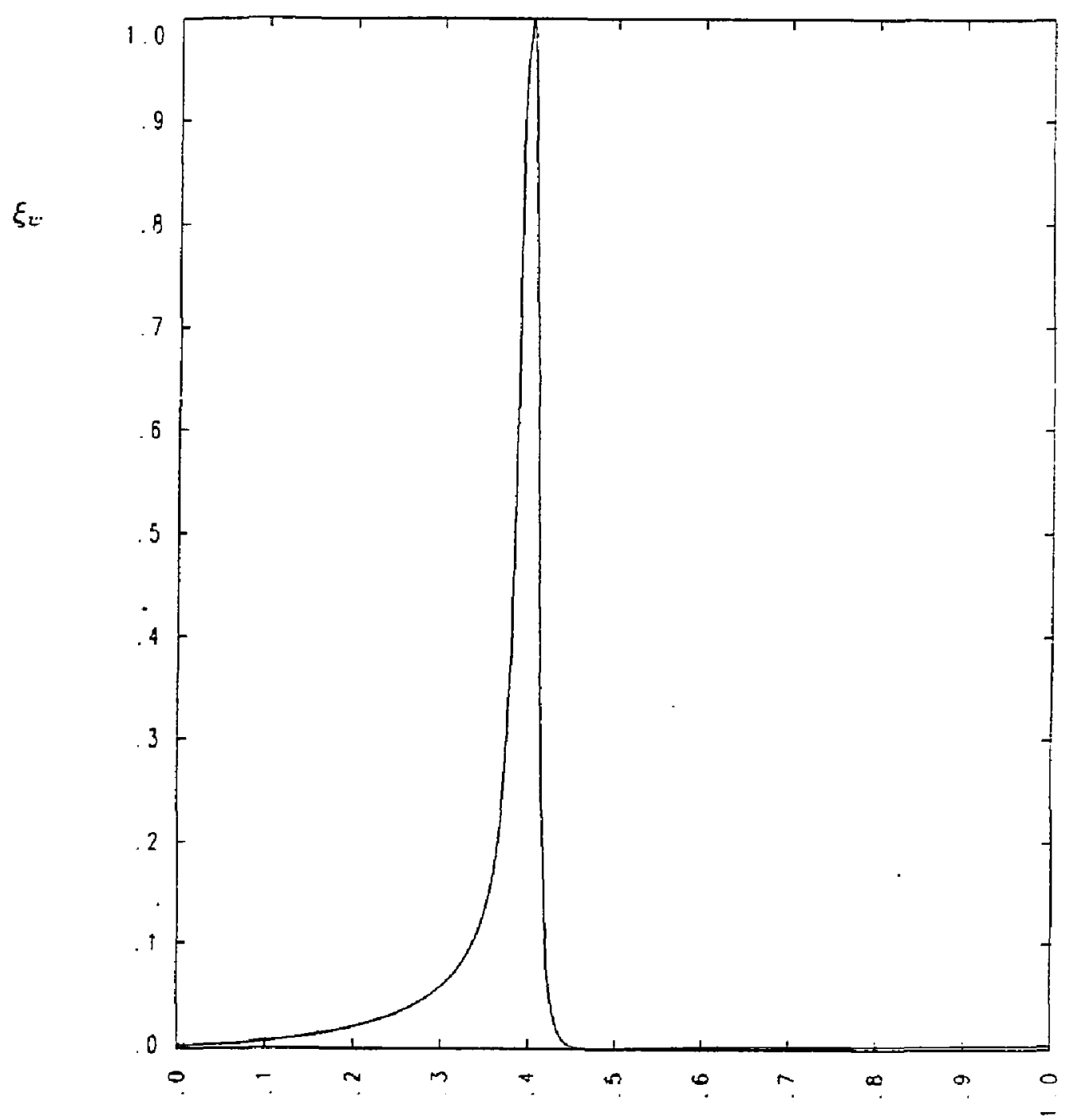

MINOR RADIUS

Fig. 15 
Figure 4f NOVA-RC $\alpha=0.48$ Blowup of the Resistive Layer

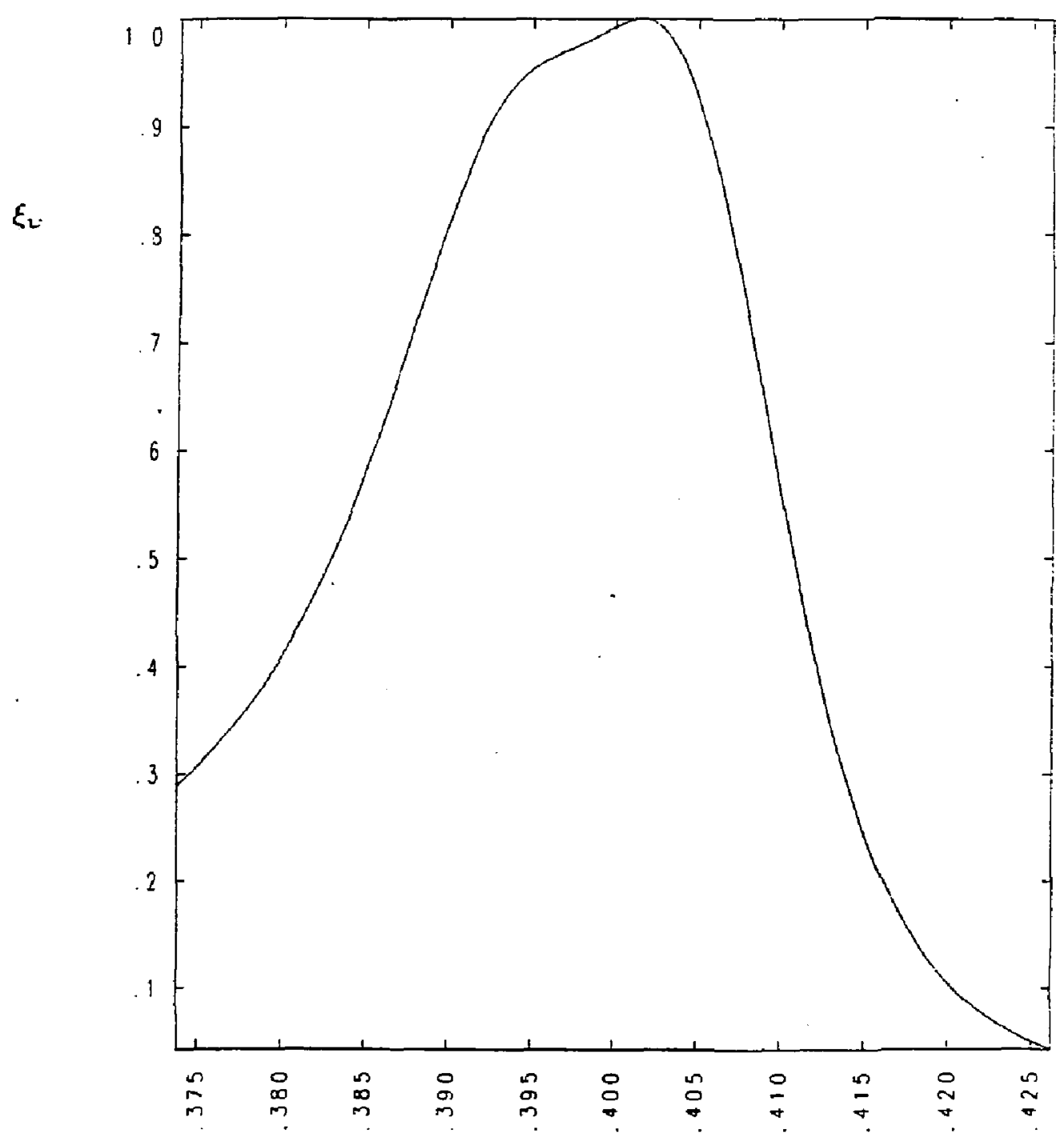

\section{MIINOR RADIUS}

Fig. 16 
Figure $4 \mathrm{~g} \quad$ NOVA-RC $\alpha=0.5$

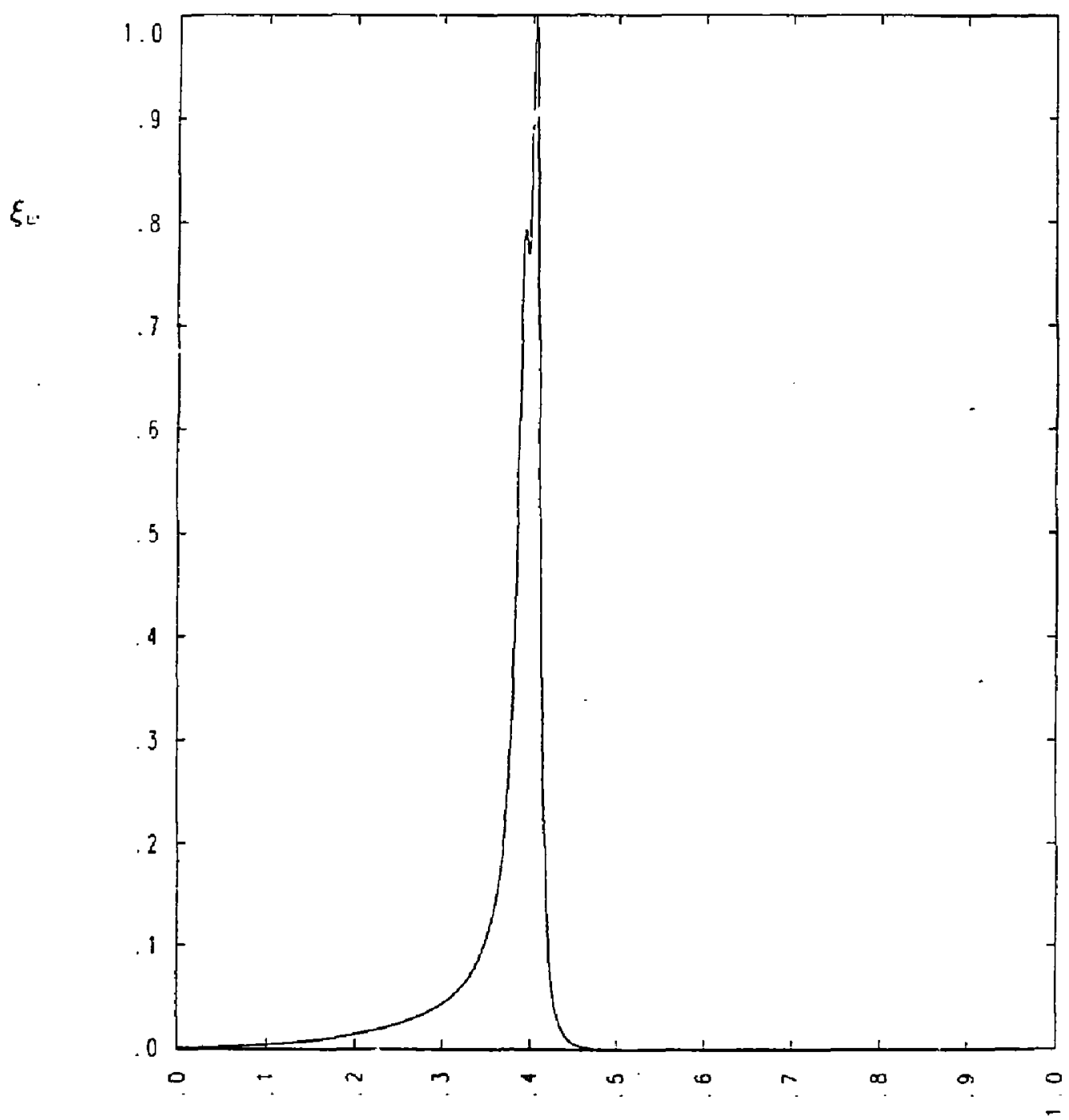

MIINOR RADIUS

F1g. 13 
Figure 4h NOVA-RC $a=0 . \overline{5}$ Blowup of the Resistive Layer

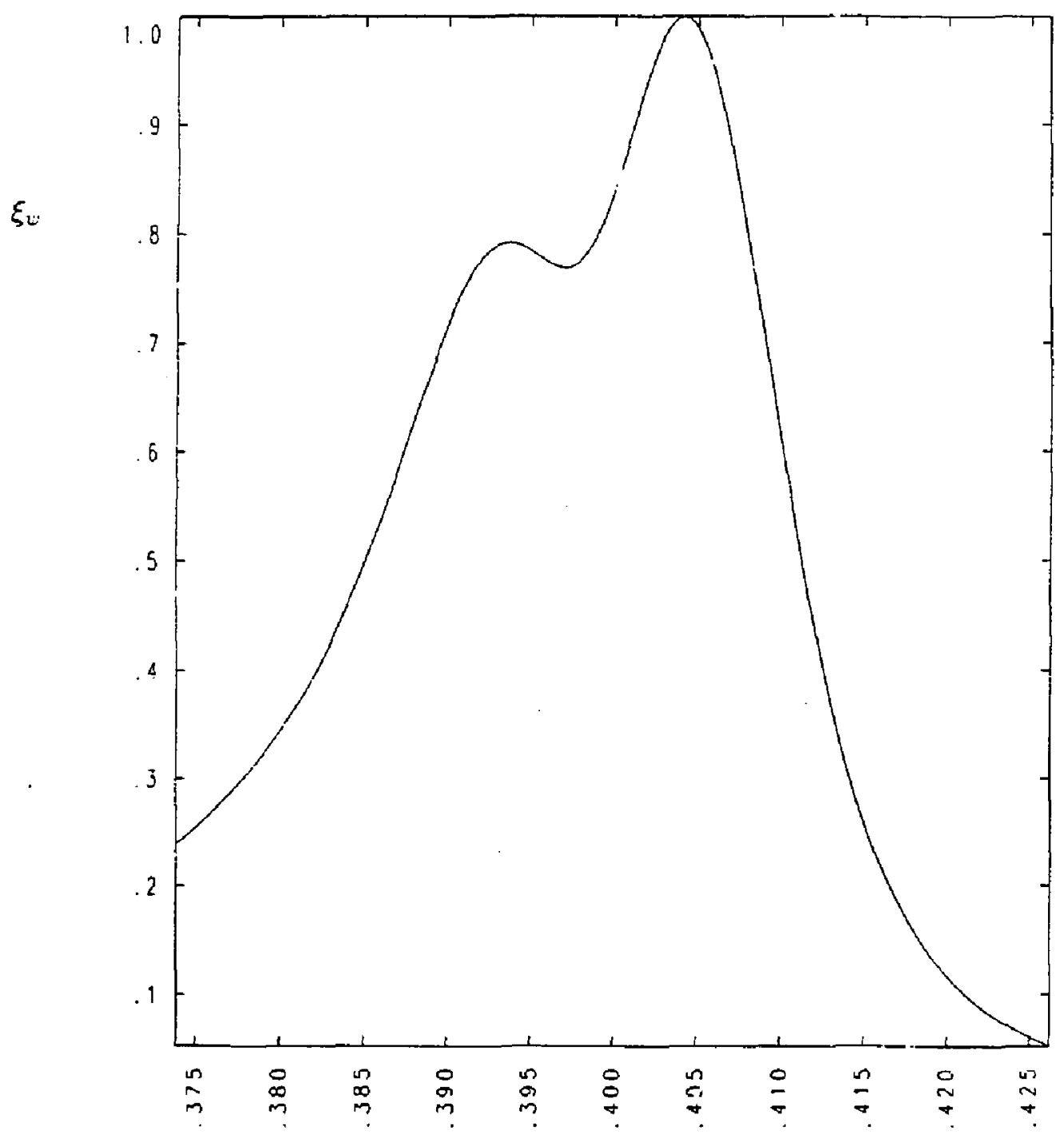

MINOR RADICS

F1g. 18 
Figure 5 NOTA-R $\eta=0$ Ideal Instability

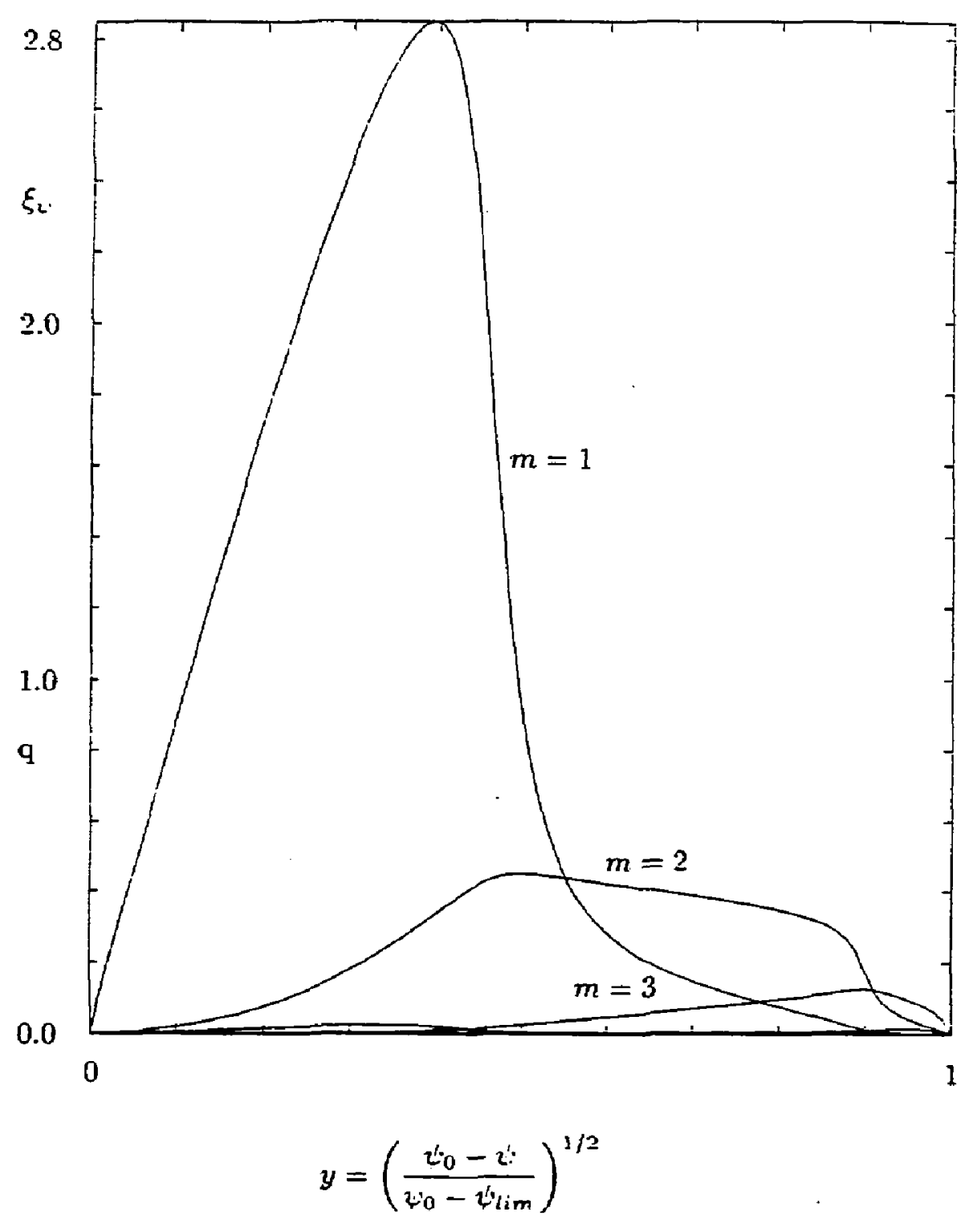

Fig. 19 
Figure 6 Spheromak 2d Equilibrium

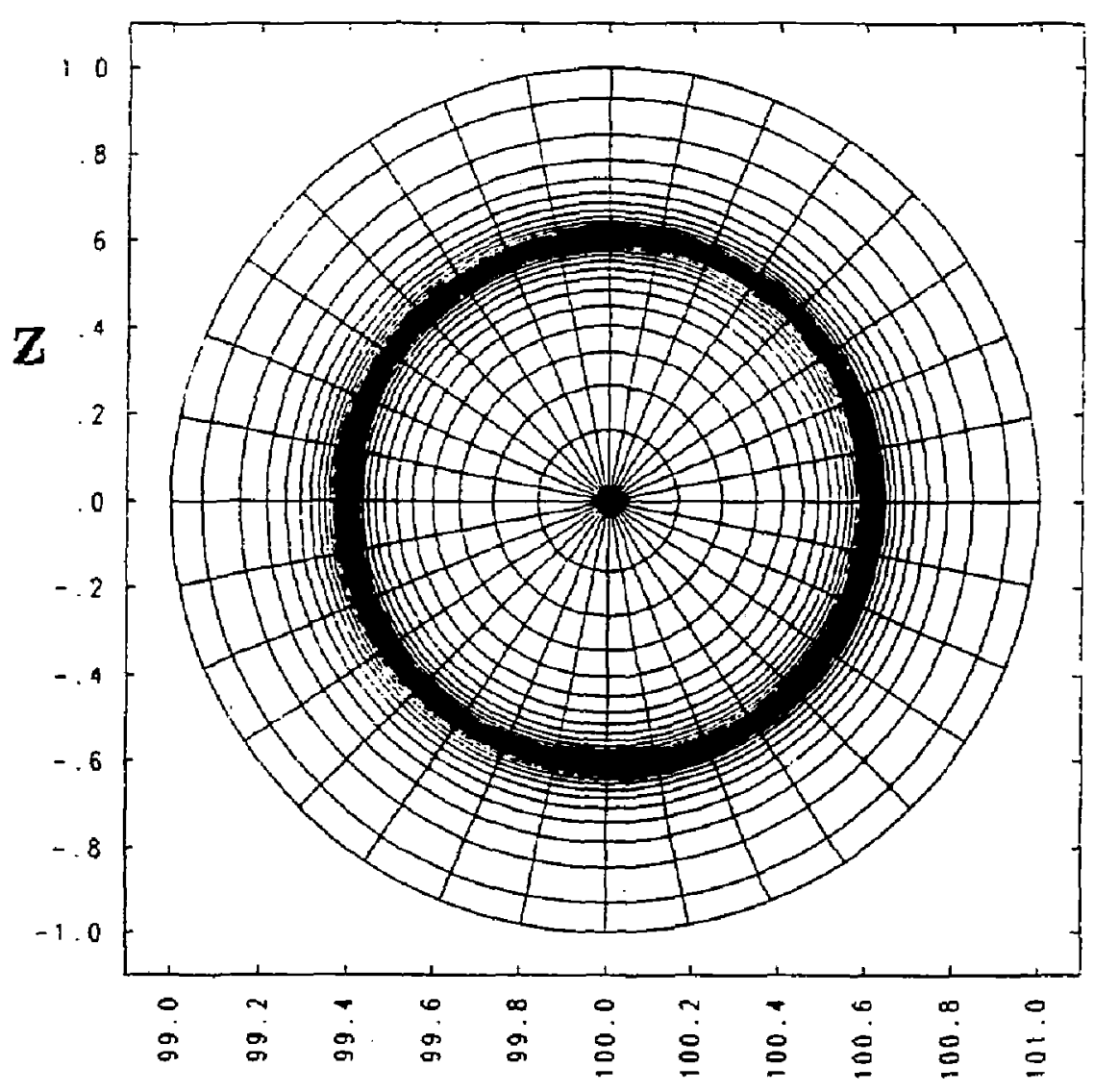

$22: i 0: 545$

$01 / 11 / 90$

P P P L

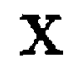

F1g. 20 
Figure 7 Convergence of NOVA-R Growth Rate at $\eta=10^{-i}$

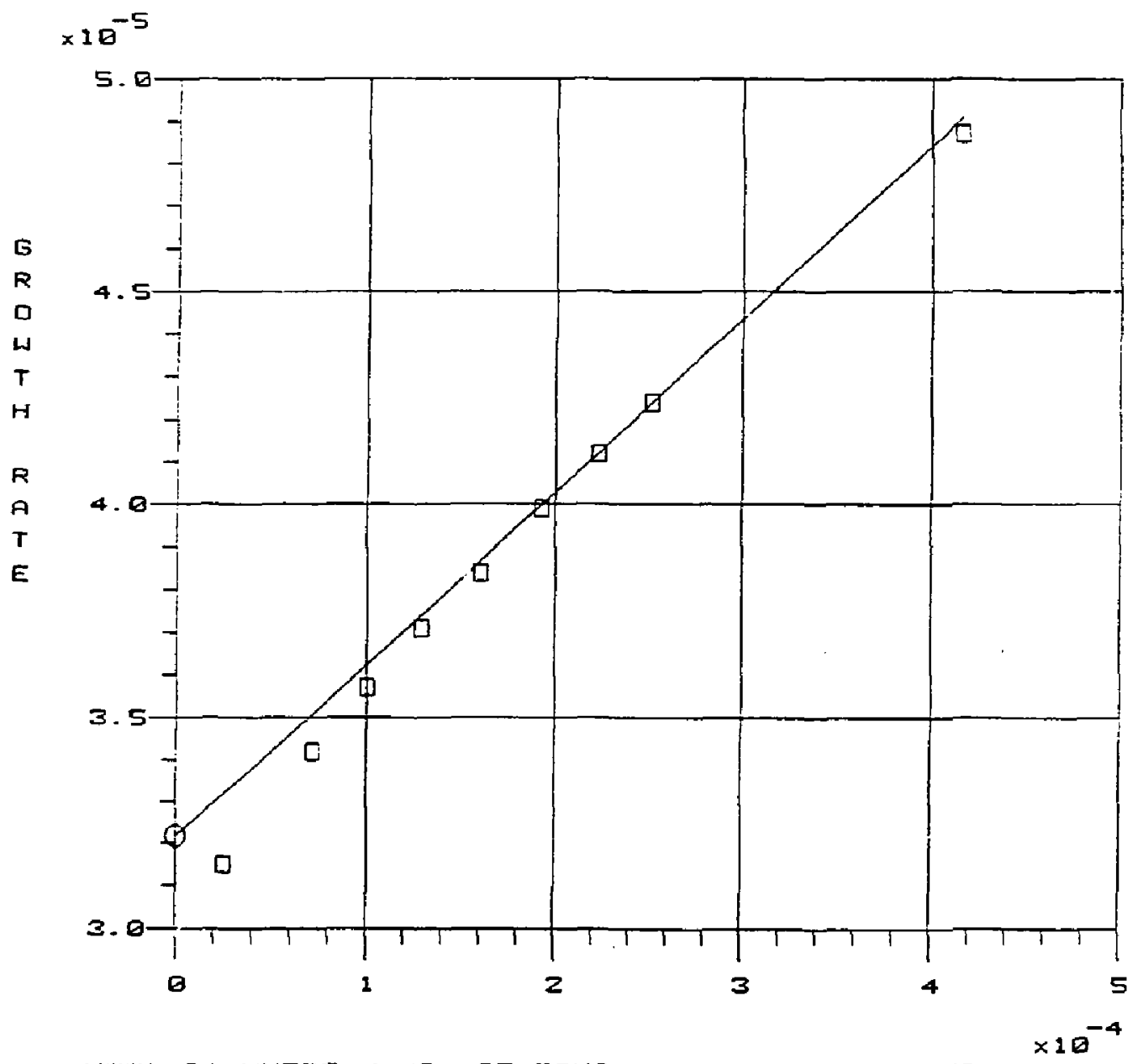

N**(-2) WHERE N=NO. OF EOUILIGRIUM RADIAL. GRID POINTS

F1g. 21 
Figure 8 NOVA-R Resistive Interchange Instability-

$$
N_{e}^{v}=200 \text { and } \eta=10^{-7}
$$

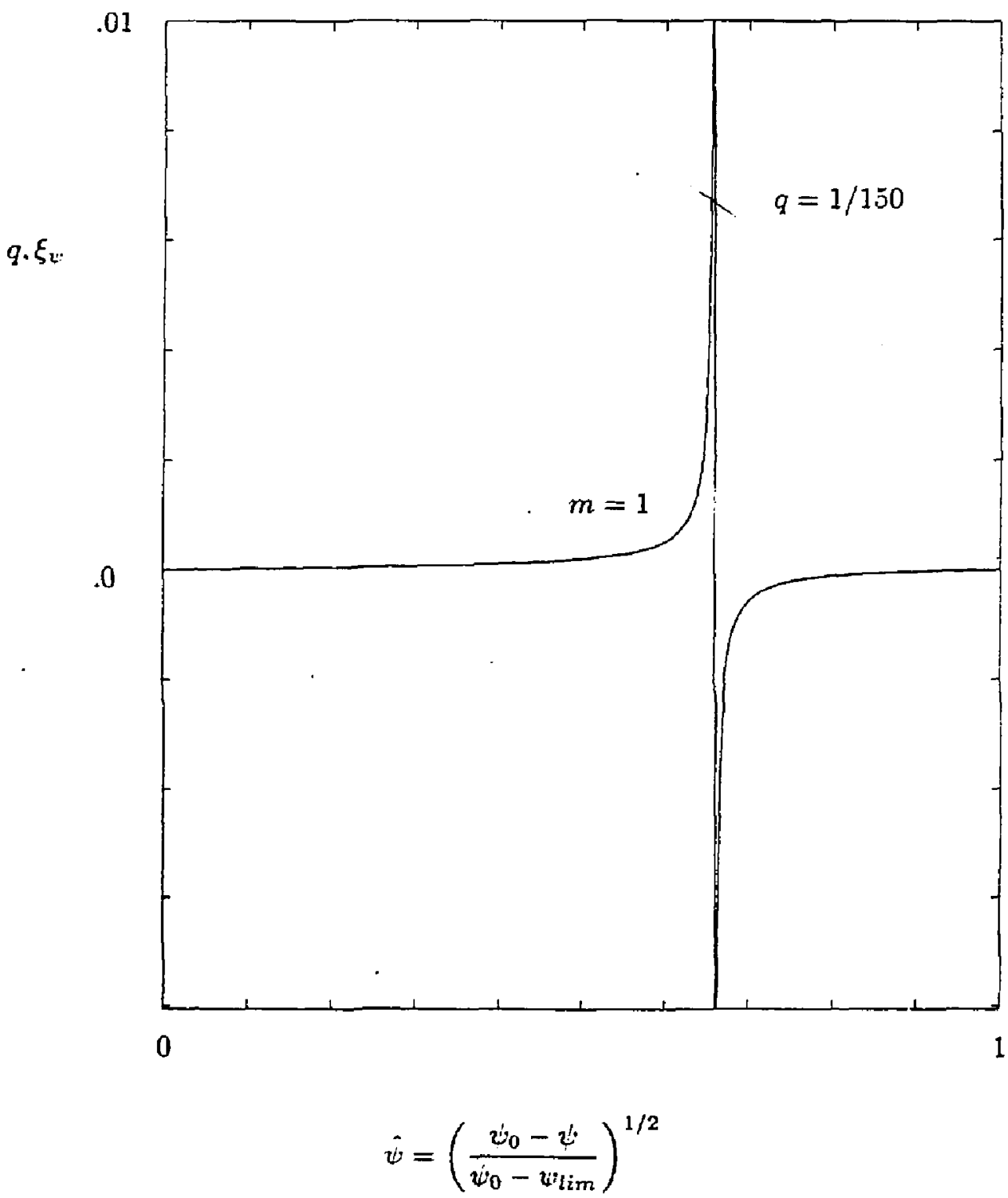

Fig. 22 
Figure 9 Scaling of Unstahle Mode
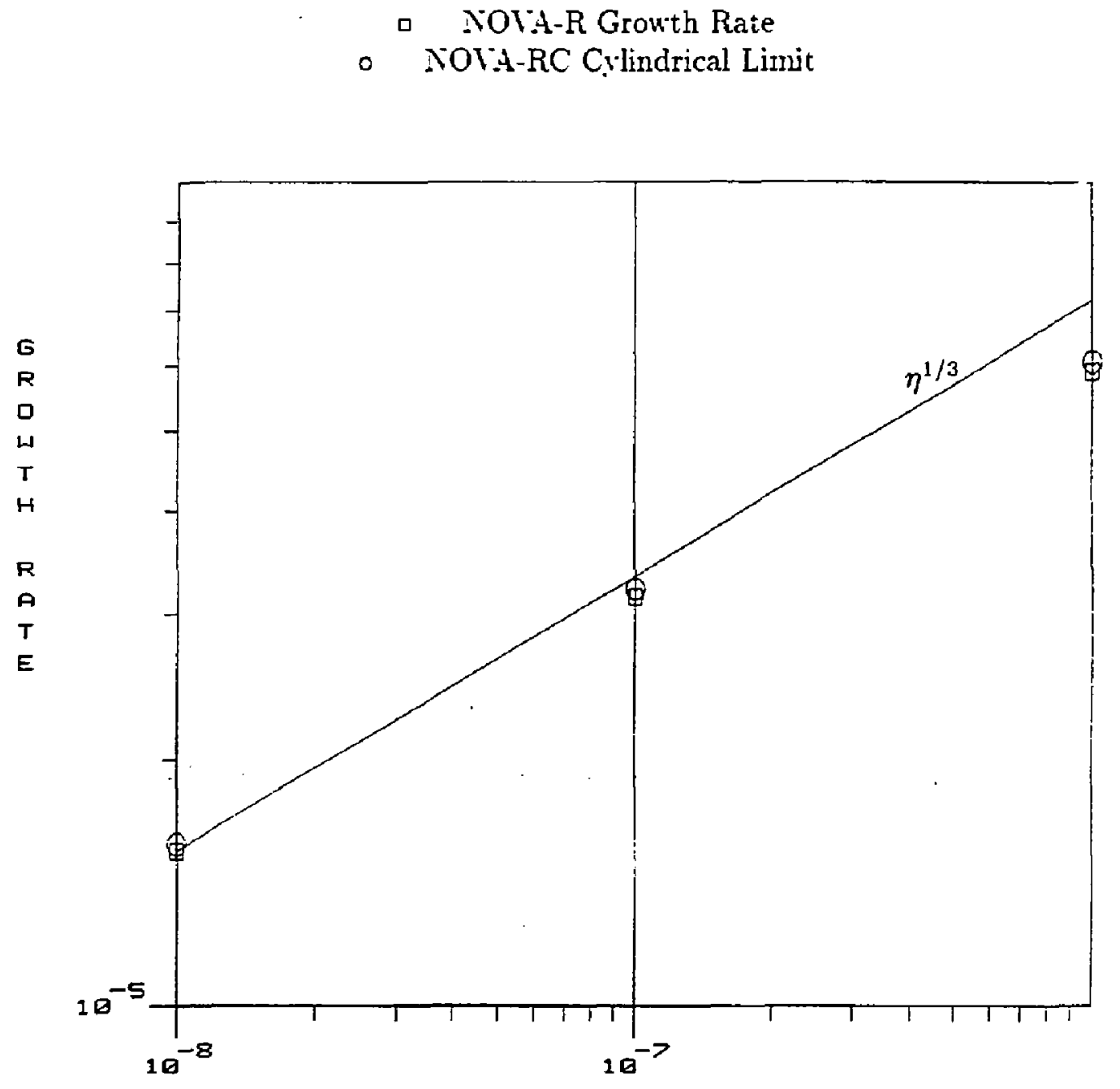

RESISTIUITY, INUERSE MAG REYNDLDS ND

F18. 23 
Figure 10 NOVA-R Tearing Mode

$$
\eta=10^{-7}
$$

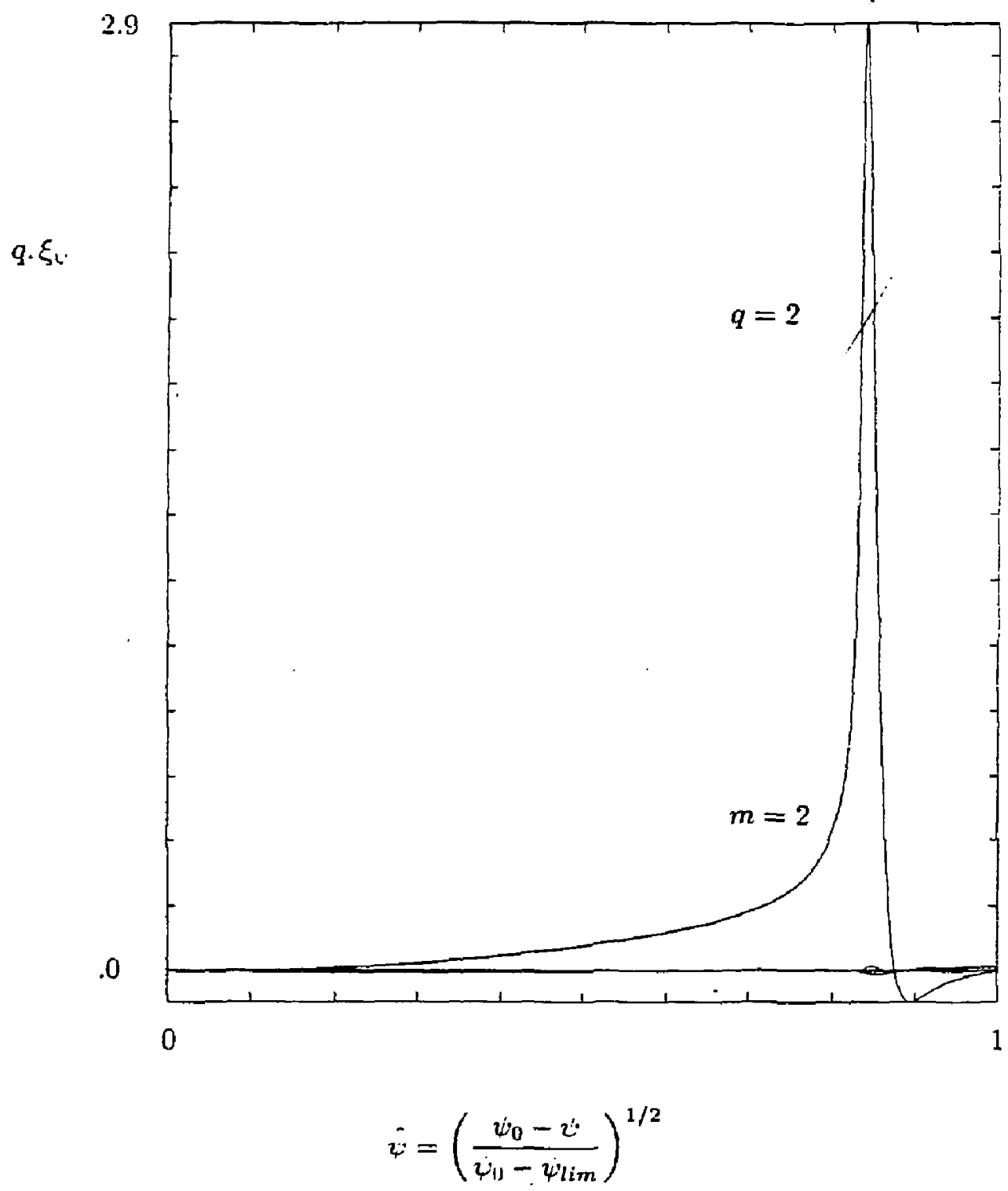

F1g. 24 
Figure 11 NOTA-R Tearing Mode

$$
\eta=10^{-10}
$$

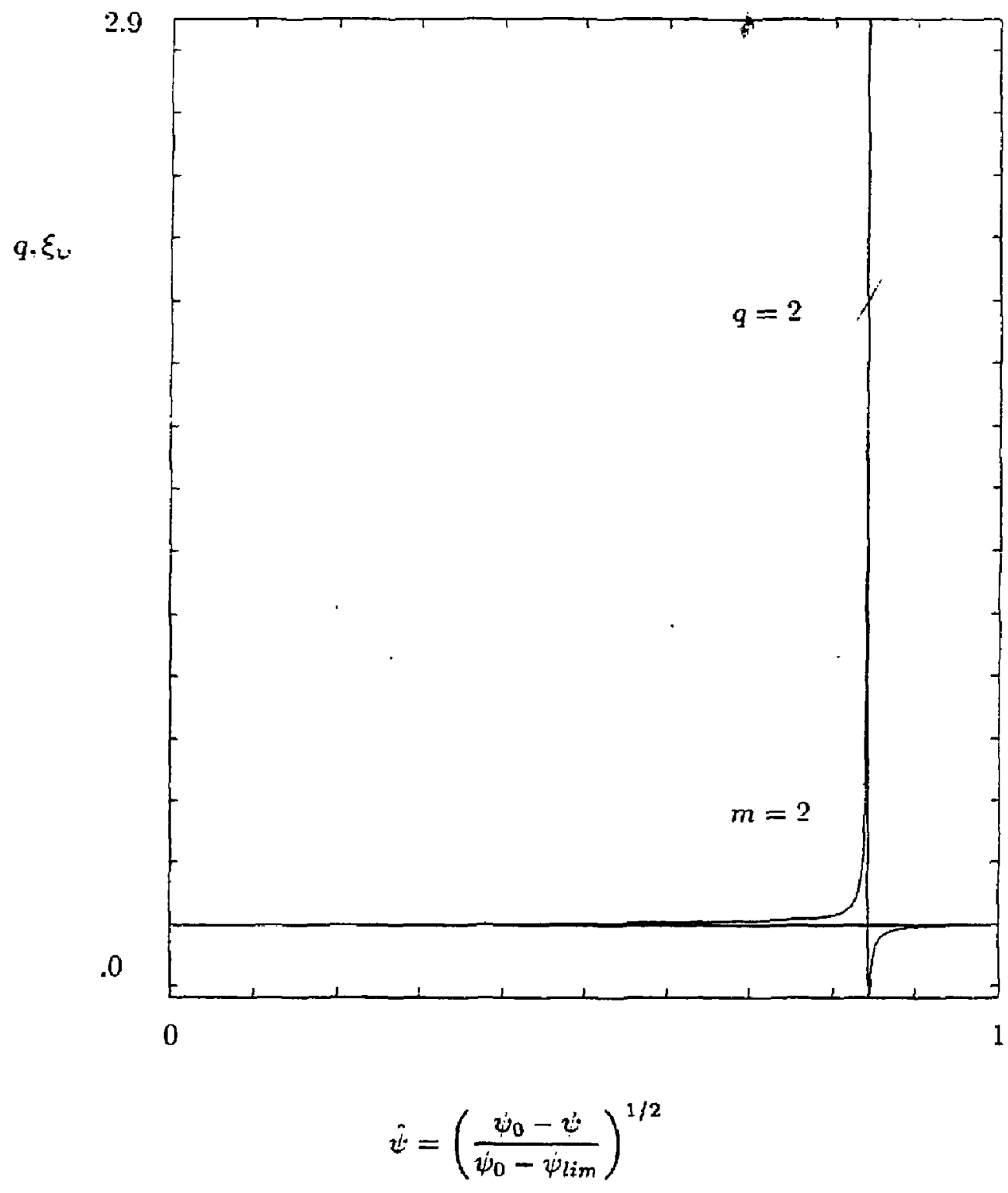

F1g. 25 
Figure 12a NOTA-R Tearing Mode Blowup

$$
\eta=10^{-10}
$$

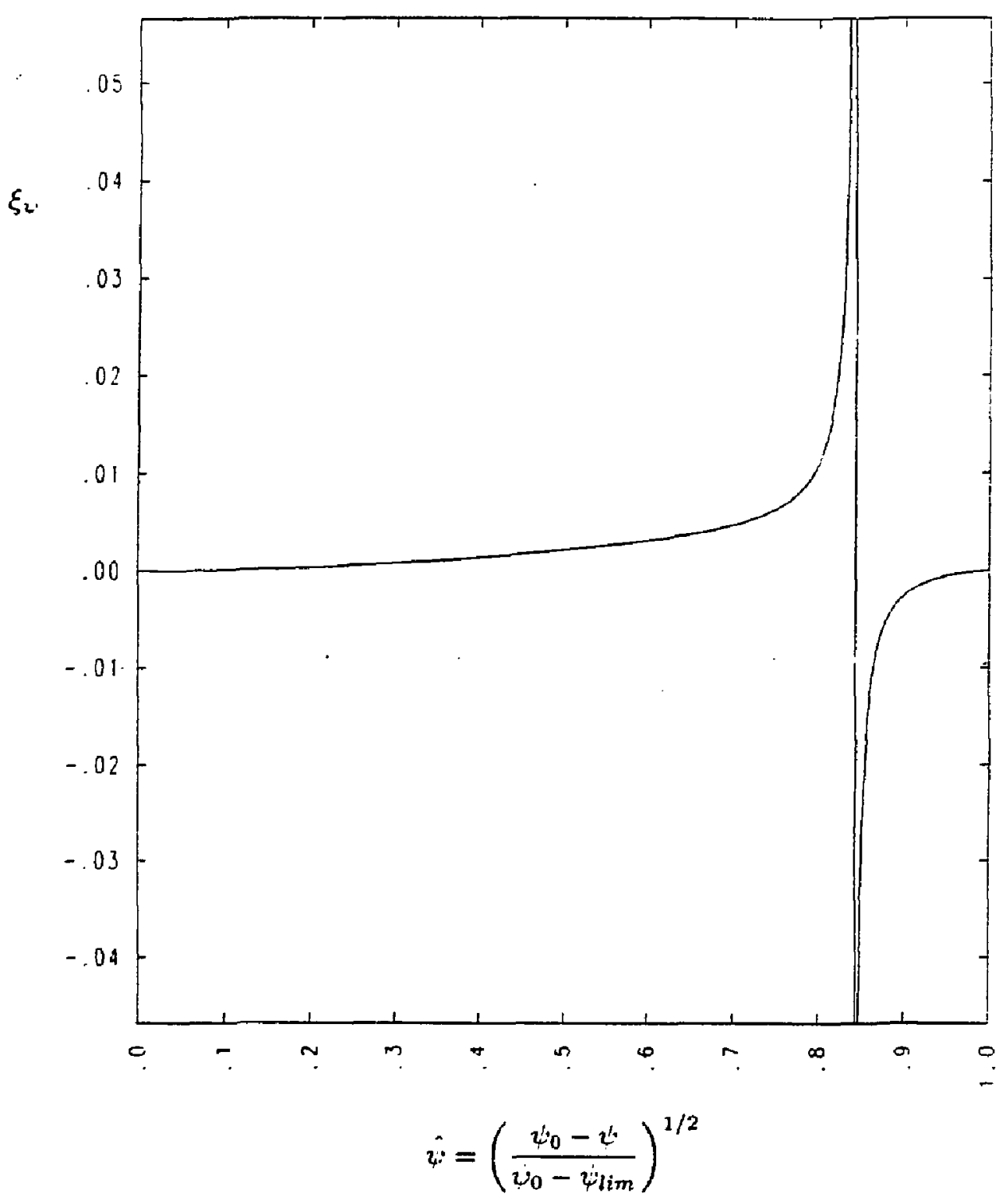

F1g. 26 
Figure 12b PESI Solution

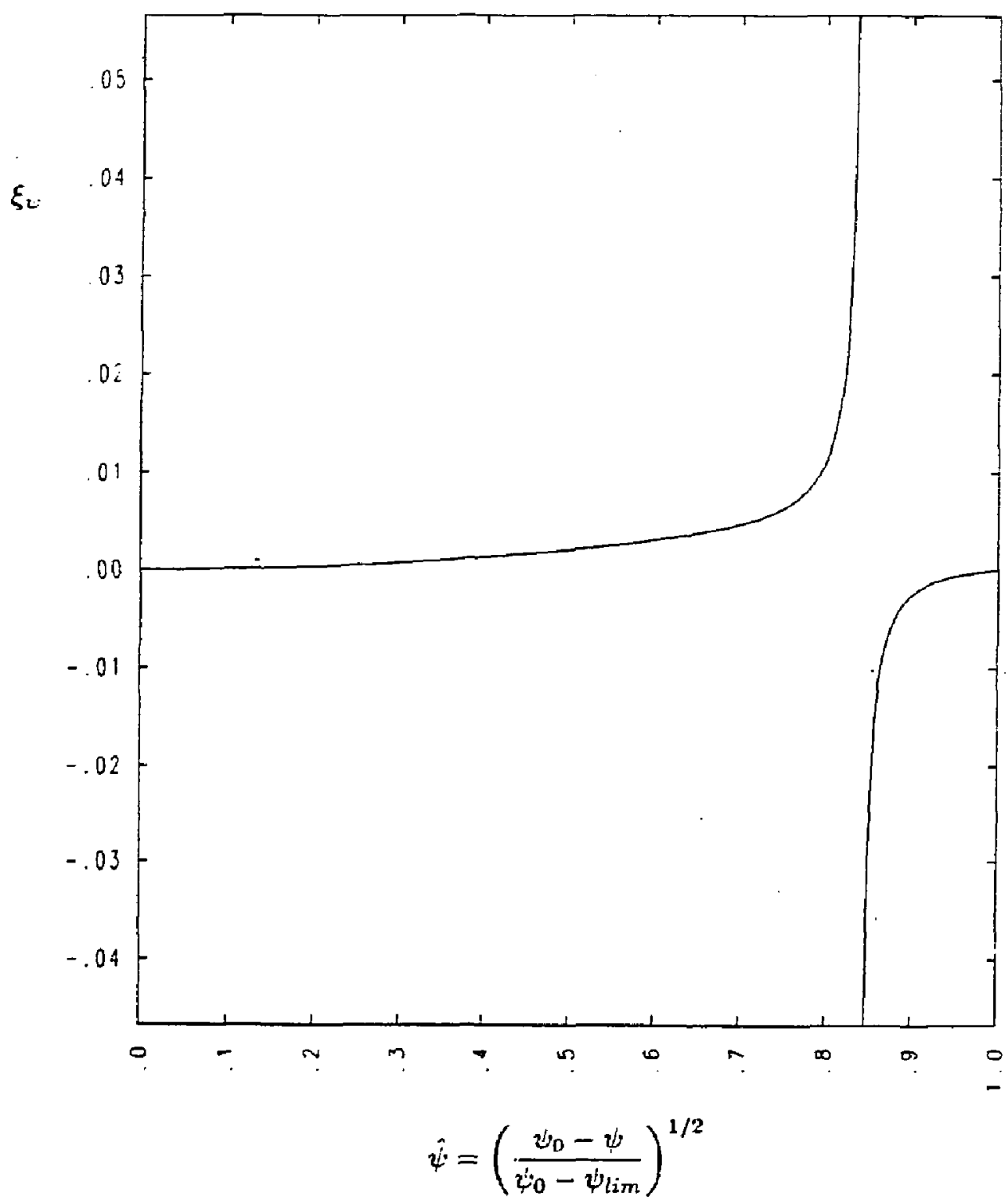

F1B, 27 
Figure 12c Superposition of NOVA R and PEST Tearing Mode

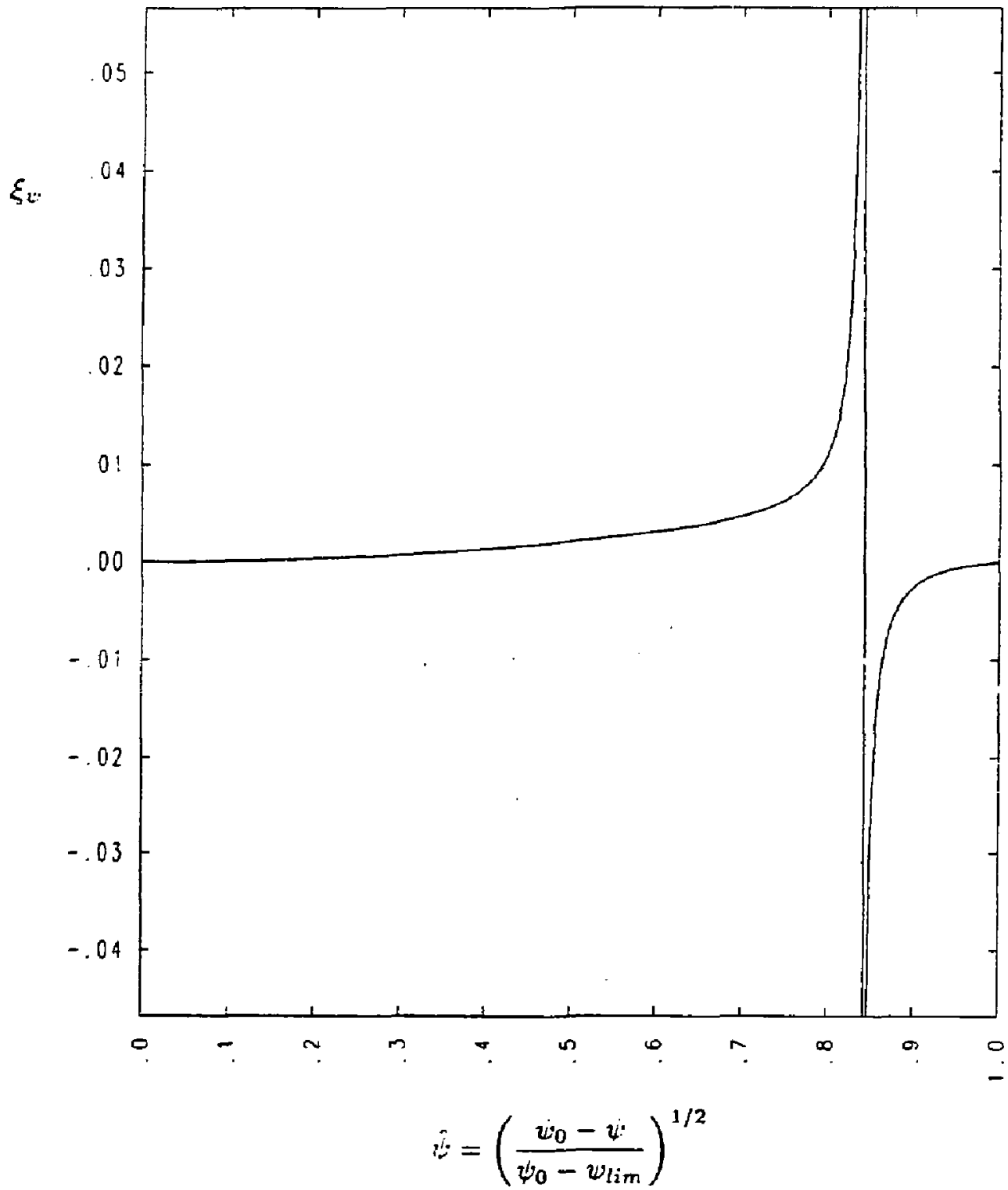

F1g. 28 
Figure 13 Scaling of Unstable Mode

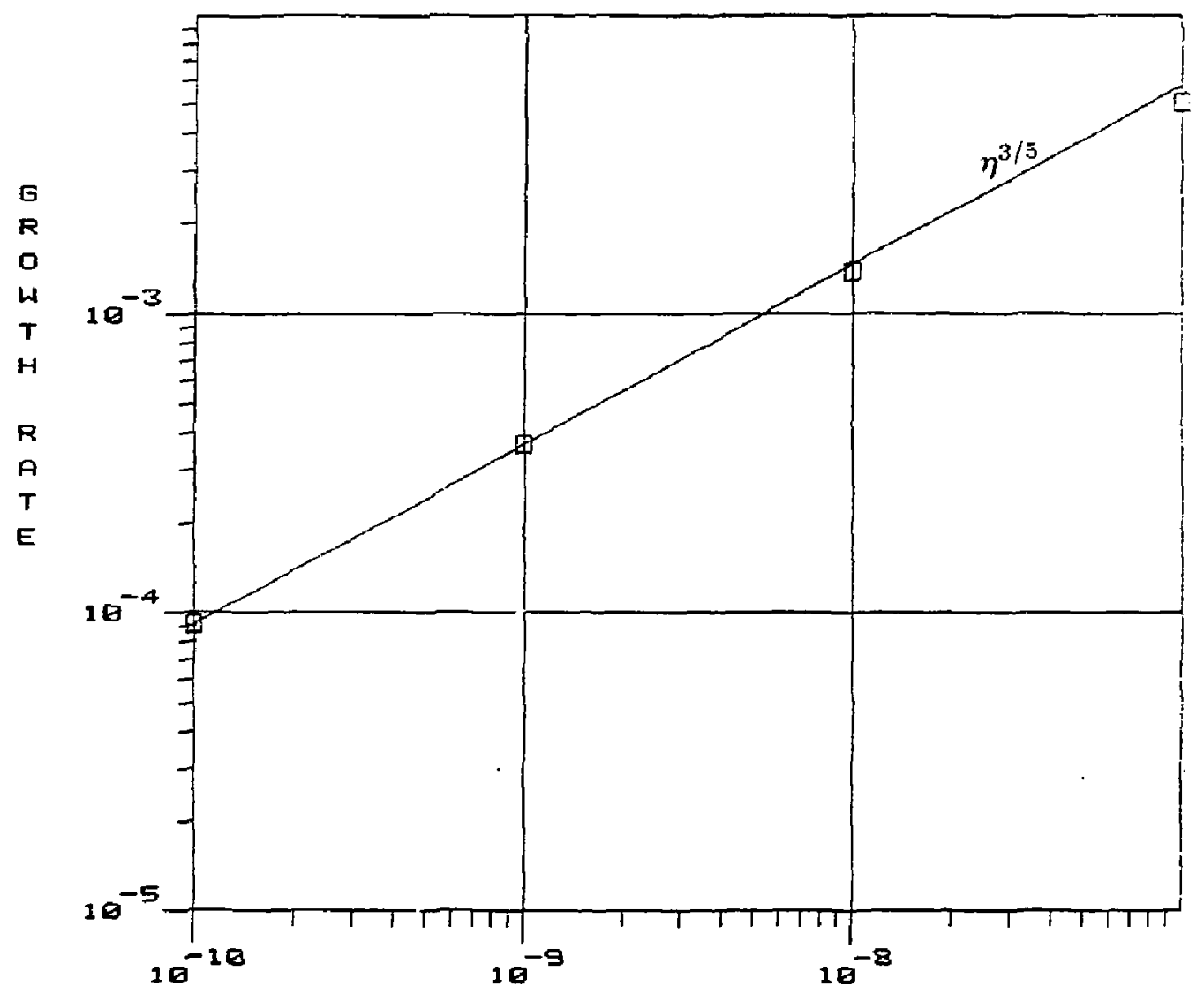

RESISTIUITY, INUERSE MAG REYNDLDS NO, 10

Fig. 29 
Fig. 14 Errors from three different computer packages

\title{
ERR
}

\author{
$\Delta$ Linear Finite Elements
}

I Texas Cubic B-Spline Package

O NOVA-R Cubic B-Spline Package

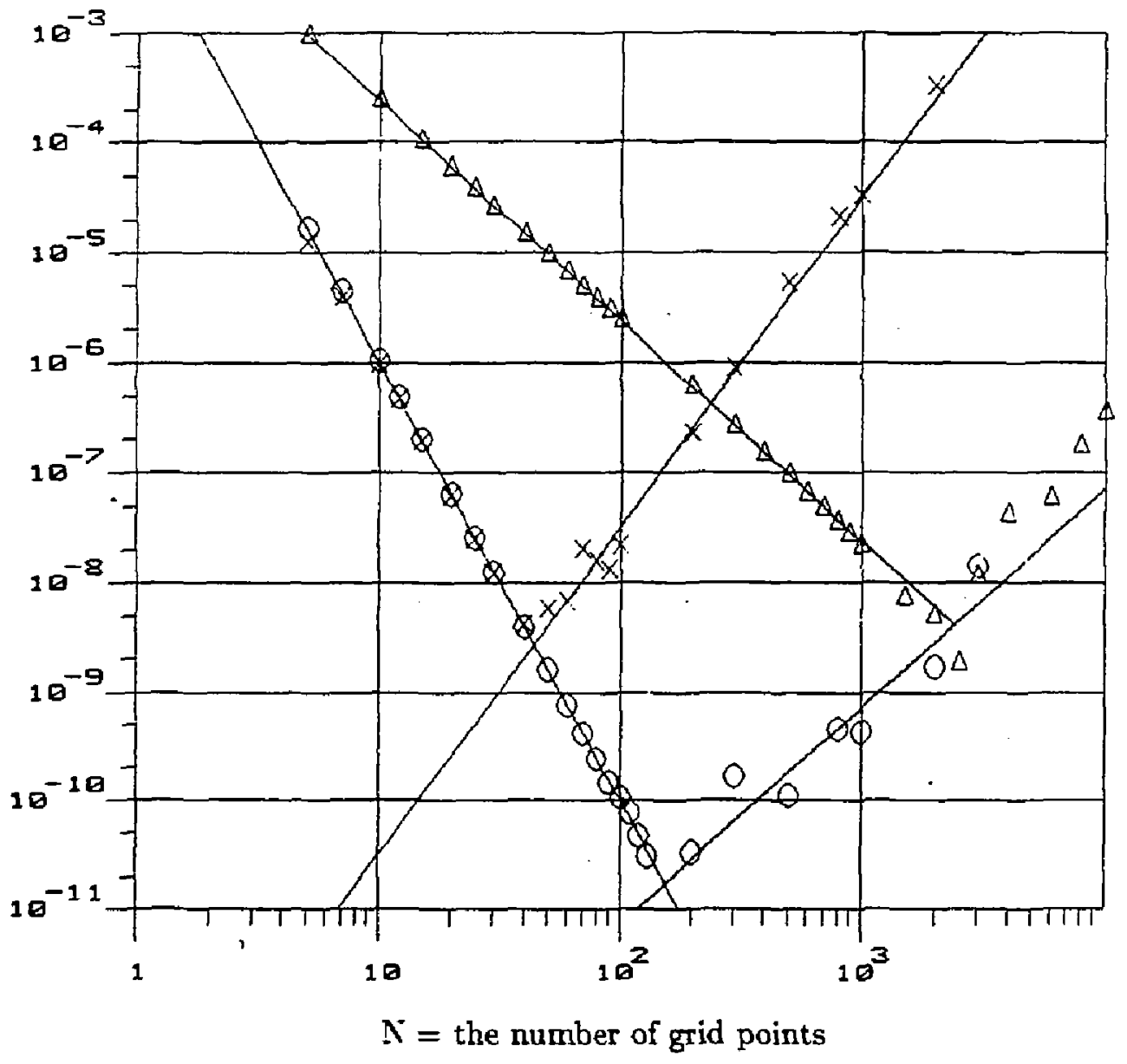

F1g. 30 